\title{
AN ERROR ANALYSIS OF DISCONTINUOUS FINITE ELEMENT METHODS FOR THE OPTIMAL CONTROL PROBLEMS GOVERNED BY STOKES EQUATION
}

\author{
ASHA K DOND, THIRUPATHI GUDI, AND RAMESH CH. SAU
}

\begin{abstract}
In this paper, an abstract framework for the error analysis of discontinuous finite element method is developed for the distributed and Neumann boundary control problems governed by the stationary Stokes equation with control constraints. A priori error estimates of optimal order are derived for velocity and pressure in the energy norm and the $L^{2}$-norm, respectively. Moreover, a reliable and efficient a posteriori error estimator is derived. The results are applicable to a variety of problems just under the minimal regularity possessed by the well-posedness of the problem. In particular, we consider the abstract results with suitable stable pairs of velocity and pressure spaces like as the lowestorder Crouzeix-Raviart finite element and piecewise constant spaces, piecewise linear and constant finite element spaces. The theoretical results are illustrated by the numerical experiments.
\end{abstract}

\section{INTRODUCTION}

We consider the following distributed control and Neumann boundary control problems governed by Stokes equations

$$
\min J(\mathbf{v}, \mathbf{x})=\frac{1}{2}\left\|\mathbf{v}-\mathbf{u}_{d}\right\|_{0, \Omega}^{2}+\frac{\lambda}{2}\|\mathbf{x}\|_{A}^{2},
$$

where $\|\cdot\|_{A}=\|\cdot\|_{0, \Omega}$ for distributed control problem and $\|\cdot\|_{A}=\|\cdot\|_{0, \partial \Omega}$ for Neumann boundary control problem, subject to,

For distributed control problem

$$
\begin{aligned}
-\Delta \mathbf{v}+\nabla q=\mathbf{x}+\mathbf{f} & \text { in } \Omega, \\
\nabla \cdot \mathbf{v} & =0 \quad \text { in } \Omega, \\
\mathbf{v} & =\mathbf{0} \quad \text { on } \partial \Omega, \\
\mathbf{y}_{a} \leq \mathbf{x}(x) \leq \mathbf{y}_{b} & \text { a.e. } x \in \Omega .
\end{aligned}
$$

For Neumann boundary control problem

$$
\begin{aligned}
&-\Delta \mathbf{v}+\nabla q=\mathbf{f} \text { in } \Omega, \\
& \nabla \cdot \mathbf{v}=0 \text { in } \Omega, \\
& \partial \mathbf{v} / \partial \mathbf{n}-q \mathbf{n}=\mathbf{x} \text { on } \partial \Omega, \\
& \mathbf{y}_{a} \leq \mathbf{x}(x) \leq \mathbf{y}_{b} \text { a.e. } x \in \partial \Omega .
\end{aligned}
$$

This paper investigates the discretization of the above systems based on a finite element approximation of the state and the control variable and also develop an abstract framework for the error analysis of the above problem under minimal regularity. The discussion of discretizations of optimal control problems governed by partial differential equations started

2020 Mathematics Subject Classification. 65N30; 65N15; 65N12; 65K10.

Key words and phrases. PDE-constrained optimization; Control-constraints; Finite element method; Discontinuous Galerkin method; Error bounds; Stokes equation. 
with papers of Falk [17], Gevici [18]. Subsequently, there are many significant contributions to this field. It is difficult to list all the results in this introduction; we refer to some of the articles and references therein for the development of numerical methods and their error analysis. Refer to the monograph [34] for the theory of optimal control problems and the development of numerical methods. The primal-dual active set algorithm has been developed in [24], and also it has been discussed in the context of the optimal control problems. Apart from this, we refer to [29] for a super-convergence result using a post-processed control for constrained control problems. A variational discretization method has been introduced in [25] to derive optimal error estimates by exploiting the relationship between the control and the adjoint state. For the numerical approximation of Neumann boundary control problem with graded mesh refinement refer to [1] and for the numerical treatment of the Dirichlet boundary control problems refer to [10, 13, 22, 28, 32] and references therein. On the other hand, while the adaptive finite element methods based on a posteriori error estimators have grown in popularity, the study of a posteriori error analysis for optimal control problems has also gained much interest in the recent years. In particular, the control in control constrained problem can exhibit kinks and hence lacks smoothness. In this context, adaptive finite element methods would be useful to enhance accuracy. An a posteriori error analysis of a conforming finite element method for control constrained problems has been derived in [23]. Recently, a general framework for a priori and a posteriori energy norm error analysis for Neumann and distributed control problems by discontinuous Galerkin discretization can be found in [11] for scalar problems. The results therein are obtained by the help of appropriate auxiliary problems. Local error analysis of discontinuous Galerkin methods for the distributed control problem for the advection-diffusion equation has been studied in [27].

Rösch and Vexler have applied the post-processing technique to a linear-quadratic optimal control problem governed by the Stokes equations [33]. They have proved second order convergence under the assumption that the velocity field admits full regularity, which means it is contained in $H^{2}(\Omega) \cap W^{1, \infty}(\Omega)$. Nicaise and Sirch [31] have extended the results of Rösch and Vexler [33] and Apel et al. [2, 3] to the conforming and nonconforming finite element methods for the optimal control of the Stokes equations under weaker regularity assumptions. This means, they did not assume that the velocity field is contained in $H^{2}(\Omega) \cap W^{1, \infty}(\Omega)$, but only in some weighted space $H_{\omega}^{2}(\Omega)$. The analysis in [31] is focused on the super-convergence result with regularity of the solution in some weighted Sobolev spaces. Our aim in this article is to derive the best approximation result in the energy norms under the weak regularity of the solution obtained through the weak formulation. It is natural to expect that the conforming methods exhibit this best approximation properties but it is not immediate for the nonconforming and discontinuous finite element methods. The nonconforming methods and discontinuous Galerkin methods are particularly attractive for the Stokes problem as they provide discrete inf-sup condition easily as compared to the conforming methods.

In this article, we consider a general optimality system of both the distributed and Neumann boundary control problem governed by Stokes equation. We develop an abstract 
framework for both a priori and a posteriori error analysis of the general optimality system under some abstract assumptions. We introduce two auxiliary problems: one dealing with an elliptic projection in a priori analysis and the other is based on a reconstruction in a posteriori error analysis. Subsequently, Theorem 2.3 and Theorem 2.7 are proved, which play an essential role in the analysis. In particular, we consider the abstract results with the lowest-order Crouzeix-Raviart finite element and piecewise constant spaces stable pair for velocity and pressure approximations respectively, and also discontinuous Galerkin formulation with piecewise linear and constant finite element spaces. The outcome of the result is the best approximation result for the numerical method. Furthermore, we derive the optimal order of convergence for control, state, and adjoint state variables. This framework for the error analysis of finite element methods for control problems have been presented under limited regularity assumptions. It is worth noting that the standard error analysis of DG methods require additional regularity which does not exist in several cases, for example in mixed boundary value problems or simply supported plates, example, see the discussions in [20]. Therefore, the error analysis of DG methods has to be treated carefully. Here, the best approximation error estimates are derived under the minimum regularity on the state and the adjoint state variables for DG methods. Moreover, a posteriori error estimators are derived for model problems, which are useful in adaptive mesh refinement algorithms. It is important to note that the best approximation results are key estimates in establishing the optimality of adaptive finite element methods. To the authors' best knowledge this is the first attempt of discussing the error analysis of DG methods under minimal regularity for the optimal control of the Stokes equations with pointwise control constraints.

This paper is organized as follows. Section 2 set up the abstract framework for the error analysis of discontinuous finite element methods and derives therein some abstract error estimates that form the basis for a priori. Subsection 2.2 deals with a posteriori error analysis. Section 3 introduces two model examples that are under discussion. In section 4 , we develop the discrete setting and discuss the applications to the model problems introduced in section 3. Section 5 presents some numerical examples to illustrate the theoretical results.

\section{Abstract Setting}

In this section, we develop an abstract framework for the error analysis of discontinuous and nonconforming finite element methods for approximating the solutions of optimal control problems with either boundary control or distributed control. We will assume all the vector spaces are real.

Let $X$ and $M$ are Hilbert spaces with the norm $\|\cdot\|_{X}$ and $\|\cdot\|_{M}$, respectively. We denote $V:=X \times M$ the admissible pair of spaces for state variables (velocity and pressure, respectively) and adjoint states. Let $V^{\prime}:=X^{\prime} \times M^{\prime}$, where $X^{\prime}$ and $M^{\prime}$ are dual of $X$ and $M$, respectively. Let $W$ be a Hilbert space such that $X \subset W \subset X^{\prime}$ and the inclusions are continuous. The inner product and the norm on $W$ are denoted by $\langle\cdot, \cdot\rangle_{W}$ and $\|\cdot\|_{W}$, respectively. Let $Q$ be a Hilbert space that will be used for seeking the control variable. The norm 
and inner product on $Q$ will be respectively denoted by $\|\cdot\|_{Q}$ and $\langle\cdot, \cdot\rangle_{Q}$. Let $E: X \rightarrow Q$ be a continuous linear operator. Let $Q_{a d} \subset Q$ be a nonempty closed convex subset.

Assume that $((\mathbf{u}, p),(\phi, r), \mathbf{y}) \in V \times V \times Q_{a d}$ solves the following optimality system

$$
\begin{aligned}
a(\mathbf{u}, \mathbf{z})+b(\mathbf{z}, p) & =\langle\mathbf{y}, E \mathbf{z}\rangle_{Q}+\langle\mathbf{f}, \mathbf{z}\rangle_{W} & & \text { for all } \mathbf{z} \in X, \\
b(\mathbf{u}, w) & =0 & & \text { for all } w \in M, \\
a(\mathbf{z}, \boldsymbol{\phi})-b(\mathbf{z}, r) & =\left\langle\mathbf{u}-\mathbf{u}_{d}, \mathbf{z}\right\rangle_{W} & & \text { for all } \mathbf{z} \in X, \\
b(\boldsymbol{\phi}, w) & =0 & & \text { for all } w \in M, \\
\langle E \boldsymbol{\phi}+\lambda \mathbf{y}, \mathbf{x}-\mathbf{y}\rangle_{Q} & \geq 0 & & \text { for all } \mathbf{x} \in Q_{a d},
\end{aligned}
$$

where $\mathbf{f} \in W, \mathbf{u}_{d} \in W, \lambda>0$ are given and $a: X \times X \rightarrow \mathbb{R}, b: X \times M \rightarrow \mathbb{R}$ are continuous bilinear forms in the sense that there exist $C_{1}, C_{2}>0$ such that

$$
|a(\mathbf{v}, \mathbf{z})| \leq C_{1}\|\mathbf{v}\|_{X}\|\mathbf{z}\|_{X}, \quad|b(\mathbf{z}, p)| \leq C_{2}\|\mathbf{z}\|_{X}\|p\|_{M},
$$

for all $\mathbf{v}, \mathbf{z} \in X$ and $p \in M$. The set $Z=\{\mathbf{v} \in X: \forall w \in M, b(\mathbf{v}, w)=0\}$ and $a$ is $Z$-elliptic, that is, there exists a constant $\alpha>0$ such that $\alpha\|\mathbf{v}\|_{X}^{2} \leq a(\mathbf{v}, \mathbf{v})$ for all $\mathbf{v} \in Z$, and $b$ satisfies the inf-sup condition which is given by

$$
\inf _{p \in M} \sup _{\mathbf{z} \in X} \frac{b(\mathbf{z}, p)}{\|\mathbf{z}\|_{X}\|p\|_{M}} \geq C, \text { for some } C>0 .
$$

Remark 2.1. We have assumed that the system (2.1) is well-posed and in Section 3, we have verified the well-posedness of (2.1) for two model problems.

Now we introduce corresponding discrete setting. Let $X_{h} \subset W$ be a finite dimensional subspace and $\|\cdot\|_{h}$ be a norm on $X+X_{h}$ such that $\|\mathbf{v}\|_{h}=\|\mathbf{v}\|_{X}$ for all $\mathbf{v} \in X$. Also let $M_{h}$ be a finite dimensional subspace of $M$ and the norm on $M_{h}$ is $\|\cdot\|_{M} \cdot a_{h}: X_{h} \times X_{h} \rightarrow \mathbb{R}$, $b_{h}: X_{h} \times M_{h} \rightarrow \mathbb{R}$ are continuous bilinear forms in the sense that there exist $\bar{C}_{1}, \bar{C}_{2}>0$ such that

$$
\left|a_{h}\left(\mathbf{v}_{h}, \mathbf{z}_{h}\right)\right| \leq \bar{C}_{1}\left\|\mathbf{v}_{h}\right\|_{h}\left\|\mathbf{z}_{h}\right\|_{h},\left|b_{h}\left(\mathbf{z}_{h}, p_{h}\right)\right| \leq \bar{C}_{2}\left\|\mathbf{z}_{h}\right\|_{h}\left\|p_{h}\right\|_{M}
$$

for all $\mathbf{v}_{h}, \mathbf{z}_{h} \in X_{h}$ and $p_{h} \in M_{h}$. The set

$$
Z_{h}=\left\{\mathbf{v}_{h} \in X_{h}: \forall w_{h} \in M_{h}, b_{h}\left(\mathbf{v}_{h}, w_{h}\right)=0\right\}
$$

and $a_{h}$ is $Z_{h}$-elliptic, that is, there exists $\bar{\alpha}>0$ independent of mesh-size such that $\bar{\alpha}\left\|\mathbf{v}_{h}\right\|_{h}^{2} \leq$ $a_{h}\left(\mathbf{v}_{h}, \mathbf{v}_{h}\right)$ for all $\mathbf{v}_{h} \in Z_{h}$ and $b_{h}$ satisfies the inf-sup condition

$$
\inf _{p_{h} \in M_{h}} \sup _{\mathbf{z}_{h} \in X_{h}} \frac{b_{h}\left(\mathbf{z}_{h}, p_{h}\right)}{\left\|\mathbf{z}_{h}\right\|_{h}\left\|p_{h}\right\|_{M}} \geq \bar{C}, \text { for some } \bar{C}>0
$$

which is independent of mesh-size. Similarly, assume that $Q_{h} \subseteq Q$ is a finite dimensional subspace and $Q_{a d}^{h} \subset Q_{a d}$ is nonempty closed convex subset of $Q_{h}$. We denote $V_{h}:=X_{h} \times M_{h}$.

Assume that $\left(\left(\mathbf{u}_{h}, p_{h}\right),\left(\boldsymbol{\phi}_{h}, r_{h}\right), \mathbf{y}_{h}\right) \in V_{h} \times V_{h} \times Q_{a d}^{h}$ solves the following optimality system:

$$
a_{h}\left(\mathbf{u}_{h}, \mathbf{z}_{h}\right)+b_{h}\left(\mathbf{z}_{h}, p_{h}\right)=\left\langle\mathbf{y}_{h}, E_{h} \mathbf{z}_{h}\right\rangle_{Q}+\left\langle\mathbf{f}, \mathbf{z}_{h}\right\rangle_{W} \quad \text { for all } \mathbf{z}_{h} \in X_{h},
$$




$$
\begin{aligned}
b_{h}\left(\mathbf{u}_{h}, w_{h}\right) & =0 & & \text { for all } w_{h} \in M_{h}, \\
a_{h}\left(\mathbf{z}_{h}, \phi_{h}\right)-b_{h}\left(\mathbf{z}_{h}, r_{h}\right) & =\left\langle\mathbf{u}_{h}-\mathbf{u}_{d}, \mathbf{z}_{h}\right\rangle_{W} & & \text { for all } \mathbf{z}_{h} \in X_{h}, \\
b_{h}\left(\boldsymbol{\phi}_{h}, w_{h}\right) & =0 & & \text { for all } w_{h} \in M_{h}, \\
\left\langle E_{h} \boldsymbol{\phi}_{h}+\lambda \mathbf{y}_{h}, \mathbf{x}_{h}-\mathbf{y}_{h}\right\rangle_{Q} & \geq 0 & & \text { for all } \mathbf{x}_{h} \in Q_{a d}^{h},
\end{aligned}
$$

where $E_{h}: X_{h}+X \rightarrow Q$ is a discrete counterpart of $E$ such that $E_{h} \mathbf{v}=E \mathbf{v}$ for all $\mathbf{v} \in X$. Throughout this section, we assume that the following hold true:

Assumption I: For all $\mathbf{v} \in X+X_{h}$,

$$
\|\mathbf{v}\|_{W} \leq C\|\mathbf{v}\|_{h}
$$

where $C$ is independent of mesh-size.

Assumption II: For all $\mathbf{v} \in X$, and $\mathbf{v}_{h} \in X_{h}$,

$$
\left\|E_{h}\left(\mathbf{v}-\mathbf{v}_{h}\right)\right\|_{Q} \leq\left\|\mathbf{v}-\mathbf{v}_{h}\right\|_{h} .
$$

Assumption III: The $Q$-projection defined as: For given $\mathbf{x} \in Q$, let $\Pi_{h} \mathbf{x} \in Q_{h}$ be the solution of

$$
\left\langle\Pi_{h} \mathbf{x}-\mathbf{x}, \mathbf{x}_{h}\right\rangle_{Q}=0 \text { for all } \mathbf{x}_{h} \in Q_{h} .
$$

Assume that $\Pi_{h} \mathbf{x} \in Q_{a d}^{h}$, whenever $\mathbf{x} \in Q_{a d}$.

2.1. A priori Error Analysis. To derive some abstract a priori error analysis, we introduce some projections as follows: Let $P_{h} \mathbf{u} \in X_{h}, \bar{P}_{h} \phi \in X_{h}, R_{h} p \in M_{h}$ and $\bar{R}_{h} r \in M_{h}$ solve

$$
\begin{aligned}
a_{h}\left(P_{h} \mathbf{u}, \mathbf{z}_{h}\right)+b_{h}\left(\mathbf{z}_{h}, R_{h} p\right) & =\left\langle\mathbf{y}, E_{h} \mathbf{z}_{h}\right\rangle_{Q}+\left\langle\mathbf{f}, \mathbf{z}_{h}\right\rangle_{W} & & \text { for all } \mathbf{z}_{h} \in X_{h}, \\
b_{h}\left(P_{h} \mathbf{u}, w_{h}\right) & =0 & & \text { for all } w_{h} \in M_{h}, \\
a_{h}\left(\mathbf{z}_{h}, \bar{P}_{h} \boldsymbol{\phi}\right)-b_{h}\left(\mathbf{z}_{h}, \bar{R}_{h} r\right) & =\left\langle\mathbf{u}-\mathbf{u}_{d}, \mathbf{z}_{h}\right\rangle_{W} & & \text { for all } \mathbf{z}_{h} \in X_{h}, \\
b_{h}\left(\bar{P}_{h} \boldsymbol{\phi}, w_{h}\right) & =0 & & \text { for all } w_{h} \in M_{h} .
\end{aligned}
$$

Here, we assumed that the bilinear forms $a_{h}$ and $b_{h}$ are continuous, $a_{h}$ is $Z_{h}$-elliptic, and $b_{h}$ is inf-sup stable. Also, the right-hand side of (2.7a) is a bounded linear functional on $X_{h}$. Hence the system (2.7a)-(2.7b) has a unique solution [19, pp. 112]. Similarly, the system $(2.7 \mathrm{c})-(2.7 \mathrm{~d})$ is well-posed.

To derive a priori error estimate of control, we need the following lemma.

Lemma 2.2. For all $\mathbf{x}_{h} \in Q_{a d}^{h}$, it holds

$\left\langle E_{h}\left(\boldsymbol{\phi}_{h}-\bar{P}_{h} \boldsymbol{\phi}\right), \mathbf{y}-\mathbf{y}_{h}\right\rangle_{Q} \geq\left\langle E_{h}\left(\boldsymbol{\phi}-\bar{P}_{h} \boldsymbol{\phi}\right), \mathbf{y}-\mathbf{y}_{h}\right\rangle_{Q}+\left\langle E_{h} \boldsymbol{\phi}_{h}+\lambda \mathbf{y}_{h}, \mathbf{y}-\mathbf{x}_{h}\right\rangle_{Q}+\lambda\left\|\mathbf{y}-\mathbf{y}_{h}\right\|_{Q}^{2}$.

Proof. For all $\mathbf{x}_{h} \in Q_{a d}^{h}$, we have

$$
\left\langle E_{h} \phi_{h}+\lambda \mathbf{y}_{h}, \mathbf{y}-\mathbf{y}_{h}\right\rangle_{Q}=\left\langle E_{h} \phi_{h}+\lambda \mathbf{y}_{h}, \mathbf{y}-\mathbf{x}_{h}\right\rangle_{Q}+\left\langle E_{h} \phi_{h}+\lambda \mathbf{y}_{h}, \mathbf{x}_{h}-\mathbf{y}_{h}\right\rangle_{Q} .
$$


From (2.3e),

$$
\left\langle E_{h} \boldsymbol{\phi}_{h}+\lambda \mathbf{y}_{h}, \mathbf{y}-\mathbf{y}_{h}\right\rangle_{Q} \geq\left\langle E_{h} \boldsymbol{\phi}_{h}+\lambda \mathbf{y}_{h}, \mathbf{y}-\mathbf{x}_{h}\right\rangle_{Q} .
$$

The substitution $\mathbf{x}=\mathbf{y}_{h} \in Q_{a d}^{h}\left(\subseteq Q_{a d}\right)$ and $E_{h} \phi=E \phi$ for $\phi \in X$ in (2.1e), imply

$$
-\left\langle E_{h} \boldsymbol{\phi}+\lambda \mathbf{y}, \mathbf{y}-\mathbf{y}_{h}\right\rangle_{Q} \geq 0 \text {. }
$$

An addition of (2.9) and (2.10), yields

$$
\left\langle E_{h}\left(\phi_{h}-\boldsymbol{\phi}\right)+\lambda\left(\mathbf{y}_{h}-\mathbf{y}\right), \mathbf{y}-\mathbf{y}_{h}\right\rangle_{Q} \geq\left\langle E_{h} \phi_{h}+\lambda \mathbf{y}_{h}, \mathbf{y}-\mathbf{x}_{h}\right\rangle_{Q}
$$

Hence,

$\left\langle E_{h}\left(\boldsymbol{\phi}_{h}-\bar{P}_{h} \boldsymbol{\phi}\right), \mathbf{y}-\mathbf{y}_{h}\right\rangle_{Q} \geq \lambda\left\|\mathbf{y}-\mathbf{y}_{h}\right\|_{Q}^{2}+\left\langle E_{h}\left(\boldsymbol{\phi}-\bar{P}_{h} \boldsymbol{\phi}\right), \mathbf{y}-\mathbf{y}_{h}\right\rangle_{Q}+\left\langle E_{h} \boldsymbol{\phi}_{h}+\lambda \mathbf{y}_{h}, \mathbf{y}-\mathbf{x}_{h}\right\rangle_{Q}$.

This concludes the proof

Following theorem gives an a priori error estimate for the control variable.

Theorem 2.3. Let $((\mathbf{u}, p),(\boldsymbol{\phi}, r), \mathbf{y})$ and $\left(\left(\mathbf{u}_{h}, p_{h}\right),\left(\phi_{h}, r_{h}\right), \mathbf{y}_{h}\right)$ solve systems (2.1), and (2.3), respectively. Then it holds

$\left\|\mathbf{y}-\mathbf{y}_{h}\right\|_{Q}^{2}+\left\|\mathbf{u}-\mathbf{u}_{h}\right\|_{W}^{2} \leq C\left(\left\|E \boldsymbol{\phi}-\Pi_{h}(E \boldsymbol{\phi})\right\|_{Q}^{2}+\left\|\mathbf{y}-\Pi_{h} \mathbf{y}\right\|_{Q}^{2}+\left\|\boldsymbol{\phi}-\bar{P}_{h} \boldsymbol{\phi}\right\|_{h}^{2}+\left\|\mathbf{u}-P_{h} \mathbf{u}\right\|_{W}^{2}\right)$.

Proof. From (2.7a) $-(2.3 \mathrm{a})$ and (2.7b)-(2.3b) for all $\left(\mathbf{z}_{h}, w_{h}\right) \in X_{h} \times M_{h}$ we have

$$
\begin{aligned}
a_{h}\left(P_{h} \mathbf{u}-\mathbf{u}_{h}, \mathbf{z}_{h}\right)+b_{h}\left(\mathbf{z}_{h}, R_{h} p-p_{h}\right) & =\left\langle\mathbf{y}-\mathbf{y}_{h}, E_{h} \mathbf{z}_{h}\right\rangle_{Q}, \\
b_{h}\left(P_{h} \mathbf{u}-\mathbf{u}_{h}, w_{h}\right) & =0 .
\end{aligned}
$$

Similarly, from (2.7c)-(2.3c) and (2.7d) $-(2.3 \mathrm{~d})$ for all $\left(\mathbf{z}_{h}, w_{h}\right) \in X_{h} \times M_{h}$, we have

$$
\begin{aligned}
a_{h}\left(\mathbf{z}_{h}, \bar{P}_{h} \boldsymbol{\phi}-\boldsymbol{\phi}_{h}\right)+b_{h}\left(\mathbf{z}_{h},-\bar{R}_{h} r+r_{h}\right) & =\left\langle\mathbf{u}-\mathbf{u}_{h}, \mathbf{z}_{h}\right\rangle_{W}, \\
b_{h}\left(\bar{P}_{h} \boldsymbol{\phi}-\boldsymbol{\phi}_{h}, w_{h}\right) & =0 .
\end{aligned}
$$

The substitution of $\mathbf{z}_{h}=\bar{P}_{h} \boldsymbol{\phi}-\boldsymbol{\phi}_{h}$ in (2.12) and $\mathbf{z}_{h}=P_{h} \mathbf{u}-\mathbf{u}_{h}$ in (2.14), and use of the fact that $b_{h}\left(P_{h} \mathbf{u}-\mathbf{u}_{h},-\bar{R}_{h} r+r_{h}\right)=0$ in (2.12) and $b_{h}\left(\bar{P}_{h} \boldsymbol{\phi}-\boldsymbol{\phi}_{h}, R_{h} p-p_{h}\right)=0$ in (2.14), and finally subtraction of the resulting equations lead to

$$
\left\langle\mathbf{y}-\mathbf{y}_{h}, E_{h}\left(\bar{P}_{h} \boldsymbol{\phi}-\boldsymbol{\phi}_{h}\right)\right\rangle_{Q}-\left\langle\mathbf{u}-\mathbf{u}_{h}, P_{h} \mathbf{u}-\mathbf{u}_{h}\right\rangle_{W}=0 .
$$

Further, we have

$$
\left\langle\mathbf{y}-\mathbf{y}_{h}, E_{h}\left(\phi_{h}-\bar{P}_{h} \phi\right)\right\rangle_{Q}+\left\|P_{h} \mathbf{u}-\mathbf{u}_{h}\right\|_{W}^{2}=\left\langle\mathbf{u}-P_{h} \mathbf{u}, \mathbf{u}_{h}-P_{h} \mathbf{u}\right\rangle_{W} .
$$

An addition of (2.16) and (2.8) from Lemma 2.2 with $\mathbf{x}_{h}=\Pi_{h} \mathbf{y}$ yields

$$
\lambda\left\|\mathbf{y}-\mathbf{y}_{h}\right\|_{Q}^{2}+\left\|P_{h} \mathbf{u}-\mathbf{u}_{h}\right\|_{W}^{2} \leq-\left\langle E_{h} \boldsymbol{\phi}_{h}+\lambda \mathbf{y}_{h}, \mathbf{y}-\mathbf{x}_{h}\right\rangle_{Q}
$$

$$
-\left\langle E_{h}\left(\boldsymbol{\phi}-\bar{P}_{h} \boldsymbol{\phi}\right), \mathbf{y}-\mathbf{y}_{h}\right\rangle_{Q}+\left\langle\mathbf{u}-P_{h} \mathbf{u}, \mathbf{u}_{h}-P_{h} \mathbf{u}\right\rangle_{W} .
$$


An addition and subtraction of some terms in the first term on the right-hand side of (2.17) shows

$$
\begin{aligned}
\left\langle E_{h} \boldsymbol{\phi}_{h}+\lambda \mathbf{y}_{h}, \mathbf{y}-\mathbf{x}_{h}\right\rangle_{Q}= & -\left\langle E_{h}\left(\phi_{h}-\boldsymbol{\phi}+\boldsymbol{\phi}\right)+\lambda\left(\mathbf{y}_{h}-\mathbf{y}+\mathbf{y}\right), \mathbf{y}-\mathbf{x}_{h}\right\rangle_{Q} \\
= & -\left\langle E_{h} \boldsymbol{\phi}-\Pi_{h}(E \boldsymbol{\phi})+\Pi_{h}(E \boldsymbol{\phi})+\lambda\left(\mathbf{y}-\mathbf{x}_{h}+\mathbf{x}_{h}\right), \mathbf{y}-\mathbf{x}_{h}\right\rangle_{Q} \\
& -\left\langle E_{h}\left(\boldsymbol{\phi}_{h}-\boldsymbol{\phi}\right)+\lambda\left(\mathbf{y}_{h}-\mathbf{y}\right), \mathbf{y}-\mathbf{x}_{h}\right\rangle_{Q} \\
= & -\left\langle E \boldsymbol{\phi}-\Pi_{h}(E \boldsymbol{\phi})+\lambda\left(\mathbf{y}-\mathbf{x}_{h}\right), \mathbf{y}-\mathbf{x}_{h}\right\rangle_{Q}-\left\langle\Pi_{h}(E \boldsymbol{\phi})+\lambda \mathbf{x}_{h}, \mathbf{y}-\mathbf{x}_{h}\right\rangle_{Q} \\
& -\left\langle E_{h}\left(\boldsymbol{\phi}_{h}-\boldsymbol{\phi}\right)+\lambda\left(\mathbf{y}_{h}-\mathbf{x}_{h}+\mathbf{x}_{h}-\mathbf{y}\right), \mathbf{y}-\mathbf{x}_{h}\right\rangle_{Q} \\
= & -\left\langle E \boldsymbol{\phi}-\Pi_{h}(E \boldsymbol{\phi})+\lambda\left(\mathbf{y}-\mathbf{x}_{h}\right), \mathbf{y}-\mathbf{x}_{h}\right\rangle_{Q} \\
& -\left\langle E_{h}\left(\boldsymbol{\phi}_{h}-\boldsymbol{\phi}\right)+\lambda\left(\mathbf{x}_{h}-\mathbf{y}\right), \mathbf{y}-\mathbf{x}_{h}\right\rangle_{Q} .
\end{aligned}
$$

Here, the selection of $\mathbf{x}_{h}=\Pi_{h} \mathbf{y}$ and the $Q$-orthogonal projection property imply

$$
\left\langle\Pi_{h}(E \boldsymbol{\phi})+\lambda \mathbf{x}_{h}, \mathbf{y}-\mathbf{x}_{h}\right\rangle_{Q}-\left\langle\lambda\left(\mathbf{y}_{h}-\mathbf{x}_{h}\right), \mathbf{y}-\mathbf{x}_{h}\right\rangle_{Q}=0 .
$$

The substitution of (2.18) in (2.17) and a use of the Young inequality imply

$$
\begin{aligned}
\lambda\left\|\mathbf{y}-\mathbf{y}_{h}\right\|_{Q}^{2}+\left\|P_{h} \mathbf{u}-\mathbf{u}_{h}\right\|_{W}^{2} \leq & \left(\frac{1}{2}\left\|E \boldsymbol{\phi}-\Pi_{h}(E \boldsymbol{\phi})\right\|_{Q}^{2}+\frac{1}{2}\left\|\mathbf{y}-\mathbf{x}_{h}\right\|_{Q}^{2}+\frac{1}{2 \lambda}\left\|E_{h}\left(\boldsymbol{\phi}-\bar{P}_{h} \boldsymbol{\phi}\right)\right\|_{Q}^{2}\right) \\
& +\left(\left\|E_{h}\left(\boldsymbol{\phi}_{h}-\boldsymbol{\phi}\right)\right\|_{Q}\left\|\mathbf{y}-\mathbf{x}_{h}\right\|_{Q}+\frac{1}{2}\left\|\mathbf{u}-P_{h} \mathbf{u}\right\|_{W}^{2}\right) \\
& +\frac{1}{2}\left\|\mathbf{u}_{h}-P_{h} \mathbf{u}\right\|_{W}^{2}+\frac{\lambda}{2}\left\|\mathbf{y}-\mathbf{y}_{h}\right\|_{Q}^{2} .
\end{aligned}
$$

In order to estimate $\left\|E_{h}\left(\phi_{h}-\phi\right)\right\|_{Q}$, the choice of $\mathbf{z}_{h}=\bar{P}_{h} \boldsymbol{\phi}-\boldsymbol{\phi}_{h}$ in (2.14) shows

$$
a_{h}\left(\bar{P}_{h} \boldsymbol{\phi}-\boldsymbol{\phi}_{h}, \bar{P}_{h} \boldsymbol{\phi}-\boldsymbol{\phi}_{h}\right)+b_{h}\left(\bar{P}_{h} \boldsymbol{\phi}-\boldsymbol{\phi}_{h},-\bar{R}_{h} r+r_{h}\right)=\left\langle\mathbf{u}-\mathbf{u}_{h}, \bar{P}_{h} \boldsymbol{\phi}-\boldsymbol{\phi}_{h}\right\rangle_{W} .
$$

Since $\bar{P}_{h} \phi-\phi_{h} \in Z_{h}$, the $Z_{h}$-ellipticity of $a_{h}$ yields

$$
C\left\|\bar{P}_{h} \boldsymbol{\phi}-\boldsymbol{\phi}_{h}\right\|_{h}^{2} \leq\left\|\mathbf{u}-\mathbf{u}_{h}\right\|_{W}\left\|\bar{P}_{h} \boldsymbol{\phi}-\phi_{h}\right\|_{W} .
$$

From Assumption I, we have $\left\|\bar{P}_{h} \boldsymbol{\phi}-\boldsymbol{\phi}_{h}\right\|_{W} \leq\left\|\bar{P}_{h} \boldsymbol{\phi}-\boldsymbol{\phi}_{h}\right\|_{h}$. Hence

$$
\left\|\bar{P}_{h} \boldsymbol{\phi}-\boldsymbol{\phi}_{h}\right\|_{h} \leq C\left\|\mathbf{u}-\mathbf{u}_{h}\right\|_{W} \text {. }
$$

Also, from Assumption II, we have

$$
\begin{aligned}
\left\|E_{h}\left(\boldsymbol{\phi}-\boldsymbol{\phi}_{h}\right)\right\|_{Q} & \leq C\left\|\boldsymbol{\phi}-\boldsymbol{\phi}_{h}\right\|_{h} \leq C\left(\left\|\boldsymbol{\phi}-\bar{P}_{h} \boldsymbol{\phi}\right\|_{h}+\left\|\bar{P}_{h} \boldsymbol{\phi}-\boldsymbol{\phi}_{h}\right\|_{h}\right) \\
& \leq C\left(\left\|\boldsymbol{\phi}-\bar{P}_{h} \boldsymbol{\phi}\right\|_{h}+\left\|\mathbf{u}-\mathbf{u}_{h}\right\|_{W}\right) \\
& \leq C\left(\left\|\boldsymbol{\phi}-\bar{P}_{h} \boldsymbol{\phi}\right\|_{h}+\left\|\mathbf{u}-P_{h} \mathbf{u}\right\|_{W}+\left\|P_{h} \mathbf{u}-\mathbf{u}_{h}\right\|_{W}\right) .
\end{aligned}
$$

A substitution of (2.21) in (2.19), then a use of the Young inequality with kick-back the term $\left\|P_{h} \mathbf{u}-\mathbf{u}_{h}\right\|_{W}^{2}$ (to LHS) and the orthogonality property of $Q$-projection from Assumption III in the estimates of $\left\|\mathbf{y}-\mathbf{x}_{h}\right\|_{Q}^{2}$ result in

$$
\begin{aligned}
\left\|\mathbf{y}-\mathbf{y}_{h}\right\|_{Q}^{2}+\left\|P_{h} \mathbf{u}-\mathbf{u}_{h}\right\|_{W}^{2} \leq & C\left(\left\|E \boldsymbol{\phi}-\Pi_{h}(E \boldsymbol{\phi})\right\|_{Q}^{2}+\left\|\mathbf{y}-\Pi_{h} \mathbf{y}\right\|_{Q}^{2}\right. \\
& \left.+\left\|\boldsymbol{\phi}-\bar{P}_{h} \boldsymbol{\phi}\right\|_{h}^{2}+\left\|\mathbf{u}-P_{h} \mathbf{u}\right\|_{W}^{2}\right) .
\end{aligned}
$$


Finally, the triangle inequality in the term $\left\|\mathbf{u}-\mathbf{u}_{h}\right\|_{W}$ and (2.22) lead to (2.11). This concludes the proof.

Theorem 2.4. It holds,

$$
\left\|\boldsymbol{\phi}-\boldsymbol{\phi}_{h}\right\|_{h} \leq C\left(\left\|\boldsymbol{\phi}-\bar{P}_{h} \boldsymbol{\phi}\right\|_{h}+\left\|E \boldsymbol{\phi}-\Pi_{h}(E \boldsymbol{\phi})\right\|_{Q}+\left\|\mathbf{y}-\Pi_{h} \mathbf{y}\right\|_{Q}+\left\|\mathbf{u}-P_{h} \mathbf{u}\right\|_{W}\right)
$$

and

$$
\left\|\mathbf{u}-\mathbf{u}_{h}\right\|_{h} \leq C\left(\left\|\mathbf{u}-P_{h} \mathbf{u}\right\|_{h}+\left\|\boldsymbol{\phi}-\bar{P}_{h} \boldsymbol{\phi}\right\|_{h}+\left\|E \boldsymbol{\phi}-\Pi_{h}(E \boldsymbol{\phi})\right\|_{Q}+\left\|\mathbf{y}-\Pi_{h} \mathbf{y}\right\|_{Q}\right) .
$$

Proof. From (2.20) and (2.11), we have

$$
\begin{aligned}
\left\|\boldsymbol{\phi}-\boldsymbol{\phi}_{h}\right\|_{h} & \leq\left\|\boldsymbol{\phi}-\bar{P}_{h} \boldsymbol{\phi}\right\|_{h}+\left\|\bar{P}_{h} \boldsymbol{\phi}-\boldsymbol{\phi}_{h}\right\|_{h} \leq\left\|\boldsymbol{\phi}-\bar{P}_{h} \boldsymbol{\phi}\right\|_{h}+C\left\|\mathbf{u}-\mathbf{u}_{h}\right\|_{W} \\
& \leq C\left(\left\|\boldsymbol{\phi}-\bar{P}_{h} \boldsymbol{\phi}\right\|_{h}+\left\|E \boldsymbol{\phi}-\Pi_{h}(E \boldsymbol{\phi})\right\|_{Q}+\left\|\mathbf{y}-\Pi_{h} \mathbf{y}\right\|_{Q}+\left\|\mathbf{u}-P_{h} \mathbf{u}\right\|_{W}\right) .
\end{aligned}
$$

For the estimate of $\left\|\mathbf{u}-\mathbf{u}_{h}\right\|_{h}$, the substitution $\mathbf{z}_{h}=P_{h} \mathbf{u}-\mathbf{u}_{h}$ in (2.12) and use of (2.13) imply

$$
a_{h}\left(P_{h} \mathbf{u}-\mathbf{u}_{h}, P_{h} \mathbf{u}-\mathbf{u}_{h}\right)=\left\langle\mathbf{y}-\mathbf{y}_{h}, E_{h}\left(P_{h} \mathbf{u}-\mathbf{u}_{h}\right)\right\rangle_{Q}
$$

The $Z_{h}$-ellipticity and Assumption II give $\left\|P_{h} \mathbf{u}-\mathbf{u}_{h}\right\|_{h} \leq C\left\|\mathbf{y}-\mathbf{y}_{h}\right\|_{Q}$. Hence

$$
\begin{aligned}
\left\|\mathbf{u}-\mathbf{u}_{h}\right\|_{h} & \leq\left\|\mathbf{u}-P_{h} \mathbf{u}\right\|_{h}+\left\|P_{h} \mathbf{u}-\mathbf{u}_{h}\right\|_{h}, \leq\left\|\mathbf{u}-P_{h} \mathbf{u}\right\|_{h}+C\left\|\mathbf{y}-\mathbf{y}_{h}\right\|_{Q} \\
& \leq C\left(\left\|\mathbf{u}-P_{h} \mathbf{u}\right\|_{h}+\left\|\boldsymbol{\phi}-\bar{P}_{h} \boldsymbol{\phi}\right\|_{h}+\left\|E \boldsymbol{\phi}-\Pi_{h}(E \boldsymbol{\phi})\right\|_{Q}+\left\|\mathbf{y}-\Pi_{h} \mathbf{y}\right\|_{Q}\right)
\end{aligned}
$$

Here, the last inequality follows by using Theorem 2.3. This concludes the proof.

The following theorem gives an error estimate for the pressure.

Theorem 2.5. It holds

$$
\begin{aligned}
\left\|p-p_{h}\right\|_{M} \leq & C\left(\left\|p-R_{h} p\right\|_{M}+\left\|\mathbf{u}-P_{h} \mathbf{u}\right\|_{h}+\left\|\boldsymbol{\phi}-\bar{P}_{h} \boldsymbol{\phi}\right\|_{h}\right. \\
& \left.+\left\|E \boldsymbol{\phi}-\Pi_{h}(E \boldsymbol{\phi})\right\|_{Q}+\left\|\mathbf{y}-\Pi_{h} \mathbf{y}\right\|_{Q}\right) .
\end{aligned}
$$

Proof. The error term $\left\|p-p_{h}\right\|_{M}$ can be bounded by the sum of $\left\|p-R_{h} p\right\|_{M}$ and $\left\|R_{h} p-p_{h}\right\|_{M}$ with $R_{h}$ projection defined in (2.7a) $-(2.7 \mathrm{~b})$. Here it is sufficient to estimate $\left\|R_{h} p-p_{h}\right\|_{M}$ to get (2.24). From the inf-sup condition we have

$$
\bar{C}\left\|R_{h} p-p_{h}\right\|_{M} \leq \sup _{\mathbf{z}_{h} \in X_{h}} \frac{b_{h}\left(\mathbf{z}_{h}, R_{h} p-p_{h}\right)}{\left\|\mathbf{z}_{h}\right\|_{h}} .
$$

Use of (2.12) and Assumption II imply

$$
\begin{aligned}
\bar{C}\left\|R_{h} p-p_{h}\right\|_{M} & \leq \sup _{\mathbf{z}_{h} \in X_{h}} \frac{a_{h}\left(\mathbf{u}_{h}-P_{h} \mathbf{u}, \mathbf{z}_{h}\right)+\left\langle\mathbf{y}-\mathbf{y}_{h}, E_{h} \mathbf{z}_{h}\right\rangle_{Q}}{\left\|\mathbf{z}_{h}\right\|_{h}}, \\
& \leq C\left(\left\|\mathbf{u}_{h}-P_{h} \mathbf{u}\right\|_{h}+\left\|\mathbf{y}-\mathbf{y}_{h}\right\|_{Q}\right) .
\end{aligned}
$$

The split of term $\left\|\mathbf{u}_{h}-P_{h} \mathbf{u}\right\|_{h}$ as $\left\|\mathbf{u}_{h}-\mathbf{u}\right\|_{h}+\left\|\mathbf{u}-P_{h} \mathbf{u}\right\|_{h}$ and the use of estimates from (2.11) and (2.23) lead to (2.24). 
Similarly we can derive the following error estimates of adjoint pressure.

$$
\begin{aligned}
\left\|r-r_{h}\right\|_{M} \leq C\left(\left\|r-\bar{R}_{h} r\right\|_{M}+\left\|\mathbf{u}-P_{h} \mathbf{u}\right\|_{h}\right. & +\left\|\boldsymbol{\phi}-\bar{P}_{h} \boldsymbol{\phi}\right\|_{h} \\
& \left.+\left\|E \boldsymbol{\phi}-\Pi_{h}(E \boldsymbol{\phi})\right\|_{Q}+\left\|\mathbf{y}-\Pi_{h} \mathbf{y}\right\|_{Q}\right) .
\end{aligned}
$$

2.2. A posteriori Error Analysis. This subsection is devoted to a posteriori error analysis. Define reconstructions $R \mathbf{u} \in X, \bar{R} \phi \in X$ and $R_{0} p \in M, \bar{R}_{0} r \in M$ by

$$
\begin{aligned}
a(R \mathbf{u}, \mathbf{z})+b\left(\mathbf{z}, R_{0} p\right) & =\left\langle\mathbf{y}_{h}, E \mathbf{z}\right\rangle_{Q}+\langle\mathbf{f}, \mathbf{z}\rangle_{W} & & \text { for all } \mathbf{z} \in X, \\
b(R \mathbf{u}, w) & =0 & & \text { for all } w \in M, \\
a(\mathbf{z}, \bar{R} \phi)-b\left(\mathbf{z}, \bar{R}_{0} r\right) & =\left\langle\mathbf{u}_{h}-\mathbf{u}_{d}, \mathbf{z}\right\rangle_{W} & & \text { for all } \mathbf{z} \in X, \\
b(\bar{R} \phi, w) & =0 & & \text { for all } w \in M .
\end{aligned}
$$

The well-posedness of the above system (2.25a $-(2.25 \mathrm{~b})$ follows from the facts that the righthand side of (2.25a) is a bounded linear functional on $X$, the bilinear forms $a$ and $b$ are continuous, $a$ is $Z$-elliptic and $b$ is inf-sup stable, and hence the system (2.25a)-(2.25b) has a unique solution [19, pp. 81]. Similarly, the system (2.25c)-(2.25d $)$ is well-posed.

From the systems of equations (2.1) and (2.25), we have

$$
\begin{aligned}
a(\mathbf{u}-R \mathbf{u}, \mathbf{z})+b\left(\mathbf{z}, p-R_{0} p\right) & =\left\langle\mathbf{y}-\mathbf{y}_{h}, E \mathbf{z}\right\rangle_{Q} & & \text { for all } \mathbf{z} \in X, \\
b(\mathbf{u}-R \mathbf{u}, w) & =0 & & \text { for all } w \in M, \\
a(\mathbf{z}, \boldsymbol{\phi}-\bar{R} \boldsymbol{\phi})+b\left(\mathbf{z},-r+\bar{R}_{0} r\right) & =\left\langle\mathbf{u}-\mathbf{u}_{h}, \mathbf{z}\right\rangle_{W} & & \text { for all } \mathbf{z} \in X, \\
b(\boldsymbol{\phi}-\bar{R} \boldsymbol{\phi}, w) & =0 & & \text { for all } w \in M .
\end{aligned}
$$

Lemma 2.6. For all $\mathbf{x}_{h} \in Q_{a d}^{h}$, it holds

$\left\langle E_{h}(\bar{R} \boldsymbol{\phi}-\boldsymbol{\phi}), \mathbf{y}-\mathbf{y}_{h}\right\rangle_{Q} \geq-\left\langle E_{h}\left(\boldsymbol{\phi}_{h}-\bar{R} \boldsymbol{\phi}\right), \mathbf{y}-\mathbf{y}_{h}\right\rangle_{Q}+\left\langle E_{h} \boldsymbol{\phi}_{h}+\lambda \mathbf{y}_{h}, \mathbf{y}-\mathbf{x}_{h}\right\rangle_{Q}+\lambda\left\|\mathbf{y}-\mathbf{y}_{h}\right\|_{Q}^{2}$.

Proof. For any $\mathbf{x}_{h} \in Q_{a d}^{h}$, we have

$$
\left\langle E_{h} \boldsymbol{\phi}_{h}+\lambda \mathbf{y}_{h}, \mathbf{y}-\mathbf{y}_{h}\right\rangle_{Q}=\left\langle E_{h} \boldsymbol{\phi}_{h}+\lambda \mathbf{y}_{h}, \mathbf{y}-\mathbf{x}_{h}\right\rangle_{Q}+\left\langle E_{h} \boldsymbol{\phi}_{h}+\lambda \mathbf{y}_{h}, \mathbf{x}_{h}-\mathbf{y}_{h}\right\rangle_{Q}
$$

Since for all $\mathbf{x}_{h} \in Q_{a d}^{h}\left\langle E_{h} \phi_{h}+\lambda \mathbf{y}_{h}, \mathbf{x}_{h}-\mathbf{y}_{h}\right\rangle_{Q} \geq 0$, we have

$$
\left\langle E_{h} \phi_{h}+\lambda \mathbf{y}_{h}, \mathbf{y}-\mathbf{y}_{h}\right\rangle_{Q} \geq\left\langle E_{h} \phi_{h}+\lambda \mathbf{y}_{h}, \mathbf{y}-\mathbf{x}_{h}\right\rangle_{Q} .
$$

Substituting $\mathbf{x}_{h}=\mathbf{y}_{h} \in Q_{a d}^{h}\left(\subseteq Q_{a d}\right)$ in (2.1e) and $E_{h} \boldsymbol{\phi}=E \boldsymbol{\phi}$ for $\boldsymbol{\phi} \in X$, we get

$$
-\left\langle E_{h} \boldsymbol{\phi}+\lambda \mathbf{y}, \mathbf{y}-\mathbf{y}_{h}\right\rangle_{Q} \geq 0 \text {. }
$$

Adding (2.28) and (2.29) yields

$$
\left\langle E_{h}\left(\phi_{h}-\boldsymbol{\phi}\right)+\lambda\left(\mathbf{y}_{h}-\mathbf{y}\right), \mathbf{y}-\mathbf{y}_{h}\right\rangle_{Q} \geq\left\langle E_{h} \phi_{h}+\lambda \mathbf{y}_{h}, \mathbf{y}-\mathbf{x}_{h}\right\rangle_{Q} .
$$

Consider the left-hand side of (2.27): we have

$$
\left\langle E_{h}(\bar{R} \boldsymbol{\phi}-\boldsymbol{\phi}), \mathbf{y}-\mathbf{y}_{h}\right\rangle_{Q} \geq-\left\langle E_{h}\left(\boldsymbol{\phi}_{h}-\bar{R} \boldsymbol{\phi}\right), \mathbf{y}-\mathbf{y}_{h}\right\rangle_{Q}+\left\langle E_{h}\left(\boldsymbol{\phi}_{h}-\boldsymbol{\phi}\right), \mathbf{y}-\mathbf{y}_{h}\right\rangle_{Q} \text {. }
$$


A use of (2.30) in (2.31) concludes the proof.

Theorem 2.7. It holds

$$
\left\|\mathbf{y}-\mathbf{y}_{h}\right\|_{Q}+\|\mathbf{u}-R \mathbf{u}\|_{W} \leq C\left(\left\|E_{h} \phi_{h}-\Pi_{h}\left(E_{h} \phi_{h}\right)\right\|_{Q}+\left\|\phi_{h}-\bar{R} \phi\right\|_{h}+\left\|R \mathbf{u}-\mathbf{u}_{h}\right\|_{W}\right) .
$$

Proof. With the substitutions $\mathbf{z}=\boldsymbol{\phi}-\bar{R} \boldsymbol{\phi}$ in (2.26a) and $\mathbf{z}=\mathbf{u}-R \mathbf{u}$ in (2.26c), we have

$$
\begin{aligned}
a(\mathbf{u}-R \mathbf{u}, \boldsymbol{\phi}-\bar{R} \boldsymbol{\phi})+b\left(\boldsymbol{\phi}-\bar{R} \boldsymbol{\phi}, p-R_{0} p\right) & =\left\langle\mathbf{y}-\mathbf{y}_{h}, E(\boldsymbol{\phi}-\bar{R} \boldsymbol{\phi})\right\rangle_{Q}, \\
a(\mathbf{u}-R \mathbf{u}, \boldsymbol{\phi}-\bar{R} \boldsymbol{\phi})+b\left(\mathbf{u}-R \mathbf{u},-r+\bar{R}_{0} r\right) & =\left\langle\mathbf{u}-\mathbf{u}_{h}, \mathbf{u}-R \mathbf{u}\right\rangle_{W} .
\end{aligned}
$$

Since $b\left(\phi-\bar{R} \phi, p-R_{0} p\right)=0$ and $b\left(\mathbf{u}-R \mathbf{u},-r+\bar{R}_{0} r\right)=0$ from (2.26d) and (2.26b), the subtraction of (2.34) from (2.33) yields

$$
\left\langle\mathbf{y}-\mathbf{y}_{h}, E(\boldsymbol{\phi}-\bar{R} \boldsymbol{\phi})\right\rangle_{Q}-\left\langle\mathbf{u}-\mathbf{u}_{h}, \mathbf{u}-R \mathbf{u}\right\rangle_{W}=0
$$

and

$$
\left\langle\mathbf{y}-\mathbf{y}_{h}, E(\bar{R} \boldsymbol{\phi}-\boldsymbol{\phi})\right\rangle_{Q}+\|\mathbf{u}-R \mathbf{u}\|_{W}^{2}=-\left\langle R \mathbf{u}-\mathbf{u}_{h}, \mathbf{u}-R \mathbf{u}\right\rangle_{W} .
$$

From Lemma 2.6 and (2.35), we have

$\lambda\left\|\mathbf{y}-\mathbf{y}_{h}\right\|_{Q}^{2}+\|\mathbf{u}-R \mathbf{u}\|_{W}^{2} \leq-\left\langle E_{h} \phi_{h}+\lambda \mathbf{y}_{h}, \mathbf{y}-\mathbf{x}_{h}\right\rangle_{Q}+\left\langle E_{h}(\bar{R} \boldsymbol{\phi}-\boldsymbol{\phi}), \mathbf{y}-\mathbf{y}_{h}\right\rangle_{Q}$

$$
+\left\langle E_{h}\left(\phi_{h}-\bar{R} \phi\right), \mathbf{y}-\mathbf{y}_{h}\right\rangle_{Q}-\left\langle\mathbf{y}-\mathbf{y}_{h}, E(\bar{R} \boldsymbol{\phi}-\boldsymbol{\phi})\right\rangle_{Q}-\left\langle R \mathbf{u}-\mathbf{u}_{h}, \mathbf{u}-R \mathbf{u}\right\rangle_{W}
$$

Taking the first term on the right-hand side of (2.36) with $\mathbf{x}_{h}=\Pi_{h} \mathbf{y} \in Q_{a d}^{h}$ and using Assumption III show

$$
\begin{aligned}
\left\langle E_{h} \phi_{h}+\lambda \mathbf{y}_{h}, \mathbf{y}-\Pi_{h} \mathbf{y}\right\rangle_{Q} & =\left\langle E_{h} \phi_{h}, \mathbf{y}-\Pi_{h} \mathbf{y}\right\rangle_{Q},=\left\langle E_{h} \phi_{h}-\Pi_{h}\left(E_{h} \phi_{h}\right), \mathbf{y}-\Pi_{h} \mathbf{y}\right\rangle_{Q} \\
& =\left\langle E_{h} \phi_{h}-\Pi_{h}\left(E_{h} \phi_{h}\right), \mathbf{y}-\mathbf{y}_{h}\right\rangle_{Q} .
\end{aligned}
$$

The substitution of (2.37) in (2.36), a use of Assumption II and the Young inequality result in

$$
\begin{aligned}
\lambda\left\|\mathbf{y}-\mathbf{y}_{h}\right\|_{Q}^{2}+\|\mathbf{u}-R \mathbf{u}\|_{W}^{2} \leq & \left\langle E_{h} \boldsymbol{\phi}_{h}-\Pi_{h}\left(E_{h} \boldsymbol{\phi}_{h}\right), \mathbf{y}-\mathbf{y}_{h}\right\rangle_{Q}+\left\langle E_{h}\left(\boldsymbol{\phi}_{h}-\bar{R} \boldsymbol{\phi}\right), \mathbf{y}-\mathbf{y}_{h}\right\rangle_{Q} \\
& -\left\langle R \mathbf{u}-\mathbf{u}_{h}, \mathbf{u}-R \mathbf{u}\right\rangle_{W}, \\
\leq & \frac{1}{2 \lambda}\left\|E_{h} \boldsymbol{\phi}_{h}-\Pi_{h}\left(E_{h} \boldsymbol{\phi}_{h}\right)\right\|_{Q}^{2}+\frac{\lambda}{4}\left\|\mathbf{y}-\mathbf{y}_{h}\right\|_{Q}^{2}+\frac{C}{2 \lambda}\left\|\phi_{h}-\bar{R} \boldsymbol{\phi}\right\|_{h}^{2}, \\
& +\frac{\lambda}{4}\left\|\mathbf{y}-\mathbf{y}_{h}\right\|_{Q}^{2}+\frac{1}{2}\left\|R \mathbf{u}-\mathbf{u}_{h}\right\|_{W}^{2}+\frac{1}{2}\|\mathbf{u}-R \mathbf{u}\|_{W}^{2} .
\end{aligned}
$$

Rearrangement of the terms in (2.38) leads to the estimates (2.32).

Theorem 2.8. It holds

$$
\left\|\mathbf{u}-\mathbf{u}_{h}\right\|_{h} \leq C\left(\left\|R \mathbf{u}-\mathbf{u}_{h}\right\|_{h}+\left\|E_{h} \phi_{h}-\Pi_{h}\left(E_{h} \phi_{h}\right)\right\|_{Q}+\left\|\phi_{h}-\bar{R} \phi\right\|_{h}\right),
$$

and

$$
\left\|\phi-\phi_{h}\right\|_{h} \leq C\left(\left\|\phi_{h}-\bar{R} \phi\right\|_{h}+\left\|R \mathbf{u}-\mathbf{u}_{h}\right\|_{h}+\left\|E_{h} \phi_{h}-\Pi_{h}\left(E_{h} \phi_{h}\right)\right\|_{Q}\right)
$$


Proof. To prove (2.39), it is sufficient to estimate $\|\mathbf{u}-R \mathbf{u}\|_{h}$ as the error term $\left\|\mathbf{u}-\mathbf{u}_{h}\right\|_{h}$ can be bounded by the sum of $\|\mathbf{u}-R \mathbf{u}\|_{h}$ and $\left\|R \mathbf{u}-\mathbf{u}_{h}\right\|_{h}$. Putting $\mathbf{z}=\mathbf{u}-R \mathbf{u}$ in (2.26a), gives

$$
a(\mathbf{u}-R \mathbf{u}, \mathbf{u}-R \mathbf{u})=\left\langle\mathbf{y}-\mathbf{y}_{h}, E(\mathbf{u}-R \mathbf{u})\right\rangle_{Q} .
$$

The Z-ellipticity of $a(\cdot, \cdot)$ and Assumption II imply

$$
\|\mathbf{u}-R \mathbf{u}\|_{X} \leq C\left\|\mathbf{y}-\mathbf{y}_{h}\right\|_{Q} .
$$

Since $\mathbf{u}-R \mathbf{u} \in X$ and $\|\mathbf{u}-R \mathbf{u}\|_{X}=\|\mathbf{u}-R \mathbf{u}\|_{h}$, we have $\|\mathbf{u}-R \mathbf{u}\|_{h} \leq C\left\|\mathbf{y}-\mathbf{y}_{h}\right\|_{Q}$. Hence

$$
\left\|\mathbf{u}-\mathbf{u}_{h}\right\|_{h} \leq C\left\|\mathbf{y}-\mathbf{y}_{h}\right\|_{Q}+\left\|R \mathbf{u}-\mathbf{u}_{h}\right\|_{h} .
$$

The estimates for $\left\|\mathbf{y}-\mathbf{y}_{h}\right\|_{Q}$ from Theorem 2.7 and Assumption I lead to

$$
\left\|\mathbf{u}-\mathbf{u}_{h}\right\|_{h} \leq C\left(\left\|R \mathbf{u}-\mathbf{u}_{h}\right\|_{h}+\left\|E_{h} \phi_{h}-\Pi_{h}\left(E_{h} \phi_{h}\right)\right\|_{Q}+\left\|\phi_{h}-\bar{R} \boldsymbol{\phi}\right\|_{h}\right) .
$$

Now

$$
\left\|\phi-\phi_{h}\right\|_{h} \leq\|\phi-\bar{R} \phi\|_{h}+\left\|\bar{R} \phi-\phi_{h}\right\|_{h} .
$$

Putting $\mathbf{z}=\phi-\bar{R} \phi$ in (2.26c) and using assumption (2.5), we conclude that

$$
\|\phi-\bar{R} \phi\|_{h} \leq C\left\|\mathbf{u}-\mathbf{u}_{h}\right\|_{h},
$$

which implies that

$$
\left\|\phi-\phi_{h}\right\|_{h} \leq C\left\|\mathbf{u}-\mathbf{u}_{h}\right\|_{h}+\left\|\bar{R} \phi-\phi_{h}\right\|_{h} .
$$

Finally, substitution of the bounds of $\left\|\mathbf{u}-\mathbf{u}_{h}\right\|_{h}$ from (2.42) in (2.43) leads to (2.40).

Theorem 2.9. It holds,

$$
\left\|p-p_{h}\right\|_{M} \leq C\left(\left\|R_{0} p-p_{h}\right\|_{M}+\left\|E_{h} \boldsymbol{\phi}_{h}-\Pi_{h}\left(E_{h} \boldsymbol{\phi}_{h}\right)\right\|_{Q}+\left\|R \mathbf{u}-\mathbf{u}_{h}\right\|_{W}+\left\|\boldsymbol{\phi}_{h}-\bar{R} \boldsymbol{\phi}\right\|_{h}\right) .
$$

Proof. From the inf-sup condition, we have

$$
\beta\left\|p-R_{0} p\right\|_{M} \leq \sup _{\mathbf{z} \in X} \frac{b\left(\mathbf{z}, p-R_{0} p\right)}{\|\mathbf{z}\|_{X}} .
$$

Use of (2.26a) on the right-hand side of (2.44) shows

$$
\begin{aligned}
\beta\left\|p-R_{0} p\right\|_{M} & \leq \sup _{\mathbf{z} \in X} \frac{b\left(\mathbf{z}, p-R_{0} p\right)}{\|\mathbf{z}\|_{X}} \leq \sup _{\mathbf{z} \in X} \frac{\left\langle\mathbf{y}-\mathbf{y}_{h}, E \mathbf{z}\right\rangle_{Q}+a(R \mathbf{u}-\mathbf{u}, \mathbf{z})}{\|\mathbf{z}\|_{X}} \\
& \leq C\left(\left\|\mathbf{y}-\mathbf{y}_{h}\right\|_{Q}+\|R \mathbf{u}-\mathbf{u}\|_{X}\right) .
\end{aligned}
$$

Since from (2.41) we have $\|R \mathbf{u}-\mathbf{u}\|_{X}=\|R \mathbf{u}-\mathbf{u}\|_{h} \leq C\left\|\mathbf{y}-\mathbf{y}_{h}\right\|_{Q}$. The triangle inequality and (2.45) show

$$
\left\|p-p_{h}\right\|_{M} \leq\left\|p-R_{0} p\right\|_{M}+\left\|R_{0} p-p_{h}\right\|_{M} \leq C\left(\left\|\mathbf{y}-\mathbf{y}_{h}\right\|_{Q}+\left\|R_{0} p-p_{h}\right\|_{M}\right) .
$$

Substitution of the expression $\left\|\mathbf{y}-\mathbf{y}_{h}\right\|_{Q}$ from (2.32) in the above equation results in

$$
\left\|p-p_{h}\right\|_{M} \leq C\left(\left\|R_{0} p-p_{h}\right\|_{M}+\left\|E_{h} \phi_{h}-\Pi_{h}\left(E_{h} \phi_{h}\right)\right\|_{Q}+\left\|R \mathbf{u}-\mathbf{u}_{h}\right\|_{h}+\left\|\phi_{h}-\bar{R} \phi\right\|_{h}\right) .
$$

This concludes the proof. 
Theorem 2.10. There holds,

$$
\left\|r-r_{h}\right\|_{M} \leq C\left(\left\|\bar{R}_{0} r-r_{h}\right\|_{M}+\left\|E_{h} \phi_{h}-\Pi_{h}\left(E_{h} \phi_{h}\right)\right\|_{Q}+\left\|R \mathbf{u}-\mathbf{u}_{h}\right\|_{h}+\left\|\phi_{h}-\bar{R} \phi\right\|_{h}\right) .
$$

Proof. The proof follows by the similar steps as in Theorem 2.9.

Remark 2.11. Note that the upper bounds of the error terms in Theorem 2.8, 2.9 and 2.10 contain some non-computable terms, for example, $\left\|R \mathbf{u}-\mathbf{u}_{h}\right\|_{h},\left\|\phi_{h}-\bar{R} \phi\right\|_{h},\left\|R_{0} p-p_{h}\right\|_{M}$ and $\left\|\bar{R}_{0} r-r_{h}\right\|_{M}$. These terms can be estimated in terms of the computable quantities so-called estimator, as it has been shown in Section 4 .

\section{Model PROBlems}

This section deals with two model problems: A distributed control problem and a Neumann boundary control problem. We will see the application of the abstract framework from Section 2 to these model problems. We start with some notation used throughout this article. Let $\Omega \subset \mathbb{R}^{2}$ be a bounded polyhedral domain with boundary $\Gamma$. The spaces $L^{2}(\Omega)$ and $H^{1}(\Omega)$ are standard Sobolev spaces. The vector valued version of $L^{2}(\Omega)$ and $H^{1}(\Omega)$ are denoted by $\left[L^{2}(\Omega)\right]^{2}$ and $\left[H^{1}(\Omega)\right]^{2}$, respectively, and the $L^{2}$-norm on $\Omega$ is denoted by $\|\cdot\|_{0, \Omega}$. The subspace of $L^{2}(\Omega)$ with zero mean functions is defined by $L_{0}^{2}(\Omega):=\left\{q \in L^{2}(\Omega): \int_{\Omega} q d x=0\right\}$ and $H_{0}^{1}(\Omega)$ is the subspace of $H^{1}(\Omega)$ with zero trace functions.

\subsection{Distributed control problem. Set}

$$
X=\left[H_{0}^{1}(\Omega)\right]^{2}, W=\left[L^{2}(\Omega)\right]^{2}, M=L_{0}^{2}(\Omega) \text { and } Q=\left[L^{2}(\Omega)\right]^{2} .
$$

The map $E: X \rightarrow Q$ is the inclusion map. Given $\mathbf{u}_{d}$ in $\left[L^{2}(\Omega)\right]^{2}$, define the quadratic functional $J:\left[H_{0}^{1}(\Omega)\right]^{2} \times\left[L^{2}(\Omega)\right]^{2} \rightarrow \mathbb{R}$ by

$$
J(\mathbf{v}, \mathbf{x})=\frac{1}{2}\left\|\mathbf{v}-\mathbf{u}_{d}\right\|_{0, \Omega}^{2}+\frac{\lambda}{2}\|\mathbf{x}\|_{0, \Omega}^{2} \mathbf{v} \in\left[H_{0}^{1}(\Omega)\right]^{2}, \mathbf{x} \in\left[L^{2}(\Omega)\right]^{2} .
$$

For given $\mathbf{y}_{a}, \mathbf{y}_{b} \in[\mathbb{R} \cup\{ \pm \infty\}]^{2}$ with $\mathbf{y}_{a}<\mathbf{y}_{b}$, define the admissible set of controls by

$$
Q_{d}=\left\{\mathbf{x} \in\left[L^{2}(\Omega)\right]^{2} \mid \mathbf{y}_{a} \leq \mathbf{x} \leq \mathbf{y}_{b}\right\}, \quad Q_{a d}:=Q_{d}
$$

Consider the optimal control problem of finding $(\mathbf{u}, \mathbf{y}) \in X \times Q_{d}$ such that,

$$
J(\mathbf{u}, \mathbf{y})=\inf _{\mathbf{v} \in\left[H_{0}^{1}(\Omega)\right]^{2}, \mathbf{x} \in Q_{d}} J(\mathbf{v}, \mathbf{x}),
$$

subject to the condition that $\mathbf{v}$ and $\mathbf{x}$ are such that $(\mathbf{v}, p, \mathbf{x})$ satisfies: for all $(\mathbf{z}, w) \in X \times M$

$$
a(\mathbf{v}, \mathbf{z})+b(\mathbf{z}, p)=\langle\mathbf{x}, \mathbf{z}\rangle_{\left[L^{2}(\Omega)\right]^{2}}+\langle\mathbf{f}, \mathbf{z}\rangle_{\left[L^{2}(\Omega)\right]^{2}} \quad \text { and } \quad b(\mathbf{v}, w)=0
$$

where $a(\mathbf{v}, \mathbf{z})=\int_{\Omega} \nabla \mathbf{v}: \nabla \mathbf{z} \mathrm{dx}, b(\mathbf{z}, p)=-\int_{\Omega} p \nabla \cdot \mathbf{z} \mathrm{dx}$, and the matrix product $A: B:=$ $\sum_{i, j=1}^{n} a_{i j} b_{i j}$ when $A=\left(a_{i j}\right)_{1 \leq i, j \leq n}$ and $B=\left(b_{i j}\right)_{1 \leq i, j \leq n}$.

The optimal solution $(\mathbf{u}, p, \mathbf{y}) \in X \times M \times Q_{d}$, satisfy the following: for all $(\mathbf{z}, w) \in X \times M$

$$
a(\mathbf{u}, \mathbf{z})+b(\mathbf{z}, p)=\langle\mathbf{y}, \mathbf{z}\rangle_{\left[L^{2}(\Omega)\right]^{2}}+\langle\mathbf{f}, \mathbf{z}\rangle_{\left[L^{2}(\Omega)\right]^{2}} \text { and } b(\mathbf{u}, w)=0 .
$$


Note that the model problem (3.4) has a unique solution $(\mathbf{v}, p) \in\left[H_{0}^{1}(\Omega)\right]^{2} \times L_{0}^{2}(\Omega)$ for given $\mathbf{x} \in\left[L^{2}(\Omega)\right]^{2}\left[19\right.$, pp. 81]. We can set this correspondence as $S^{\mathbf{v}} \mathbf{x}=\mathbf{v}$ and $S^{p} \mathbf{x}=p$ and using the stability estimates of $\mathbf{v}$ and $p$ one can show that $S^{\mathbf{v}}:\left[L^{2}(\Omega)\right]^{2} \rightarrow\left[L^{2}(\Omega)\right]^{2}$ and $S^{p}:\left[L^{2}(\Omega)\right]^{2} \rightarrow L_{0}^{2}(\Omega)$ are continuous affine operators. Then the minimization problem (3.3) becomes

$$
j(\mathbf{y})=\inf _{\mathbf{x} \in Q_{d}} j(\mathbf{x}),
$$

where

$$
j(\mathbf{x})=\frac{1}{2}\left\|S^{\mathbf{v}} \mathbf{x}-\mathbf{u}_{d}\right\|_{0, \Omega}^{2}+\frac{\lambda}{2}\|\mathbf{x}\|_{0, \Omega}^{2} .
$$

Using the theory of elliptic optimal control problems, the following proposition on the existence and uniqueness of the solution can be proved and the optimality condition can be derived [34, pp. 50].

Proposition 3.1. The control problem (3.6) has a unique solution $\mathbf{y}$ and correspondingly there exist a unique state $(\mathbf{u}, p)=\left(S^{\mathbf{u}} \mathbf{y}, S^{p} \mathbf{y}\right)$ of (3.5). Furthermore, by introducing the adjoint state $(\phi, r) \in X \times M$ such that, for all $(\mathbf{z}, w) \in X \times M$

$$
a(\mathbf{z}, \boldsymbol{\phi})-b(\mathbf{z}, r)=\left\langle\mathbf{u}-\mathbf{u}_{d}, \mathbf{z}\right\rangle_{\left[L^{2}(\Omega)\right]^{2}}, \quad b(\boldsymbol{\phi}, w)=0,
$$

the optimality condition that $j^{\prime}(\mathbf{y})(\mathbf{x}-\mathbf{y}) \geq 0, \forall \mathbf{x} \in Q_{d}$, can be expressed as

$$
\langle\phi+\lambda \mathbf{y}, \mathbf{x}-\mathbf{y}\rangle_{\left[L^{2}(\Omega)\right]^{2}} \geq 0 \text { for all } \mathbf{x} \in Q_{d} .
$$

The strong formulation of the optimality conditions satisfied by $((\mathbf{u}, p),(\boldsymbol{\phi}, r), \mathbf{y})$ is given by the following system of equations:

$$
\begin{aligned}
-\Delta \mathbf{u}+\nabla p & =\mathbf{y}+\mathbf{f}, & \nabla \cdot \mathbf{u}=0 \text { in } \Omega, & \mathbf{u}=\mathbf{0} \text { on } \partial \Omega, \\
-\Delta \phi-\nabla r & =\mathbf{u}-\mathbf{u}_{d}, & \nabla \cdot \boldsymbol{\phi}=0 \text { in } \Omega, & \boldsymbol{\phi}=\mathbf{0} \text { on } \partial \Omega, \\
\mathbf{y} & =\Pi_{\left[\mathbf{y}_{a}, \mathbf{y}_{b}\right]}\left(-\frac{\boldsymbol{\phi}}{\lambda}\right) \quad \text { on } \Omega, & &
\end{aligned}
$$

where $\Pi_{[a, b]} g(x)=\min \{b, \max \{a, g(x)\}\}$.

\subsection{Neumann boundary control problem. Set}

$$
X=\left[H^{1}(\Omega) \cap L_{0}^{2}(\Omega)\right]^{2}, W=\left[L^{2}(\Omega)\right]^{2}, M=L^{2}(\Omega) \text { and } Q=\left[L^{2}(\Gamma)\right]^{2} .
$$

The map $E: X \rightarrow Q$ is the trace map. Define the quadratic functional $J: X \times\left[L_{2}(\Gamma)\right]^{2} \rightarrow \mathbb{R}$ by

$$
J(\mathbf{v}, \mathbf{x})=\frac{1}{2}\left\|\mathbf{v}-\mathbf{u}_{d}\right\|_{0, \Omega}^{2}+\frac{\lambda}{2}\|\mathbf{x}\|_{0, \Gamma}^{2} \quad \mathbf{v} \in X, \mathbf{x} \in\left[L^{2}(\Gamma)\right]^{2} .
$$


For given $\mathbf{y}_{a}, \mathbf{y}_{b} \in[\mathbb{R} \cup\{ \pm \infty\}]^{2}$ with $\mathbf{y}_{a}<\mathbf{y}_{b}$, and $\mathbf{y}_{a} \leq-\frac{1}{|\Gamma|} \int_{\Omega} \mathbf{f} \mathrm{dx} \leq \mathbf{y}_{b}$ define the admissible set of controls by

$$
Q_{b}=\left\{\mathbf{x} \in\left[L_{2}(\Gamma)\right]^{2} \mid \int_{\Gamma} \mathbf{x} \mathrm{ds}+\int_{\Omega} \mathbf{f} \mathrm{dx}=\mathbf{0}, \quad \mathbf{y}_{a} \leq \mathbf{x} \leq \mathbf{y}_{b}\right\}, \quad Q_{a d}:=Q_{d} .
$$

Consider the optimal control problem of finding $(\mathbf{u}, \mathbf{y}) \in X \times Q_{b}$ such that,

$$
J(\mathbf{u}, \mathbf{y})=\inf _{\mathbf{v} \in X, \mathbf{x} \in Q_{b}} J(\mathbf{v}, \mathbf{x})
$$

subject to the condition that $\mathbf{v}$ and $\mathbf{x}$ are such that $(\mathbf{v}, p, \mathbf{x})$ satisfies: for all $(\mathbf{z}, w) \in X \times M$

$$
a(\mathbf{v}, \mathbf{z})+b(\mathbf{z}, p)=\langle\mathbf{x}, \mathbf{z}\rangle_{\left[L^{2}(\Gamma)\right]^{2}}+\langle\mathbf{f}, \mathbf{z}\rangle_{\left[L^{2}(\Omega)\right]^{2}}, b(\mathbf{v}, w)=0 .
$$

where both the bilinear forms $a(\mathbf{v}, \mathbf{z})$ and $b(\mathbf{z}, p)$ are same as that of the distributed case.

The optimal solution $(\mathbf{u}, p, \mathbf{y}) \in X \times M \times Q_{b}$ satisfies

$$
\begin{aligned}
a(\mathbf{u}, \mathbf{z})+b(\mathbf{z}, p) & =\langle\mathbf{y}, \mathbf{z}\rangle_{\left[L_{2}(\Gamma)\right]^{2}}+\langle\mathbf{f}, \mathbf{z}\rangle_{\left[L^{2}(\Omega)\right]^{2}} \text { for all } \mathbf{z} \in X, \\
b(\mathbf{u}, w) & =0 \text { for all } w \in M .
\end{aligned}
$$

Note that the model problem (3.14) has a unique solution $(\mathbf{v}, p) \in X \times M$ for given $\mathbf{x} \in$ $\left[L^{2}(\Gamma)\right]^{2}\left[19\right.$. We can set this correspondence as $S^{\mathbf{v}} \mathbf{x}=\mathbf{v}$ and $S^{p} \mathbf{X}=p$ and using the stability estimates of $\mathbf{v}$ and $p$, one can show that $S^{\mathbf{v}}:\left[L^{2}(\Gamma)\right]^{2} \rightarrow\left[L^{2}(\Omega)\right]^{2}$ and $S^{p}:\left[L^{2}(\Gamma)\right]^{2} \rightarrow L^{2}(\Omega)$ are continuous affine operators. Then the minimization problem (3.13) becomes

$$
j(\mathbf{y})=\inf _{\mathbf{x} \in Q_{b}} j(\mathbf{x}),
$$

where

$$
j(\mathbf{x})=\frac{1}{2}\left\|S^{\mathbf{v}} \mathbf{x}-\mathbf{u}_{d}\right\|_{0, \Omega}^{2}+\frac{\lambda}{2}\|\mathbf{x}\|_{0, \Gamma}^{2} .
$$

The following proposition on the existence and uniqueness of the solution and the optimality condition can be derived using the theory of elliptic optimal control problems [34, pp. 50].

Proposition 3.2. The control problem (3.16) has a unique solution $\mathbf{y}$ and correspondingly there exist a unique state $(\mathbf{u}, p)=\left(S^{\mathbf{v}} \mathbf{y}, S^{p} \mathbf{y}\right)$ of (3.15). Furthermore, by introducing the adjoint state $(\phi, r) \in X \times M$ such that, for all $(\mathbf{z}, w) \in X \times M$

$$
a(\mathbf{z}, \mathbf{u})-b(\mathbf{z}, r)=\left\langle\mathbf{u}-\mathbf{u}_{d}, \mathbf{z}\right\rangle_{\left[L^{2}(\Omega)\right]^{2}}, \quad b(\boldsymbol{\phi}, w)=0,
$$

the optimality condition that $j^{\prime}(\mathbf{y})(\mathbf{x}-\mathbf{y}) \geq 0$, for all $\mathbf{x} \in Q_{b}$, can be expressed as

$$
\langle\boldsymbol{\phi}+\lambda \mathbf{y}, \mathbf{x}-\mathbf{y}\rangle_{\left[L_{2}(\Gamma)\right]^{2}} \geq 0 \text { for all } \mathbf{x} \in Q_{b}
$$

The strong formulation of the optimality conditions satisfied by $((\mathbf{u}, p),(\phi, r)$, $\mathbf{y})$ is given by the following system of equations:

$$
-\Delta \mathbf{u}+\nabla p=\mathbf{f}, \quad \nabla \cdot \mathbf{u}=0 \text { in } \Omega, \quad \frac{\partial \mathbf{u}}{\partial \mathbf{n}}-p \mathbf{n}=\mathbf{y} \text { on } \partial \Omega,
$$




$$
\begin{aligned}
-\Delta \phi-\nabla r & =\mathbf{u}-\mathbf{u}_{d}, \quad \nabla \cdot \boldsymbol{\phi}=0 \text { in } \Omega, \quad \frac{\partial \boldsymbol{\phi}}{\partial \mathbf{n}}+r \mathbf{n}=0 \text { on } \partial \Omega, \\
\mathbf{y} & =\prod_{\left[\mathbf{y}_{a}, \mathbf{y}_{b}\right]}\left(-\frac{\boldsymbol{\phi}}{\lambda}\right) \text { on } \partial \Omega,
\end{aligned}
$$

where $\Pi_{[a, b]} g(x)=\min \{b, \max \{a, g(x)\}\}$.

\section{Discrete Problems}

In this section, we will discuss finite element formulations for the model problems studied in the last section. First, we will consider Crouzeix-Raviart finite element space for velocity and piecewise constant polynomial space for pressure. Secondly, we will discuss the discontinuous Galerkin (DG) method with piecewise linear space and piecewise constant space for velocity and pressure approximation, respectively. We start this section with the notation which has used throughout the article.

4.1. Notation. Let $\mathcal{T}_{h}$ be a regular triangulation of $\Omega$ into triangles such that $\cup_{T \in \mathcal{T}_{h}} T=\bar{\Omega}$. Denote the set of all interior edges of $\mathcal{T}_{h}$ by $\mathcal{E}_{h}^{i}$, the set of boundary edges by $\mathcal{E}_{h}^{b}$, and define $\mathcal{E}_{h}=\mathcal{E}_{h}^{i} \cup \mathcal{E}_{h}^{b}$. Let $h_{T}:=\operatorname{diam}(T)$ and $h=\max \left\{h_{T}: T \in \mathcal{T}_{h}\right\}$. The length of any edge $e \in \mathcal{E}_{h}$ will be denoted by $h_{e}$. Let us define a broken Sobolev space

$$
H^{1}\left(\Omega, \mathcal{T}_{h}\right)=\left\{v \in L^{2}(\Omega):\left.v\right|_{T} \in H^{1}(T) \text { for all } T \in \mathcal{T}_{h}\right\} .
$$

Denote the norm and the semi-norm on $H^{k}(D)(k \geq 0)$ for any domain $D \subseteq \mathbb{R}^{2}$ by $\|v\|_{k, D}$ and $|v|_{k, D}$. In the problem setting, we require jump and mean definitions of discontinuous functions, vector functions and tensors. For any $e \in \mathcal{E}_{h}^{i}$, there are two triangles $T_{+}$and $T_{-}$ such that $e=\partial T_{+} \cap \partial T_{-}$. Let $\mathbf{n}_{+}$be the unit normal of $e$ pointing from $T_{+}$to $T_{-}$and let $\mathbf{n}_{-}=-\mathbf{n}_{+}$(cf. Fig 4.1). For any $v \in H^{1}\left(\Omega, \mathcal{T}_{h}\right)$, we define the jump and mean of $v$ on an edge $e$ by

$$
\llbracket v \rrbracket=v_{+} \mathbf{n}_{+}+v_{-} \mathbf{n}_{-} \text {and }\{\{v\}\}=\frac{1}{2}\left(v_{+}+v_{-}\right) \text {respectively, }
$$

where $v_{ \pm}=\left.v\right|_{T_{ \pm}}$.

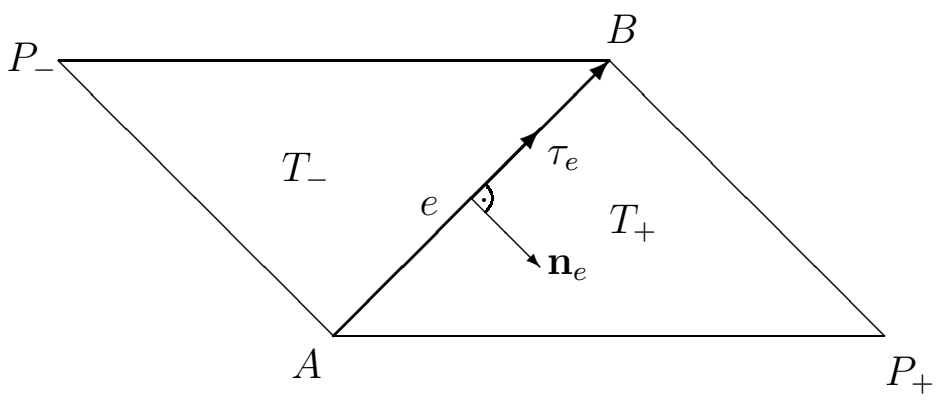

FiguRE 4.1. Here $T_{-}$and $T_{+}$are the two neighboring triangles that share the edge $e=\partial T_{-} \cap \partial T_{+}$with initial node $A$ and end node $B$ and unit normal $\mathbf{n}_{e}$. The orientation of $\mathbf{n}_{e}=\mathbf{n}_{-}=-\mathbf{n}_{+}$equals the outer normal of $T_{-}$, and hence, points into $T_{+}$. 
For $\mathbf{v} \in\left[H^{1}\left(\Omega, \mathcal{T}_{h}\right)\right]^{2}$ we define the jump and mean of $\mathbf{v}$ on $e \in \mathcal{E}_{h}^{i}$. by

$$
\llbracket \mathbf{v} \rrbracket=\mathbf{v}_{+} \cdot \mathbf{n}_{+}+\mathbf{v}_{-} \cdot \mathbf{n}_{-} \text {and }\{\{\mathbf{v}\}\}=\frac{1}{2}\left(\mathbf{v}_{+}+\mathbf{v}_{-}\right) \text {respectively. }
$$

We also require the full jump of vector valued functions. For $\mathbf{v} \in\left[H^{1}\left(\Omega, \mathcal{T}_{h}\right)\right]^{2}$, we define the full jump by

$$
\underline{\llbracket \mathbf{v} \rrbracket}=\mathbf{v}_{+} \otimes \mathbf{n}_{+}+\mathbf{v}_{-} \otimes \mathbf{n}_{-},
$$

where for two vectors in Cartesian coordinates $\mathbf{a}=\left(a_{i}\right)$ and $\mathbf{b}=\left(b_{j}\right)$, we define the matrix $\mathbf{a} \otimes \mathbf{b}=\left[a_{i} b_{j}\right]_{1 \leq i, j \leq 2}$. Similarly, for tensors $\tau \in\left[H^{1}\left(\Omega, \mathcal{T}_{h}\right)\right]^{2 \times 2}$, the jump and mean on $e \in \mathcal{E}_{h}^{i}$ are defined by

$$
\llbracket \tau \rrbracket=\tau_{+} \mathbf{n}_{+}+\tau_{-} \mathbf{n}_{-} \text {and }\{\{\tau\}\}=\frac{1}{2}\left(\tau_{+}+\tau_{-}\right) .
$$

For notational convenience, we also define the jump and mean on the boundary faces $e \in \mathcal{E}_{h}^{b}$ by modifying them appropriately. We use the definition of jump by understanding that $v_{-}=0$ (similarly, $\mathbf{v}_{-}=0$ and $\tau_{-}=0$ ) and the definition of mean by understanding that $v_{-}=v_{+}$(similarly, $\mathbf{v}_{-}=\mathbf{v}_{+}$and $\left.\tau_{-}=\tau_{+}\right)$.

Define $\mathbb{P}_{m}(T)$ to be the space of polynomials of degree at most $m(\geq 0)$ defined on the triangle $T$. The discontinuous finite element space is

$$
\mathbb{P}_{m}\left(\mathcal{T}_{h}\right):=\left\{v_{h} \in L^{2}(\Omega)\left|\forall T \in \mathcal{T}_{h}, v_{h}\right|_{T} \in \mathbb{P}_{m}(T)\right\}
$$

The lowest-order Crouzeix-Raviart (CR) spaces are defined as

$$
\begin{aligned}
\mathrm{CR}^{1}\left(\mathcal{T}_{h}\right):=\left\{\mathbf{v}_{h} \in\left[L^{2}(\Omega)\right]^{2}:\right. & \forall T \in \mathcal{T}_{h},\left.\mathbf{v}_{h}\right|_{T} \in\left[\mathbb{P}_{1}(T)\right]^{2}, \\
& \left.v_{h} \text { continuous at midpoint of edge } e \text { for all } e \in \mathcal{E}_{h}^{i}\right\} .
\end{aligned}
$$

and $\mathrm{CR}_{0}^{1}\left(\mathcal{T}_{h}\right):=\left\{\mathbf{v}_{h} \in \mathrm{CR}^{1}\left(\mathcal{T}_{h}\right): \mathbf{v}_{h}(\operatorname{mid} e)=\mathbf{0}\right.$ for all $\left.e \in \mathcal{E}_{h}^{b}\right\}$

Define the oscillation of given functions $\mathbf{f}, \mathbf{u}_{d} \in\left[L^{2}(T)\right]^{2}$ by

$$
\operatorname{osc}(\mathbf{f}, T)=h_{T} \min _{\mathbf{f}_{h} \in\left[\mathbb{P}_{0}(T)\right]^{2}}\left\|\mathbf{f}-\mathbf{f}_{h}\right\|_{0, T} \text { and } \operatorname{osc}\left(\mathbf{u}_{d}, T\right)=h_{T} \min _{\mathbf{g}_{h} \in\left[\mathbb{P}_{0}(T)\right]^{2}}\left\|\mathbf{u}_{d}-\mathbf{g}_{h}\right\|_{0, T} .
$$

We will also need the following inverse estimates [7]:

Lemma 4.1. There exist a constant $C_{m}$ such that for all $v \in \mathbb{P}_{m}(T)$ one has

$$
\|\nabla v\|_{0, T} \leq C_{m} h_{T}^{-1}\|v\|_{0, T}
$$

and

$$
\|v\|_{0, \partial T} \leq C_{m} h_{T}^{-\frac{1}{2}}\|v\|_{0, T}
$$

4.2. Nonconforming FEM $\left(\mathrm{CR}_{0}^{1}\left(\mathcal{T}_{h}\right) / \mathbb{P}_{0}\left(\mathcal{T}_{h}\right)\right)$. This subsection is devoted to the a priori and a posteriori error analysis for the pair Crouzeix-Raviart finite element space $\left(\mathrm{CR}_{0}^{1}\left(\mathcal{T}_{h}\right)\right)$ for velocity and piecewise constant polynomial space $\mathbb{P}_{0}\left(\mathcal{T}_{h}\right)$ for pressure. 
4.2.1. Discrete distributed control problem: Consider the model problem from Subsection 3.1. Set

$$
X=\left[H_{0}^{1}(\Omega)\right]^{2}, W=\left[L^{2}(\Omega)\right]^{2}, M=L_{0}^{2}(\Omega) \text { and } Q=\left[L^{2}(\Omega)\right]^{2} .
$$

The set $Q_{a d}=Q_{d}$, where $Q_{d}$ is defined in (3.2). The discrete spaces are defined by

$$
X_{h}=\mathrm{CR}_{0}^{1}\left(\mathcal{T}_{h}\right), M_{h}:=\mathbb{P}_{0}\left(\mathcal{T}_{h}\right) \cap L_{0}^{2}(\Omega) \text { and } Q_{h}=\mathbb{P}_{0}\left(\mathcal{T}_{h}\right) .
$$

The admissible control set is $Q_{a d}^{h}=\left\{\mathbf{x}_{h} \in Q_{h}: \mathbf{y}_{a} \leq \mathbf{x}_{h} \leq \mathbf{y}_{b}\right\}$. It is clear that $Q_{a d}^{h} \subseteq Q_{a d}$ and $\Pi_{h} \mathbf{u} \in Q_{a d}^{h}$ for $\mathbf{u} \in Q_{a d}$. The operators $E: X \rightarrow Q$ and $E_{h}: X_{h} \rightarrow Q_{h}$ are inclusion maps. The bilinear forms for the diffusion term and pressure term are given by for all $\mathbf{v}_{h}, \mathbf{z}_{h} \in X_{h}$

$$
a_{h}\left(\mathbf{v}_{h}, \mathbf{z}_{h}\right)=\sum_{T \in \mathcal{T}_{h}} \int_{T} \nabla \mathbf{v}_{h}: \nabla \mathbf{z}_{h} \mathrm{dx}, \quad b_{h}\left(\mathbf{z}_{h}, p_{h}\right)=-\sum_{T \in \mathcal{T}_{h}} \int_{T} p_{h} \nabla \cdot \mathbf{z}_{h} \mathrm{dx} .
$$

Energy norm on $X_{h}$ is defined by

$$
\left\|\mathbf{v}_{h}\right\|_{h}^{2}=\sum_{T \in \mathcal{T}_{h}} \int_{T}\left|\nabla \mathbf{v}_{h}\right|^{2} \mathrm{dx}
$$

and $b_{h}(\cdot, \cdot)$ satisfies the inf-sup condition can be found in [15]. Assumptions (2.4) and (2.5) are the Poincaré-Friedrichs type inequality in [7, pp. 301].

Theorem 4.2. (Best approximation for velocity and control) It holds

$$
\begin{aligned}
\left\|\mathbf{y}-\mathbf{y}_{h}\right\|_{0, \Omega}+ & \left\|\boldsymbol{\phi}-\boldsymbol{\phi}_{h}\right\|_{h}+\left\|\mathbf{u}-\mathbf{u}_{h}\right\|_{h} \leq C\left(\inf _{\mathbf{w}_{h} \in Z_{h}}\left\|\mathbf{u}-\mathbf{w}_{h}\right\|_{h}+\inf _{\mathbf{w}_{h} \in Z_{h}}\left\|\boldsymbol{\phi}-\mathbf{w}_{h}\right\|_{h}\right. \\
& +\inf _{s_{h} \in M_{h}}\left\|p-s_{h}\right\|_{0, \Omega}+\inf _{s_{h} \in M_{h}}\left\|r-s_{h}\right\|_{0, \Omega}+h\|\mathbf{f}\|_{0, \Omega}+h \inf _{\mathbf{x}_{h} \in Q_{h}}\left\|\mathbf{y}-\mathbf{x}_{h}\right\|_{0, \Omega} \\
& \left.+h\left\|\mathbf{u}-\mathbf{u}_{d}\right\|_{0, \Omega}+\left\|\boldsymbol{\phi}-\Pi_{h} \boldsymbol{\phi}\right\|_{0, \Omega}+\left\|\mathbf{y}-\Pi_{h} \mathbf{y}\right\|_{0, \Omega}\right) .
\end{aligned}
$$

Proof. From Theorem 2.4 we know that

$$
\begin{gathered}
\left\|\boldsymbol{\phi}-\boldsymbol{\phi}_{h}\right\|_{h} \leq C\left(\left\|\boldsymbol{\phi}-\bar{P}_{h} \boldsymbol{\phi}\right\|_{h}+\left\|\boldsymbol{\phi}-\Pi_{h} \boldsymbol{\phi}\right\|_{0, \Omega}+\left\|\mathbf{y}-\Pi_{h} \mathbf{y}\right\|_{0, \Omega}+\left\|\mathbf{u}-P_{h} \mathbf{u}\right\|_{h}\right), \\
\left\|\mathbf{u}-\mathbf{u}_{h}\right\|_{h} \leq C\left(\left\|\mathbf{u}-P_{h} \mathbf{u}\right\|_{h}+\left\|\boldsymbol{\phi}-\Pi_{h} \phi\right\|_{0, \Omega}+\left\|\mathbf{y}-\Pi_{h} \mathbf{y}\right\|_{0, \Omega}+\left\|\boldsymbol{\phi}-\bar{P}_{h} \boldsymbol{\phi}\right\|_{h}\right) .
\end{gathered}
$$

Also from the best approximation results [4, Theorem 3.1], we have

$$
\left\|\boldsymbol{\phi}-\bar{P}_{h} \boldsymbol{\phi}\right\|_{h} \leq C\left(\inf _{\mathbf{w}_{h} \in Z_{h}}\left\|\boldsymbol{\phi}-\mathbf{w}_{h}\right\|_{h}+\inf _{s_{h} \in M_{h}}\left\|r-s_{h}\right\|_{0, \Omega}+h\left\|\mathbf{u}-\mathbf{u}_{d}\right\|_{0, \Omega}\right)
$$

and

$$
\left\|\mathbf{u}-P_{h} \mathbf{u}\right\|_{h} \leq C\left(\inf _{\mathbf{v}_{h} \in Z_{h}}\left\|\mathbf{u}-\mathbf{v}_{h}\right\|_{h}+\inf _{s_{h} \in M_{h}}\left\|p-s_{h}\right\|_{0, \Omega}+h\|\mathbf{f}\|_{0, \Omega}+h \inf _{\mathbf{x}_{h} \in Q_{h}}\left\|\mathbf{y}-\mathbf{x}_{h}\right\|_{0, \Omega}\right) .
$$

Theorem 2.3 shows that

$$
\left\|\mathbf{y}-\mathbf{y}_{h}\right\|_{0, \Omega} \leq C\left(\left\|\boldsymbol{\phi}-\Pi_{h} \boldsymbol{\phi}\right\|_{0, \Omega}+\left\|\mathbf{y}-\Pi_{h} \mathbf{y}\right\|_{0, \Omega}+\left\|\boldsymbol{\phi}-\bar{P}_{h} \boldsymbol{\phi}\right\|_{h}+\left\|\mathbf{u}-P_{h} \mathbf{u}\right\|_{h}\right) .
$$

Using the above estimates, we find

$$
\left\|\mathbf{y}-\mathbf{y}_{h}\right\|_{0, \Omega}+\left\|\boldsymbol{\phi}-\boldsymbol{\phi}_{h}\right\|_{h}+\left\|\mathbf{u}-\mathbf{u}_{h}\right\|_{h} \leq C\left(\inf _{\mathbf{w}_{h} \in Z_{h}}\left\|\mathbf{u}-\mathbf{w}_{h}\right\|_{h}+\inf _{\mathbf{w}_{h} \in Z_{h}}\left\|\boldsymbol{\phi}-\mathbf{w}_{h}\right\|_{h}\right.
$$




$$
\begin{aligned}
& +\inf _{s_{h} \in M_{h}}\left\|p-s_{h}\right\|_{0, \Omega}+\inf _{s_{h} \in M_{h}}\left\|r-s_{h}\right\|_{0, \Omega}+h\|\mathbf{f}\|_{0, \Omega}+h \inf _{\mathbf{x}_{h} \in Q_{h}}\left\|\mathbf{y}-\mathbf{x}_{h}\right\|_{0, \Omega} \\
& \left.+h\left\|\mathbf{u}-\mathbf{u}_{d}\right\|_{0, \Omega}+\left\|\boldsymbol{\phi}-\Pi_{h} \boldsymbol{\phi}\right\|_{0, \Omega}+\left\|\mathbf{y}-\Pi_{h} \mathbf{y}\right\|_{0, \Omega}\right) .
\end{aligned}
$$

This concludes the proof.

Theorem 4.3. (Best approximation for pressure) It holds

$$
\begin{aligned}
\left\|p-p_{h}\right\|_{0, \Omega} \leq C & \left(\inf _{\mathbf{v}_{h} \in Z_{h}}\left\|\mathbf{u}-\mathbf{v}_{h}\right\|_{h}+\inf _{\mathbf{w}_{h} \in Z_{h}}\left\|\boldsymbol{\phi}-\mathbf{w}_{h}\right\|_{h}+\inf _{s_{h} \in M_{h}}\left\|p-s_{h}\right\|_{0, \Omega}\right. \\
& +\inf _{s_{h} \in M_{h}}\left\|r-s_{h}\right\|_{0, \Omega}+h\|\mathbf{f}\|_{0, \Omega}+h \inf _{\mathbf{x}_{h} \in Q_{h}}\left\|\mathbf{y}-\mathbf{x}_{h}\right\|_{0, \Omega}+h\left\|\mathbf{u}-\mathbf{u}_{d}\right\|_{0, \Omega} \\
& \left.+\left\|p-P^{0} p\right\|_{0, \Omega}+\left\|\boldsymbol{\phi}-\Pi_{h} \boldsymbol{\phi}\right\|_{0, \Omega}+\left\|\mathbf{y}-\Pi_{h} \mathbf{y}\right\|_{0, \Omega}\right) .
\end{aligned}
$$

Proof. From Theorem 2.5, the pressure estimate is given by

$$
\begin{aligned}
\left\|p-p_{h}\right\|_{0, \Omega} \leq C\left(\left\|p-R_{h} p\right\|_{0, \Omega}+\left\|\mathbf{u}-P_{h} \mathbf{u}\right\|_{h}\right. & +\left\|\boldsymbol{\phi}-\bar{P}_{h} \boldsymbol{\phi}\right\|_{h} \\
& \left.+\left\|\boldsymbol{\phi}-\Pi_{h} \boldsymbol{\phi}\right\|_{0, \Omega}+\left\|\mathbf{y}-\Pi_{h} \mathbf{y}\right\|_{0, \Omega}\right) .
\end{aligned}
$$

From the best approximation result [4, Theorem 4.1] for pressure we have

$$
\begin{aligned}
\left\|p-R_{h} p\right\|_{0, \Omega} \leq C\left(\inf _{\mathbf{v}_{h} \in Z_{h}}\left\|\mathbf{u}-\mathbf{v}_{h}\right\|_{h}+\inf _{q_{h} \in M_{h}}\left\|p-q_{h}\right\|_{0, \Omega}\right. & +\left\|p-P^{0} p\right\|_{0, \Omega} \\
& \left.+h \inf _{\mathbf{x}_{h} \in Q_{h}}\left\|\mathbf{y}-\mathbf{x}_{h}\right\|_{0, \Omega}+h\|\mathbf{f}\|_{0, \Omega}\right),
\end{aligned}
$$

where $P^{0}$ is the $L^{2}$-projection onto the space of piecewise constant polynomials. A use the above estimates with (4.3) and (4.4) leads to (4.5). This concludes the proof.

Now we use elliptic regularity to derive concrete error estimates. Note that by wellposedness of the problem, $\mathbf{u}, \boldsymbol{\phi} \in\left[H_{0}^{1}(\Omega)\right]^{2}$ and $p \in L_{0}^{2}(\Omega)$. The elliptic regularity on polygonal domains implies that $\phi \in\left[H^{1+s}(\Omega) \cap H_{0}^{1}(\Omega)\right]^{2}$ and $r \in H^{s}(\Omega) \cap L_{0}^{2}(\Omega)$ for some $s \in(0,1]$, which depends on the interior angles of the domain $\Omega$. We know that, $\mathbf{y}=\Pi_{\left[\mathbf{y}_{a}, \mathbf{y}_{b}\right]}\left(-\frac{\phi}{\lambda}\right)$ on $\Omega$. Hence, the control $\mathbf{y} \in H^{1}(\Omega)$ and $\mathbf{u} \in\left[H^{1+s}(\Omega) \cap H_{0}^{1}(\Omega)\right]^{2}$ and $p \in H^{s}(\Omega) \cap L_{0}^{2}(\Omega)$. Using the Nédélec interpolation [4, Eq. 3.4], 30] we have the following estimates

$$
\inf _{\mathbf{v}_{h} \in Z_{h}}\left\|\mathbf{u}-\mathbf{v}_{h}\right\|_{h} \leq C h^{s}\|\mathbf{u}\|_{1+s} \text { and } \inf _{\mathbf{w}_{h} \in Z_{h}}\left\|\boldsymbol{\phi}-\mathbf{w}_{h}\right\|_{h} \leq C h^{s}\|\phi\|_{1+s}
$$

and

$$
\inf _{s_{h} \in M_{h}}\left\|p-s_{h}\right\|_{0, \Omega} \leq C h^{s}\|p\|_{s} \text { and } \inf _{s_{h} \in M_{h}}\left\|r-s_{h}\right\|_{0, \Omega} \leq C h^{s}\|r\|_{s} .
$$

Also we have the following estimates,

$$
\left\|\boldsymbol{\phi}-\Pi_{h} \boldsymbol{\phi}\right\|_{0, \Omega} \leq C h\|\boldsymbol{\phi}\|_{1+s, \Omega}, \text { and }\left\|\mathbf{y}-\Pi_{h} \mathbf{y}\right\|_{0, \Omega} \leq C h\|\mathbf{y}\|_{1, \Omega} .
$$

Substitution of the above approximation estimates in Theorem 4.2 and 4.3 results in

Theorem 4.4. Let $s \in(0,1]$ be the elliptic regularity index. Then, it holds

$$
\begin{array}{r}
\left\|\mathbf{y}-\mathbf{y}_{h}\right\|_{0, \Omega}+\left\|\boldsymbol{\phi}-\boldsymbol{\phi}_{h}\right\|_{h}+\left\|\mathbf{u}-\mathbf{u}_{h}\right\|_{h} \leq C\left(h^{s}\|\mathbf{u}\|_{1+s, \Omega}+h^{s}\|\boldsymbol{\phi}\|_{1+s, \Omega}+h^{s}\|p\|_{s, \Omega}+h^{s}\|r\|_{s, \Omega}\right. \\
\left.+h^{2}\|\mathbf{y}\|_{1, \Omega}+h\|\mathbf{f}\|_{0, \Omega}+h\left\|\mathbf{u}-\mathbf{u}_{d}\right\|_{0, \Omega}+h\|\boldsymbol{\phi}\|_{1+s, \Omega}+h\|\mathbf{y}\|_{1, \Omega}\right) .
\end{array}
$$


Theorem 4.5. Let $s \in(0,1]$ be the elliptic regularity index. Then there holds

$$
\begin{aligned}
\left\|p-p_{h}\right\|_{0, \Omega} \leq & C\left(h^{s}\|\mathbf{u}\|_{1+s, \Omega}+h^{s}\|\boldsymbol{\phi}\|_{1+s, \Omega}+h^{s}\|p\|_{s, \Omega}+h^{s}\|r\|_{s, \Omega}+h^{2}\|\mathbf{y}\|_{1, \Omega}\right. \\
& \left.+h\|\mathbf{f}\|_{0, \Omega}+h\left\|\mathbf{u}-\mathbf{u}_{d}\right\|_{0, \Omega}+h\|\boldsymbol{\phi}\|_{1+s, \Omega}+h\|\mathbf{y}\|_{1, \Omega}\right) .
\end{aligned}
$$

The following theorems deduce the reliable and efficient a posteriori error estimator.

Theorem 4.6. (A posteriori error estimator) It holds,

$$
\begin{aligned}
\left\|\mathbf{y}-\mathbf{y}_{h}\right\|_{0, \Omega}+\left\|\mathbf{u}-\mathbf{u}_{h}\right\|_{h}+\left\|p-p_{h}\right\|_{0, \Omega} & +\left\|\boldsymbol{\phi}-\boldsymbol{\phi}_{h}\right\|_{h}+\left\|r-r_{h}\right\|_{0, \Omega} \\
& \leq C\left(\eta_{(\mathbf{u}, p)}+\eta_{(\boldsymbol{\phi}, r)}+\left\|\boldsymbol{\phi}_{h}-\Pi_{h} \boldsymbol{\phi}_{h}\right\|_{0, \Omega}\right)
\end{aligned}
$$

where the estimators are defined as

$$
\eta_{(\mathbf{u}, p)}^{2}=\sum_{T \in \mathcal{T}_{h}}\left(h_{T}^{2}\left\|\mathbf{f}+\mathbf{y}_{h}\right\|_{0, T}^{2}\right)+\sum_{e \in \mathcal{E}_{h}^{i}} \| h^{\frac{1}{2}} \llbracket\left[p_{h} I-\nabla \mathbf{u}_{h} \rrbracket\left\|_{0, e}^{2}+\sum_{e \in \mathcal{E}_{h}}\right\| h^{-\frac{1}{2}} \underline{\llbracket \mathbf{u}_{h} \rrbracket} \|_{0, e}^{2},\right.
$$

and

$$
\eta_{(\boldsymbol{\phi}, r)}^{2}=\sum_{T \in \mathcal{T}_{h}}\left(h_{T}^{2}\left\|\mathbf{u}_{h}-\mathbf{u}_{d}\right\|_{0, T}^{2}\right)+\sum_{e \in \mathcal{E}_{h}^{i}}\left\|h^{\frac{1}{2}} \llbracket r_{h} I+\nabla \boldsymbol{\phi}_{h} \rrbracket\right\|_{0, e}^{2}+\sum_{e \in \mathcal{E}_{h}}\left\|h^{-\frac{1}{2}} \underline{\llbracket \boldsymbol{\phi}_{h} \rrbracket}\right\|_{0, e}^{2} .
$$

Proof. From Theorem 2.8, 2.9 and 2.10, we have

$$
\begin{array}{r}
\left\|\mathbf{y}-\mathbf{y}_{h}\right\|_{0, \Omega}+\left\|\mathbf{u}-\mathbf{u}_{h}\right\|_{h}+\left\|p-p_{h}\right\|_{0, \Omega}+\left\|\boldsymbol{\phi}-\boldsymbol{\phi}_{h}\right\|_{h}+\left\|r-r_{h}\right\|_{0, \Omega} \leq C\left(\left\|R \mathbf{u}-\mathbf{u}_{h}\right\|_{h}\right. \\
\left.+\left\|R_{0} p-p_{h}\right\|_{0, \Omega}+\left\|\phi_{h}-\bar{R} \boldsymbol{\phi}\right\|_{h}+\left\|\bar{R}_{0} r-r_{h}\right\|_{0, \Omega}+\left\|\boldsymbol{\phi}_{h}-\Pi_{h} \boldsymbol{\phi}_{h}\right\|_{0, \Omega}\right) .
\end{array}
$$

The a posteriori error analysis in [26, Theorem 3.1], [8, Section 7] gives the following error estimates:

$$
\begin{aligned}
& \left\|R \mathbf{u}-\mathbf{u}_{h}\right\|_{h}+\left\|R_{0} p-p_{h}\right\|_{0, \Omega} \leq C \eta_{(\mathbf{u}, p)}, \\
& \left\|\bar{R} \boldsymbol{\phi}-\boldsymbol{\phi}_{h}\right\|_{h}+\left\|\bar{R}_{0} r-r_{h}\right\|_{0, \Omega} \leq C \eta_{(\boldsymbol{\phi}, r)} .
\end{aligned}
$$

By substituting (4.11) and (4.12) in (4.10), we conclude the proof.

Theorem 4.7. (Efficiency) Let $\mathcal{T}_{e}$ be the set of two triangles sharing the edge $e \in \mathcal{E}_{h}^{i}$. Then, It hold

$$
\begin{aligned}
h_{T}\left\|\mathbf{f}+\mathbf{y}_{h}\right\|_{0, T} & \leq C\left(\left\|\nabla\left(\mathbf{u}-\mathbf{u}_{h}\right)\right\|_{0, T}+\left\|p-p_{h}\right\|_{0, T}+\operatorname{osc}(\mathbf{f}, T)+\left\|\mathbf{y}-\mathbf{y}_{h}\right\|_{0, T}\right), \\
h_{T}\left\|\mathbf{u}_{h}-\mathbf{u}_{d}\right\|_{0, T} & \leq C\left(\left\|\mathbf{u}-\mathbf{u}_{h}\right\|_{0, T}+\left\|\nabla\left(\boldsymbol{\phi}-\boldsymbol{\phi}_{h}\right)\right\|_{0, T}+\left\|r-r_{h}\right\|_{0, T}+\operatorname{osc}\left(\mathbf{u}_{d}, T\right)\right), \\
\left.\| h^{\frac{1}{2}} \llbracket p_{h} I-\nabla \mathbf{u}_{h}\right] \|_{0, e} & \leq C \sum_{T \in \mathcal{T}_{e}}\left(\left\|\nabla\left(\mathbf{u}-\mathbf{u}_{h}\right)\right\|_{0, T}+\left\|p-p_{h}\right\|_{0, T}+\operatorname{osc}(\mathbf{f}, T)+\left\|\mathbf{y}-\mathbf{y}_{h}\right\|_{0, T}\right), \\
\left.\| h^{\frac{1}{2}} \llbracket r_{h} I+\nabla \boldsymbol{\phi}_{h}\right] \|_{0, e} & \leq C \sum_{T \in \mathcal{T}_{e}}\left(\left\|\mathbf{u}-\mathbf{u}_{h}\right\|_{0, T}+\left\|\nabla\left(\boldsymbol{\phi}-\boldsymbol{\phi}_{h}\right)\right\|_{0, T}+\left\|r-r_{h}\right\|_{0, T}+\operatorname{osc}\left(\mathbf{u}_{d}, T\right)\right), \\
\left\|\boldsymbol{\phi}_{h}-\Pi_{h} \boldsymbol{\phi}_{h}\right\|_{0, T} & \leq C\left(\left\|\boldsymbol{\phi}_{h}-\boldsymbol{\phi}\right\|_{0, T}+\left\|\boldsymbol{\phi}-\Pi_{h} \boldsymbol{\phi}_{h}\right\|_{0, T}\right) .
\end{aligned}
$$

Proof. The local efficiency can be deduced by the standard bubble function techniques [35]. 
4.2.2. Discrete boundary control problem: The model problem in this section is the Neumann boundary control problem introduced in Subsection 3.2. Set

$$
X=\left[H^{1}(\Omega) \cap L_{0}^{2}(\Omega)\right]^{2}, W=\left[L^{2}(\Omega)\right]^{2}, M=L^{2}(\Omega) \text { and } Q=\left[L^{2}(\Gamma)\right]^{2} .
$$

The continuous admissible control set is $Q_{b}$, where $Q_{b}$ is defined in (3.12). The discrete spaces are

$$
X_{h}=\mathrm{CR}^{1}\left(\mathcal{T}_{h}\right) \cap\left[L_{0}^{2}(\Omega)\right]^{2} \text { and } M_{h}=\mathbb{P}_{0}\left(\mathcal{T}_{h}\right) .
$$

Define discrete control space as

$$
Q_{h}=\left\{\mathbf{x}_{h} \in\left[L^{2}(\Gamma)\right]^{2}:\left.\mathbf{x}_{h}\right|_{e} \in\left[\mathbb{P}_{0}(e)\right]^{2} \text { for all } e \in \mathcal{E}_{h}^{b}\right\}
$$

and the admissible discrete control set is

$$
Q_{a d}^{h}=\left\{\mathbf{x}_{h} \in Q_{h}: \int_{\Gamma} \mathbf{x}_{\mathbf{h}} \mathrm{ds}+\int_{\Omega} \mathbf{f} \mathrm{dx}=\mathbf{0}, \quad \mathbf{y}_{a} \leq \mathbf{x}_{h} \leq \mathbf{y}_{b}\right\}
$$

It is clear that $Q_{a d}^{h} \subseteq Q_{a d}$ and $\Pi_{h} \mathbf{u} \in Q_{a d}^{h}$ for $\mathbf{u} \in Q_{a d}$. The operator $E: X \rightarrow Q$ is the trace map and $E_{h}: X_{h} \rightarrow Q_{h}$ is defined by the piecewise (edge-wise) trace, i.e., $\left.E_{h} \mathbf{w}\right|_{e}=\left.\mathbf{w}_{T}\right|_{e}$ where $\mathbf{w}_{T}=\left.\mathbf{w}\right|_{T}$ and $T$ be the triangle having the edge $e$ on boundary. The bilinear forms are given by

$$
a_{h}\left(\mathbf{v}_{h}, \mathbf{z}_{h}\right)=\sum_{T \in \mathcal{T}_{h}} \int_{T} \nabla \mathbf{v}_{h}: \nabla \mathbf{z}_{h} \mathrm{dx}, b_{h}\left(\mathbf{z}_{h}, p_{h}\right)=-\sum_{T \in \mathcal{T}_{h}} \int_{T} p_{h} \nabla \cdot \mathbf{z}_{h} \mathrm{dx}
$$

for all $\mathbf{v}_{h}, \mathbf{z}_{h} \in X_{h}$ and $p_{h} \in M_{h}$. The energy norm on $X_{h}$ is defined by

$$
\left\|\mathbf{v}_{h}\right\|_{h}^{2}=\sum_{T \in \mathcal{T}_{h}} \int_{T}\left|\nabla \mathbf{v}_{h}\right|^{2} \mathrm{dx} .
$$

The inequality (2.4) follows from the results Poincaré-Friedrichs type inequalities in [6]. The estimate in (2.5) follows from the well-known trace inequality and [6].

Theorem 4.8. Let $s \in(0,1]$ be the elliptic regularity index. Then, it holds

$$
\begin{array}{r}
\left\|\mathbf{y}-\mathbf{y}_{h}\right\|_{0, \Gamma}+\left\|\boldsymbol{\phi}-\boldsymbol{\phi}_{h}\right\|_{h}+\left\|\mathbf{u}-\mathbf{u}_{h}\right\|_{h} \leq C\left(h^{s}\|\mathbf{u}\|_{1+s, \Omega}+h^{s}\|\boldsymbol{\phi}\|_{1+s, \Omega}+h^{s}\|p\|_{s, \Omega}+h^{s}\|r\|_{s, \Omega}\right. \\
\left.+\sum_{i=1}^{m} h^{1+s}\|\mathbf{y}\|_{\frac{1}{2}+s, \Gamma_{i}}+h\|\mathbf{f}\|_{0, \Omega}+h\left\|\mathbf{u}-\mathbf{u}_{d}\right\|_{0, \Omega}+h\|\boldsymbol{\phi}\|_{1+s, \Omega}+\sum_{i=1}^{m} h^{\frac{1}{2}+s}\|\mathbf{y}\|_{\frac{1}{2}+s, \Gamma_{i}}\right) .
\end{array}
$$

Proof. From Theorem 2.4 we have

$$
\left\|\boldsymbol{\phi}-\boldsymbol{\phi}_{h}\right\|_{h} \leq C\left(\left\|\boldsymbol{\phi}-\bar{P}_{h} \boldsymbol{\phi}\right\|_{h}+\left\|\boldsymbol{\phi}-\Pi_{h} \boldsymbol{\phi}\right\|_{0, \Gamma}+\left\|\mathbf{y}-\Pi_{h} \mathbf{y}\right\|_{0, \Gamma}+\left\|\mathbf{u}-P_{h} \mathbf{u}\right\|_{h}\right),
$$

and

$$
\left\|\mathbf{u}-\mathbf{u}_{h}\right\|_{h} \leq C\left(\left\|\mathbf{u}-P_{h} \mathbf{u}\right\|_{h}+\left\|\boldsymbol{\phi}-\Pi_{h} \boldsymbol{\phi}\right\|_{0, \Gamma}+\left\|\mathbf{y}-\Pi_{h} \mathbf{y}\right\|_{0, \Gamma}+\left\|\boldsymbol{\phi}-\bar{P}_{h} \boldsymbol{\phi}\right\|_{h}\right) .
$$

As a consequence of the error analysis in [4, Theorem 3.1] for Neumann boundary problem we have,

$$
\left\|\boldsymbol{\phi}-\bar{P}_{h} \boldsymbol{\phi}\right\|_{h} \leq C\left(\inf _{\mathbf{w}_{h} \in Z_{h}}\left\|\boldsymbol{\phi}-\mathbf{w}_{h}\right\|_{h}+\inf _{s_{h} \in M_{h}}\left\|r-s_{h}\right\|_{0, \Omega}+h\left\|\mathbf{u}-\mathbf{u}_{d}\right\|_{0, \Omega}\right)
$$




$$
\left\|\mathbf{u}-\bar{P}_{h} \mathbf{u}\right\|_{h} \leq C\left(\inf _{\mathbf{v}_{h} \in Z_{h}}\left\|\mathbf{u}-\mathbf{v}_{h}\right\|_{h}+\inf _{r_{h} \in M_{h}}\left\|p-r_{h}\right\|_{0, \Omega}+h\|\mathbf{f}\|_{0, \Omega}+h^{\frac{1}{2}} \inf _{\mathbf{x}_{h} \in Q_{h}}\left\|\mathbf{y}-\mathbf{x}_{h}\right\|_{0, \Gamma}\right) .
$$

From the Theorem 2.3 we have

$$
\left\|\mathbf{y}-\mathbf{y}_{h}\right\|_{0, \Gamma} \leq C\left(\left\|\boldsymbol{\phi}-\Pi_{h} \boldsymbol{\phi}\right\|_{0, \Gamma}+\left\|\mathbf{y}-\Pi_{h} \mathbf{y}\right\|_{0, \Gamma}+\left\|\boldsymbol{\phi}-\bar{P}_{h} \boldsymbol{\phi}\right\|_{h}+\left\|\mathbf{u}-P_{h} \mathbf{u}\right\|_{h}\right) .
$$

Using the above estimates, we find

$$
\begin{aligned}
\left\|\mathbf{y}-\mathbf{y}_{h}\right\|_{0, \Gamma}+\left\|\boldsymbol{\phi}-\boldsymbol{\phi}_{h}\right\|_{h}+ & \left\|\mathbf{u}-\mathbf{u}_{h}\right\|_{h} \leq C\left(\inf _{\mathbf{v}_{h} \in Z_{h}}\left\|\mathbf{u}-\mathbf{v}_{h}\right\|_{h}+\inf _{\mathbf{w}_{h} \in Z_{h}}\left\|\boldsymbol{\phi}-\mathbf{w}_{h}\right\|_{h}\right. \\
& +\inf _{s_{h} \in M_{h}}\left\|p-s_{h}\right\|_{0, \Omega}+\inf _{s_{h} \in M_{h}}\left\|r-s_{h}\right\|_{0, \Omega}+h^{\frac{1}{2}} \inf _{\mathbf{x}_{h} \in Q_{h}}\left\|\mathbf{y}-\mathbf{x}_{h}\right\|_{0, \Gamma} \\
& \left.+h\|\mathbf{f}\|_{0, \Omega}+h\left\|\mathbf{u}-\mathbf{u}_{d}\right\|_{0, \Omega}+\left\|\boldsymbol{\phi}-\Pi_{h}(\boldsymbol{\phi})\right\|_{0, \Gamma}+\left\|\mathbf{y}-\Pi_{h} \mathbf{y}\right\|_{0, \Gamma}\right) .
\end{aligned}
$$

The elliptic regularity on polygonal domains implies that $\phi \in\left[H^{1+s}(\Omega)\right]^{2} \cap X$ and $r \in H^{s}(\Omega)$ for some $s \in(0,1]$, which depends on the interior angles of the domain $\Omega$. We know that, $\mathbf{y}=\Pi_{\left[\mathbf{y}_{a}, \mathbf{y}_{b}\right]}\left(-\frac{\phi}{\lambda}\right) \quad$ on $\partial \Omega$. Hence the control $\left.\mathbf{y}\right|_{\Gamma_{i}} \in\left[H^{\frac{1}{2}+s}\left(\Gamma_{i}\right)\right]^{2}$ for $1 \leq i \leq m$ and $\mathbf{u} \in\left[H^{1+s}(\Omega)\right]^{2} \cap X$ and $p \in H^{s}(\Omega)$, where $m$ is the number of boundary edges. Also we have the following estimates,

$$
\left\|\boldsymbol{\phi}-\Pi_{h}(\boldsymbol{\phi})\right\|_{0, \Gamma} \leq C h^{\frac{1}{2}+s}\|\boldsymbol{\phi}\|_{1+s, \Omega}, \text { and }\left\|\mathbf{y}-\Pi_{h}(\mathbf{y})\right\|_{0, \Gamma} \leq C \sum_{i=1}^{m} h^{\frac{1}{2}+s}\|\mathbf{y}\|_{\frac{1}{2}+s, \Gamma_{i}} .
$$

Using (4.6)-(4.8) and (4.17) in (4.16), we have proved the theorem.

Theorem 4.9. Let $s \in(0,1]$ be the elliptic regularity index. Then there holds

$$
\begin{aligned}
\left\|p-p_{h}\right\|_{0, \Omega} \leq & C\left(h^{s}\|\mathbf{u}\|_{1+s, \Omega}+h^{s}\|\boldsymbol{\phi}\|_{1+s, \Omega}+h^{s}\|p\|_{s, \Omega}+h^{s}\|r\|_{s, \Omega}+\sum_{i=1}^{m} h^{1+s}\|\mathbf{y}\|_{\frac{1}{2}+s, \Gamma_{i}}\right. \\
& \left.+h\|\mathbf{f}\|_{0, \Omega}+h\left\|\mathbf{u}-\mathbf{u}_{d}\right\|_{0, \Omega}+h\|\boldsymbol{\phi}\|_{1+s, \Omega}+\sum_{i=1}^{m} h^{\frac{1}{2}+s}\|\mathbf{y}\|_{\frac{1}{2}+s, \Gamma_{i}}\right) .
\end{aligned}
$$

Proof. From Theorem 2.5, we have the pressure estimate as below

$$
\begin{aligned}
\left\|p-p_{h}\right\|_{0, \Omega} \leq C\left(\left\|p-R_{h} p\right\|_{0, \Gamma}+\left\|\mathbf{u}-P_{h} \mathbf{u}\right\|_{h}+\left\|\boldsymbol{\phi}-\bar{P}_{h} \boldsymbol{\phi}\right\|_{h}\right. \\
\left.+\left\|\boldsymbol{\phi}-\Pi_{h} \boldsymbol{\phi}\right\|_{0, \Gamma}+\left\|\mathbf{y}-\Pi_{h} \mathbf{y}\right\|_{0, \Gamma}\right) .
\end{aligned}
$$

As a consequence of the error analysis in [4, Theorem 4.1] for Neumann boundary value problem, we have

$$
\begin{aligned}
\left\|p-R_{h} p\right\|_{0, \Omega} \leq & C\left(\inf _{\mathbf{v}_{h} \in Z_{h}}\left\|\mathbf{u}-\mathbf{v}_{h}\right\|_{h}+\inf _{q_{h} \in M_{h}}\left\|p-q_{h}\right\|_{0, \Omega}+\left\|p-P^{0} p\right\|_{0, \Omega}\right. \\
& \left.+h^{\frac{1}{2}} \inf _{\mathbf{x}_{h} \in Q_{h}}\left\|\mathbf{y}-\mathbf{x}_{h}\right\|_{0, \Gamma}+h\|\mathbf{f}\|_{0, \Omega}\right) .
\end{aligned}
$$

Using the above estimates and (4.14)-(4.15) we get

$$
\left\|p-p_{h}\right\|_{0, \Omega} \leq C\left(\inf _{\mathbf{v}_{h} \in Z_{h}}\left\|\mathbf{u}-\mathbf{v}_{h}\right\|_{h}+\inf _{\mathbf{w}_{h} \in Z_{h}}\left\|\boldsymbol{\phi}-\mathbf{w}_{h}\right\|_{h}+\inf _{s_{h} \in M_{h}}\left\|p-s_{h}\right\|_{0, \Omega}\right.
$$




$$
\begin{aligned}
& +\inf _{s_{h} \in M_{h}}\left\|r-s_{h}\right\|_{0, \Omega}+h\|\mathbf{f}\|_{0, \Omega}+h^{\frac{1}{2}} \inf _{\mathbf{x}_{h} \in Q_{h}}\left\|\mathbf{y}-\mathbf{x}_{h}\right\|_{0, \Gamma}+h\left\|\mathbf{u}-\mathbf{u}_{d}\right\|_{0, \Omega} \\
& \left.+\left\|p-P^{0} p\right\|_{0, \Omega}+\left\|\boldsymbol{\phi}-\Pi_{h} \boldsymbol{\phi}\right\|_{0, \Gamma}+\left\|\mathbf{y}-\Pi_{h} \mathbf{y}\right\|_{0, \Gamma}\right)
\end{aligned}
$$

Finally, use (4.6), (4.7) and (4.8) in above estimate concludes the proof.

Theorem 4.10. (A posteriori error estimator) It holds,

$$
\begin{aligned}
\left\|\mathbf{y}-\mathbf{y}_{h}\right\|_{0, \Gamma}+\left\|\mathbf{u}-\mathbf{u}_{h}\right\|_{h} & +\left\|p-p_{h}\right\|_{0, \Omega}+\left\|\boldsymbol{\phi}-\boldsymbol{\phi}_{h}\right\|_{h}+\left\|r-r_{h}\right\|_{0, \Omega} \\
& \leq C\left(\eta_{(\mathbf{u}, p)}+\eta_{(\phi, r)}+\left\|\phi_{h}-\Pi_{h} \boldsymbol{\phi}_{h}\right\|_{0, \Gamma}\right)
\end{aligned}
$$

where the estimators are defined as

$$
\begin{aligned}
& \eta_{(\mathbf{u}, p)}^{2}=\sum_{T \in \mathcal{T}_{h}} h_{T}^{2}\|\mathbf{f}\|_{0, T}^{2}+\sum_{e \in \mathcal{E}_{h}^{\text {int }}} \| h^{\frac{1}{2}} \llbracket\left[p_{h} I-\nabla \mathbf{u}_{h} \rrbracket \|_{0, e}^{2}\right. \\
& +\sum_{e \in \mathcal{E}_{h}^{b}}\left\|h^{\frac{1}{2}}\left(p_{h} I-\nabla \mathbf{u}_{h}+\mathbf{y}_{h} I\right)\right\|_{0, e}^{2}+\sum_{e \in \mathcal{E}_{h}^{i}}\left\|h^{-\frac{1}{2}} \underline{\llbracket \mathbf{u}_{h} \rrbracket}\right\|_{0, e}^{2},
\end{aligned}
$$

and

$$
\begin{aligned}
\eta_{(\boldsymbol{\phi}, r)}^{2}=\sum_{T \in \mathcal{T}_{h}} h_{T}^{2}\left\|\mathbf{u}_{h}-\mathbf{u}_{d}\right\|_{0, T}^{2} & +\sum_{e \in \mathcal{E}_{h}^{i n t}}\left\|h^{\frac{1}{2}} \llbracket r_{h} I+\nabla \boldsymbol{\phi}_{h} \rrbracket\right\|_{0, e}^{2} \\
& +\sum_{e \in \mathcal{E}_{h}^{b}}\left\|h^{\frac{1}{2}} \llbracket r_{h} I+\nabla \boldsymbol{\phi}_{h} \rrbracket\right\|_{0, e}^{2}+\sum_{e \in \mathcal{E}_{h}^{i}}\left\|h^{-\frac{1}{2}} \underline{\llbracket \boldsymbol{\phi}_{h} \rrbracket}\right\|_{0, e}^{2} .
\end{aligned}
$$

Proof. From Theorem 2.8, 2.9] and 2.10, we have

$$
\begin{array}{r}
\left\|\mathbf{y}-\mathbf{y}_{h}\right\|_{0, \Gamma}+\left\|\mathbf{u}-\mathbf{u}_{h}\right\|_{h}+\left\|p-p_{h}\right\|_{0, \Omega}+\left\|\boldsymbol{\phi}-\boldsymbol{\phi}_{h}\right\|_{h}+\left\|r-r_{h}\right\|_{0, \Omega} \leq C\left(\left\|R \mathbf{u}-\mathbf{u}_{h}\right\|_{h}\right. \\
\left.+\left\|R_{0} p-p_{h}\right\|_{0, \Omega}+\left\|\boldsymbol{\phi}_{h}-\bar{R} \boldsymbol{\phi}\right\|_{h}+\left\|\bar{R}_{0} r-r_{h}\right\|_{0, \Omega}+\left\|\boldsymbol{\phi}_{h}-\Pi_{h} \boldsymbol{\phi}_{h}\right\|_{0, \Gamma}\right) .
\end{array}
$$

As a consequence of the error analysis in [26, Theorem 3.1] for Neumann boundary value problem, concludes the following error estimates:

$$
\begin{gathered}
\left\|R \mathbf{u}-\mathbf{u}_{h}\right\|_{h}+\left\|R_{0} p-p_{h}\right\|_{0, \Omega} \leq C \eta_{(\mathbf{u}, p)}, \\
\left\|\bar{R} \boldsymbol{\phi}-\boldsymbol{\phi}_{h}\right\|_{h}+\left\|\bar{R}_{0} r-r_{h}\right\|_{0, \Omega} \leq C \eta_{(\boldsymbol{\phi}, r)} .
\end{gathered}
$$

The substitution of (4.20) and (4.21) in (4.19) completes the proof of (4.18).

Theorem 4.11. (Efficiency) Let $\mathcal{T}_{e}$ be the set of two triangles sharing the edge $e \in \mathcal{E}_{h}^{i}$. Then, it hold

$$
\begin{aligned}
h_{T}\|\mathbf{f}\|_{0, T} & \leq C\left(\left\|\nabla\left(\mathbf{u}-\mathbf{u}_{h}\right)\right\|_{0, T}+\left\|p-p_{h}\right\|_{0, T}+\operatorname{osc}(\mathbf{f}, T)\right), \\
h_{T}\left\|\mathbf{u}_{h}-\mathbf{u}_{d}\right\|_{0, T} & \leq C\left(\left\|\mathbf{u}-\mathbf{u}_{h}\right\|_{0, T}+\left\|\nabla\left(\boldsymbol{\phi}-\boldsymbol{\phi}_{h}\right)\right\|_{0, T}+\left\|r-r_{h}\right\|_{0, T}+\operatorname{osc}\left(\mathbf{u}_{d}, T\right)\right), \\
\left\|h^{\frac{1}{2}}\left[p_{h} I-\nabla \mathbf{u}_{h}\right]\right\|_{0, e} & \leq C \sum_{T \in \mathcal{T}_{e}}\left(\left\|\nabla\left(\mathbf{u}-\mathbf{u}_{h}\right)\right\|_{0, T}+\left\|p-p_{h}\right\|_{0, T}+\operatorname{osc}(\mathbf{f}, T)+\left\|\mathbf{y}-\mathbf{y}_{h}\right\|_{0, T}\right),
\end{aligned}
$$




$$
\begin{aligned}
\left\|h^{\frac{1}{2}}\left[r_{h} I+\nabla \phi_{h}\right]\right\|_{0, e} \leq C \sum_{T \in \mathcal{T}_{e}}\left(\left\|\mathbf{u}-\mathbf{u}_{h}\right\|_{0, T}+\left\|\nabla\left(\boldsymbol{\phi}-\boldsymbol{\phi}_{h}\right)\right\|_{0, T}+\left\|r-r_{h}\right\|_{0, T}+\operatorname{osc}\left(\mathbf{u}_{d}, T\right)\right), \\
\left\|\boldsymbol{\phi}_{h}-\Pi_{h} \boldsymbol{\phi}_{h}\right\|_{0, T} \leq C\left(\left\|\boldsymbol{\phi}_{h}-\boldsymbol{\phi}\right\|_{0, T}+\left\|\boldsymbol{\phi}-\Pi_{h} \boldsymbol{\phi}_{h}\right\|_{0, T}\right) .
\end{aligned}
$$

Further, for any boundary edge $e \in \mathcal{E}_{h}^{b}$, it hold

$$
\begin{aligned}
\left\|h^{\frac{1}{2}}\left(p_{h} I-\nabla \mathbf{u}_{h}+\mathbf{y}_{h} I\right)\right\|_{0, e} & \leq C\left(\left\|\nabla\left(\mathbf{u}-\mathbf{u}_{h}\right)\right\|_{0, T}+\left\|p-p_{h}\right\|_{0, T}+o s c(\mathbf{f}, T)+\left\|\mathbf{y}-\mathbf{y}_{h}\right\|_{0, T}\right), \\
\left\|h^{\frac{1}{2}}\left(r_{h} I+\nabla \phi_{h}\right)\right\|_{0, e} & \leq C\left(\left\|\mathbf{u}-\mathbf{u}_{h}\right\|_{0, T}+\left\|\nabla\left(\boldsymbol{\phi}-\phi_{h}\right)\right\|_{0, T}+\left\|r-r_{h}\right\|_{0, T}+\operatorname{osc}\left(\mathbf{u}_{d}, T\right)\right)
\end{aligned}
$$

Proof. The above theorem on local efficiency can be deduced by the standard bubble functions technique [35].

4.3. Discontinuous Galerkin Method $\mathbb{P}_{1}\left(\mathcal{T}_{h}\right) / \mathbb{P}_{0}\left(\mathcal{T}_{h}\right)$. In this subsection, we will discuss about discrete problem for $\mathrm{DG}$ pair $\mathbb{P}_{1} / \mathbb{P}_{0}$ of velocity and pressure, respectively.

\subsubsection{Discrete distributed control problem: Set}

$$
X=\left[H_{0}^{1}(\Omega)\right]^{2}, W=\left[L^{2}(\Omega)\right]^{2}, M=L_{0}^{2}(\Omega) \text { and } Q=\left[L^{2}(\Omega)\right]^{2} .
$$

The set $Q_{a d}=Q_{d}$, where $Q_{d}$ is defined in (3.2). The discrete spaces are defined by

$$
X_{h}:=\left[\mathbb{P}_{1}\left(\mathcal{T}_{h}\right)\right]^{2} \text {, and } M_{h}:=\mathbb{P}_{0}\left(\mathcal{T}_{h}\right) \cap L_{0}^{2}(\Omega) .
$$

The admissible control set $Q_{a d}^{h}=\left\{\mathbf{x}_{h} \in\left[\mathbb{P}_{0}\left(\mathcal{T}_{h}\right)\right]^{2}: \mathbf{y}_{a} \leq \mathbf{x}_{h} \leq \mathbf{y}_{b}\right\}$. It is clear that $Q_{a d}^{h} \subseteq Q_{a d}$ and $\Pi_{h} \mathbf{u} \in Q_{a d}^{h}$ for $\mathbf{u} \in Q_{a d}$. The operators $E: X \rightarrow Q$ and $E_{h}: X_{h} \rightarrow Q_{h}$ are inclusion maps.

The interior penalty DG bilinear form for the diffusion term is given by

$$
\begin{aligned}
a_{h}\left(\mathbf{v}_{h}, \mathbf{z}_{h}\right)= & \sum_{T \in \mathcal{T}_{h}} \int_{T} \nabla \mathbf{v}_{h}: \nabla \mathbf{z}_{h} \mathrm{dx}-\sum_{e \in \mathcal{E}_{h}} \int_{e}\left\{\left\{\nabla \mathbf{v}_{h}\right\}\right\}: \underline{\llbracket \mathbf{z}_{h} \rrbracket} \mathrm{ds}-\sum_{e \in \mathcal{E}_{h}} \int_{e}\left\{\left\{\nabla \mathbf{z}_{h}\right\}\right\}: \underline{\llbracket \mathbf{v}_{h} \rrbracket} \mathrm{ds} \\
& +\sum_{e \in \mathcal{E}_{h}} \frac{\sigma}{h_{e}} \int_{e} \underline{\llbracket \mathbf{v}_{h} \rrbracket}: \underline{\left.\llbracket \mathbf{z}_{h} \rrbracket\right]} \mathrm{ds},
\end{aligned}
$$

for all $\mathbf{v}_{h}, \mathbf{z}_{h} \in X_{h}$ and $\sigma>0$ be a real number. The DG bilinear form for the pressure term is given by

$$
b_{h}\left(\mathbf{z}_{h}, p_{h}\right)=-\sum_{T \in \mathcal{T}_{h}} \int_{T} p_{h} \nabla \cdot \mathbf{z}_{h} \mathrm{dx}+\sum_{e \in \mathcal{E}_{h}} \int_{e}\left\{\left\{p_{h}\right\}\right\}\left[\mathbf{z}_{h}\right] \mathrm{ds},
$$

for all $\mathbf{z}_{h} \in X_{h}$ and $p_{h} \in M_{h}$. After an integration by parts on the right-hand side of (4.23), we have

$$
b_{h}\left(\mathbf{z}_{h}, p_{h}\right)=\sum_{T \in \mathcal{T}_{h}} \int_{T} \mathbf{z}_{h} \cdot \nabla p_{h} \mathrm{dx}-\sum_{e \in \mathcal{E}_{h}^{i}} \int_{e}\left\{\left\{p_{h}\right\}\right\} \llbracket\left[\mathbf{z}_{h} \rrbracket \mathrm{ds},\right.
$$


for all $\mathbf{z}_{h} \in X_{h}$ and $p_{h} \in M_{h}$. We choose $\sigma>0$ large enough such that $a_{h}(\cdot, \cdot)$ is $X_{h}$-elliptic with respect to the norm $\|\cdot\|_{h}$ on $X_{h}$ which is given by

$$
\left\|\mathbf{v}_{h}\right\|_{h}^{2}=\sum_{T \in \mathcal{T}_{h}} \int_{T}\left|\nabla \mathbf{v}_{h}\right|^{2} \mathrm{dx}+\sum_{e \in \mathcal{E}_{h}} \frac{1}{h_{e}} \int_{e} \underline{\left[\mathbf{v}_{h} \rrbracket^{2}\right.} \mathrm{ds}
$$

and the fact that $b_{h}(\cdot, \cdot)$ satisfies the inf-sup condition can be found in [15]. Assumptions (2.4) and (2.5) are the Poincaré type inequalities derived in [6].

Theorem 4.12. Let $s \in(0,1]$ be the elliptic regularity index. Then it holds

$$
\begin{aligned}
\left\|\mathbf{y}-\mathbf{y}_{h}\right\|_{0, \Omega}+ & \left\|\boldsymbol{\phi}-\boldsymbol{\phi}_{h}\right\|_{h}+\left\|\mathbf{u}-\mathbf{u}_{h}\right\|_{h} \leq C\left(h^{s}\|\mathbf{u}\|_{1+s, \Omega}+h^{s}\|\boldsymbol{\phi}\|_{1+s, \Omega}+h^{s}\|p\|_{s, \Omega}+h^{s}\|r\|_{s, \Omega}\right. \\
+ & \left.+h \inf _{\mathbf{x}_{h} \in Q_{h}}\left\|\mathbf{y}-\mathbf{x}_{h}\right\|_{0, \Omega}+h\|\mathbf{f}\|_{0, \Omega}+h\left\|\mathbf{u}-\mathbf{u}_{d}\right\|_{0, \Omega}+h\|\boldsymbol{\phi}\|_{1+s, \Omega}+h\|\mathbf{y}\|_{1, \Omega}\right) .
\end{aligned}
$$

Proof. The best approximation results [4, Theorem 3.1], give

$$
\left\|\boldsymbol{\phi}-\bar{P}_{h} \boldsymbol{\phi}\right\|_{h} \leq C\left(\inf _{\mathbf{w}_{h} \in Z_{h}}\left\|\boldsymbol{\phi}-\mathbf{w}_{h}\right\|_{h}+\inf _{s_{h} \in M_{h}}\left\|r-s_{h}\right\|_{0, \Omega}+h\left\|\mathbf{u}-\mathbf{u}_{d}\right\|_{0, \Omega}\right)
$$

and

$$
\left\|\mathbf{u}-\bar{P}_{h} \mathbf{u}\right\|_{h} \leq C\left(\inf _{\mathbf{v}_{h} \in Z_{h}}\left\|\mathbf{u}-\mathbf{v}_{h}\right\|_{h}+\inf _{s_{h} \in M_{h}}\left\|p-s_{h}\right\|_{0, \Omega}+h\|\mathbf{f}\|_{0, \Omega}+h \inf _{\mathbf{x}_{h} \in Q_{h}}\left\|\mathbf{y}-\mathbf{x}_{h}\right\|_{0, \Omega}\right) .
$$

From Theorem 2.3 we get

$$
\left\|\mathbf{y}-\mathbf{y}_{h}\right\|_{0, \Omega} \leq C\left(\left\|\boldsymbol{\phi}-\Pi_{h}(\boldsymbol{\phi})\right\|_{0, \Omega}+\left\|\mathbf{y}-\Pi_{h} \mathbf{y}\right\|_{0, \Omega}+\left\|\boldsymbol{\phi}-\bar{P}_{h} \boldsymbol{\phi}\right\|_{h}+\left\|\mathbf{u}-P_{h} \mathbf{u}\right\|_{h}\right) .
$$

A use of the above estimates in (2.23) from Theorem 2.4 results in

$$
\begin{aligned}
\left\|\mathbf{y}-\mathbf{y}_{h}\right\|_{0, \Omega}+ & \left\|\boldsymbol{\phi}-\boldsymbol{\phi}_{h}\right\|_{h}+\left\|\mathbf{u}-\mathbf{u}_{h}\right\|_{h} \leq C\left(\inf _{\mathbf{v}_{h} \in Z_{h}}\left\|\mathbf{u}-\mathbf{v}_{h}\right\|_{h}+\inf _{\mathbf{w}_{h} \in Z_{h}}\left\|\boldsymbol{\phi}-\mathbf{w}_{h}\right\|_{h}\right. \\
& +\inf _{s_{h} \in M_{h}}\left\|p-s_{h}\right\|_{0, \Omega}+\inf _{s_{h} \in M_{h}}\left\|r-s_{h}\right\|_{0, \Omega}+h\|\mathbf{f}\|_{0, \Omega}+h \inf _{\mathbf{x}_{h} \in Q_{h}}\left\|\mathbf{y}-\mathbf{x}_{h}\right\|_{0, \Omega} \\
& \left.+h\left\|\mathbf{u}-\mathbf{u}_{d}\right\|_{0, \Omega}+\left\|\boldsymbol{\phi}-\Pi_{h}(\boldsymbol{\phi})\right\|_{0, \Omega}+\left\|\mathbf{y}-\Pi_{h} \mathbf{y}\right\|_{0, \Omega}\right) .
\end{aligned}
$$

Using (4.6), (4.7) and (4.8) in (4.28), we have the result (4.25).

Theorem 4.13. Let $s \in(0,1]$ be the elliptic regularity index. Then there holds

$$
\begin{gathered}
\left\|p-p_{h}\right\|_{0, \Omega} \leq C\left(h^{s}\|\mathbf{u}\|_{1+s, \Omega}+h^{s}\|\boldsymbol{\phi}\|_{1+s, \Omega}+h^{s}\|p\|_{s, \Omega}+h^{s}\|r\|_{s, \Omega}+h^{2}\|\mathbf{y}\|_{1, \Omega}\right. \\
\left.+h\|\mathbf{f}\|_{0, \Omega}+h\left\|\mathbf{u}-\mathbf{u}_{d}\right\|_{0, \Omega}+h\|\boldsymbol{\phi}\|_{1+s, \Omega}+h\|\mathbf{y}\|_{1, \Omega}\right) .
\end{gathered}
$$

Proof. The best approximation result from [4, Theorem 4.1] gives

$$
\begin{aligned}
\left\|p-R_{h} p\right\|_{0, \Omega} \leq C\left(\inf _{\mathbf{v}_{h} \in Z_{h}}\left\|\mathbf{u}-\mathbf{v}_{h}\right\|_{h}+\inf _{s_{h} \in M_{h}}\left\|p-s_{h}\right\|_{0, \Omega}+\right. & \left\|p-P^{0} p\right\|_{0, \Omega} \\
& \left.+h \inf _{\mathbf{x}_{h} \in Q_{h}}\left\|\mathbf{y}-\mathbf{x}_{h}\right\|_{0, \Omega}+h\|\mathbf{f}\|_{0, \Omega}\right) .
\end{aligned}
$$

Using this in the pressure estimate from Theorem 2.5 and (4.26)-(4.27) we have

$$
\left\|p-p_{h}\right\|_{0, \Omega} \leq C\left(\inf _{\mathbf{v}_{h} \in Z_{h}}\left\|\mathbf{u}-\mathbf{v}_{h}\right\|_{h}+\inf _{\mathbf{w}_{h} \in Z_{h}}\left\|\boldsymbol{\phi}-\mathbf{w}_{h}\right\|_{h}+\inf _{s_{h} \in M_{h}}\left\|p-s_{h}\right\|_{0, \Omega}\right.
$$




$$
\begin{aligned}
& +\inf _{s_{h} \in M_{h}}\left\|r-s_{h}\right\|_{0, \Omega}+h\|\mathbf{f}\|_{0, \Omega}+h \inf _{\mathbf{x}_{h} \in Q_{h}}\left\|\mathbf{y}-\mathbf{x}_{h}\right\|_{0, \Omega}+h\left\|\mathbf{u}-\mathbf{u}_{d}\right\|_{0, \Omega} \\
& \left.+\left\|p-P^{0} p\right\|_{0, \Omega}+\left\|\boldsymbol{\phi}-\Pi_{h}(\boldsymbol{\phi})\right\|_{0, \Omega}+\left\|\mathbf{y}-\Pi_{h} \mathbf{y}\right\|_{0, \Omega}\right) .
\end{aligned}
$$

Use of the approximations (4.6), (4.7) and (4.8) in the above equation concludes the proof.

Theorem 4.14. (A posteriori error estimator) There holds,

$$
\begin{gathered}
\left\|\mathbf{y}-\mathbf{y}_{h}\right\|_{0, \Omega}+\left\|\mathbf{u}-\mathbf{u}_{h}\right\|_{h}+\left\|p-p_{h}\right\|_{0, \Omega}+\left\|\boldsymbol{\phi}-\boldsymbol{\phi}_{h}\right\|_{h}+\left\|r-r_{h}\right\|_{0, \Omega} \leq C\left(\eta_{(\mathbf{u}, p)}+\eta_{(\boldsymbol{\phi}, r)}\right. \\
\left.+\left\|\boldsymbol{\phi}_{h}-\Pi_{h} \boldsymbol{\phi}_{h}\right\|_{0, \Omega}\right),
\end{gathered}
$$

where the estimators are defined by,

$$
\begin{aligned}
\eta_{(\mathbf{u}, p)}^{2}= & \sum_{T \in \mathcal{T}_{h}}\left(h_{T}^{2}\left\|\mathbf{f}+\mathbf{y}_{h}\right\|_{0, T}^{2}+\left\|\nabla \cdot \mathbf{u}_{h}\right\|_{0, T}^{2}\right)+\sum_{e \in \mathcal{E}_{h}^{i n t}}\left\|h^{\frac{1}{2}} \llbracket p_{h} I-\nabla \mathbf{u}_{h} \rrbracket\right\|_{0, e}^{2} \\
& +\eta^{2} \sum_{e \in \mathcal{E}_{h}}\left\|h^{-\frac{1}{2}} \underline{\llbracket \mathbf{u}_{h} \rrbracket}\right\|_{0, e}^{2},
\end{aligned}
$$

and

$$
\begin{aligned}
\eta_{(\boldsymbol{\phi}, r)}^{2}= & \sum_{T \in \mathcal{T}_{h}}\left(h_{T}^{2}\left\|\mathbf{u}_{h}-\mathbf{u}_{d}\right\|_{0, T}^{2}+\left\|\nabla \cdot \boldsymbol{\phi}_{h}\right\|_{0, T}^{2}\right)+\sum_{e \in \mathcal{E}_{h}^{i}} \| h^{\frac{1}{2}} \llbracket\left[r_{h} I+\nabla \boldsymbol{\phi}_{h} \rrbracket \|_{0, e}^{2}\right. \\
& +\sigma^{2} \sum_{e \in \mathcal{E}_{h}}\left\|h^{-\frac{1}{2}} \underline{\llbracket \boldsymbol{\phi}_{h} \rrbracket}\right\|_{0, e}^{2} .
\end{aligned}
$$

Proof. Theorem 2.8, 2.9, and 2.10 imply

$$
\begin{array}{r}
\left\|\mathbf{y}-\mathbf{y}_{h}\right\|_{0, \Omega}+\left\|\mathbf{u}-\mathbf{u}_{h}\right\|_{h}+\left\|p-p_{h}\right\|_{0, \Omega}+\left\|\boldsymbol{\phi}-\boldsymbol{\phi}_{h}\right\|_{h}+\left\|r-r_{h}\right\|_{0, \Omega} \leq C\left(\left\|R \mathbf{u}-\mathbf{u}_{h}\right\|_{h}\right. \\
\left.+\left\|R_{0} p-p_{h}\right\|_{0, \Omega}+\left\|\phi_{h}-\bar{R} \boldsymbol{\phi}\right\|_{h}+\left\|\bar{R}_{0} r-r_{h}\right\|_{0, \Omega}+\left\|\phi_{h}-\Pi_{h} \phi_{h}\right\|_{0, \Omega}\right) .
\end{array}
$$

Again, the error analysis in [9, Section 5], [26, Theorem 3.1] allows to conclude the following error estimates:

$$
\begin{aligned}
& \left\|R \mathbf{u}-\mathbf{u}_{h}\right\|_{h}+\left\|R_{0} p-p_{h}\right\|_{0, \Omega} \leq C \eta_{(\mathbf{u}, p)}, \\
& \left\|\bar{R} \boldsymbol{\phi}-\boldsymbol{\phi}_{h}\right\|_{h}+\left\|\bar{R}_{0} r-r_{h}\right\|_{0, \Omega} \leq C \eta_{(\boldsymbol{\phi}, r)} .
\end{aligned}
$$

Now using (4.30) and (4.31) in (4.29), we complete the proof of the theorem.

The standard bubble function techniques can deduce the following theorem on local efficiency:

Theorem 4.15. (Efficiency) Let $\mathcal{T}_{e}$ be the set of two triangles sharing the edge $e \in \mathcal{E}_{h}^{i}$. Then there holds

$$
\begin{aligned}
h_{T}\left\|\mathbf{f}+\mathbf{y}_{h}\right\|_{0, T} & \leq C\left(\left\|\nabla\left(\mathbf{u}-\mathbf{u}_{h}\right)\right\|_{0, T}+\left\|p-p_{h}\right\|_{0, T}+\operatorname{osc}(\mathbf{f}, \mathbf{T})+\left\|\mathbf{y}-\mathbf{y}_{h}\right\|_{0, T}\right), \\
h_{T}\left\|\mathbf{u}_{h}-\mathbf{u}_{d}\right\|_{0, T} & \leq C\left(\left\|\mathbf{u}-\mathbf{u}_{h}\right\|_{0, T}+\left\|\nabla\left(\boldsymbol{\phi}-\boldsymbol{\phi}_{h}\right)\right\|_{0, T}+\left\|r-r_{h}\right\|_{0, T}+\operatorname{osc}\left(\mathbf{u}_{d}, T\right)\right),
\end{aligned}
$$




$$
\begin{aligned}
\left.\| h^{\frac{1}{2}} \llbracket p_{h} I-\nabla \mathbf{u}_{h}\right] \|_{0, e} & \leq C \sum_{T \in \mathcal{T}_{e}}\left(\left\|\nabla\left(\mathbf{u}-\mathbf{u}_{h}\right)\right\|_{0, T}+\left\|p-p_{h}\right\|_{0, T}+\operatorname{osc}(\mathbf{f}, T)+\left\|\mathbf{y}-\mathbf{y}_{h}\right\|_{0, T}\right) \\
\left\|h^{\frac{1}{2}}\left[r_{h} I+\nabla \boldsymbol{\phi}_{h}\right] \rrbracket\right\|_{0, e} & \leq C \sum_{T \in \mathcal{T}_{e}}\left(\left\|\mathbf{u}-\mathbf{u}_{h}\right\|_{0, T}+\left\|\nabla\left(\boldsymbol{\phi}-\boldsymbol{\phi}_{h}\right)\right\|_{0, T}+\left\|r-r_{h}\right\|_{0, T}+\operatorname{osc}\left(\mathbf{u}_{d}, T\right)\right) \\
\left\|\nabla \cdot \mathbf{u}_{h}\right\|_{0, T} & \leq C\left\|\nabla\left(\mathbf{u}-\mathbf{u}_{h}\right)\right\|_{0, T} \\
\left\|\nabla \cdot \boldsymbol{\phi}_{h}\right\|_{0, T} & \leq C\left\|\nabla\left(\boldsymbol{\phi}-\boldsymbol{\phi}_{h}\right)\right\|_{0, T} \\
\left\|\boldsymbol{\phi}_{h}-\Pi_{h} \boldsymbol{\phi}_{h}\right\|_{0, T} & \leq C\left(\left\|\boldsymbol{\phi}_{h}-\boldsymbol{\phi}\right\|_{0, T}+\left\|\boldsymbol{\phi}-\Pi_{h} \boldsymbol{\phi}_{h}\right\|_{0, T}\right)
\end{aligned}
$$

4.3.2. Discrete boundary control problem: The model problem in this section is the model problem 2 introduced in the section 3. Set

$$
X=\left[H^{1}(\Omega) \cap L_{0}^{2}(\Omega)\right]^{2}, W=\left[L^{2}(\Omega)\right]^{2}, M=L^{2}(\Omega) \text { and } Q=\left[L^{2}(\Gamma)\right]^{2} .
$$

The set $Q_{a d}=Q_{b}$, where $Q_{b}$ is defined in section 3. The discrete spaces

$$
X_{h}:=\left[L_{0}^{2}(\Omega)\right]^{2} \cap\left[\mathbb{P}_{1}(\mathcal{T})\right]^{2} \text {, and } M_{h}:=\mathbb{P}_{0}(\mathcal{T})
$$

Define discrete control space $Q_{h}=\left\{\mathbf{x}_{h} \in\left[L^{2}(\Gamma)\right]^{2}:\left.\mathbf{x}_{h}\right|_{e} \in\left[\mathbb{P}_{0}(e)\right]^{2}\right.$ for all $\left.e \in \mathcal{E}_{h}^{b}\right\}$, and the admissible control set $Q_{a d}^{h}=\left\{\mathbf{x}_{h} \in Q_{h}: \int_{\Gamma} \mathbf{x}_{\mathbf{h}} \mathrm{ds}+\int_{\Omega} \mathbf{f} \mathrm{dx}=\mathbf{0}, \quad \mathbf{y}_{a} \leq \mathbf{x}_{h} \leq \mathbf{y}_{b}\right\}$.

It is clear that $Q_{a d}^{h} \subseteq Q_{a d}$ and $\Pi_{h} \mathbf{u} \in Q_{a d}^{h}$ for $\mathbf{u} \in Q_{a d}$. The operator $E: X \rightarrow Q$ is the trace map and $E_{h}: X_{h} \rightarrow Q_{h}$ is defined by the piecewise (edge-wise) trace, i.e., $\left.E_{h} \mathbf{w}\right|_{e}=\left.\mathbf{w}_{T}\right|_{e}$ where $\mathbf{w}_{T}=\left.\mathbf{w}\right|_{T}$ and $T$ be the triangle having the edge $e$ on boundary. The DG bilinear form for the diffusion term is given by

$$
\begin{aligned}
a_{h}\left(\mathbf{v}_{h}, \mathbf{z}_{h}\right)= & \sum_{T \in \mathcal{T}_{h}} \int_{T} \nabla \mathbf{v}_{h}: \nabla \mathbf{z}_{h} \mathrm{dx}-\sum_{e \in \mathcal{E}_{h}^{i}} \int_{e}\left\{\left\{\nabla \mathbf{v}_{h}\right\}\right\}: \underline{\llbracket \mathbf{z}_{h} \rrbracket} \mathrm{ds}-\sum_{e \in \mathcal{E}_{h}^{i}} \int_{e}\left\{\left\{\nabla \mathbf{z}_{h}\right\}\right\}: \underline{\llbracket \mathbf{v}_{h} \rrbracket} \mathrm{ds} \\
& +\sum_{e \in \mathcal{E}_{h}^{i}} \frac{\sigma}{h_{e}} \int_{e} \underline{\llbracket \mathbf{v}_{h} \rrbracket}: \underline{\llbracket \mathbf{z}_{h} \rrbracket} \mathrm{ds},
\end{aligned}
$$

for all $\mathbf{v}_{h}, \mathbf{z}_{h} \in X_{h}$ and $\sigma>0$ be a real number. The DG bilinear form for the pressure term is given by

$$
b_{h}\left(\mathbf{z}_{h}, p_{h}\right)=-\sum_{T \in \mathcal{T}_{h}} \int_{T} p_{h} \nabla \cdot \mathbf{z}_{h} \mathrm{dx}+\sum_{e \in \mathcal{E}_{h}^{i}} \int_{e}\left\{\left\{p_{h}\right\}\right\} \llbracket\left[\mathbf{z}_{h} \rrbracket \mathrm{ds},\right.
$$

for all $\mathbf{z}_{h} \in X_{h}$ and $p_{h} \in M_{h}$. After integration by parts on the right-hand side of (4.33) we have

$$
b_{h}\left(\mathbf{z}_{h}, p_{h}\right)=\sum_{T \in \mathcal{T}_{h}} \int_{T} \mathbf{z}_{h} \cdot \nabla p_{h} \mathrm{dx}-\sum_{e \in \mathcal{E}_{h}} \int_{e} \llbracket p_{h} \rrbracket\left\{\left\{\mathbf{z}_{h}\right\}\right\} \mathrm{ds},
$$

for all $\mathbf{z}_{h} \in X_{h}$ and $p_{h} \in M_{h}$. We choose $\sigma>0$ large enough such that $a_{h}(\cdot, \cdot)$ is $X_{h}$-elliptic with respect to the norm $\|\cdot\|_{h}$ on $X_{h}$ which is given by $\left\|\mathbf{v}_{h}\right\|_{h}^{2}=\sum_{T \in \mathcal{T}_{h}} \int_{T}\left|\nabla \mathbf{v}_{h}\right|^{2} \mathrm{dx}+$ $\sum_{e \in \mathcal{E}_{h}^{i}} \frac{1}{h_{e}} \int_{e}{\underline{\llbracket \mathbf{v}_{h} \rrbracket^{2}}}^{\mathrm{d}} \mathrm{ds}$ and $b_{h}$ satisfies the inf-sup condition. The inequality (2.4) follows from 
the results Poincaré-Friedrichs type inequalities in [6]. The estimate in (2.5) follows from the well-known trace inequality and [6].

Theorem 4.16. Let $s \in(0,1]$ be the elliptic regularity index. Then there holds

$$
\begin{aligned}
& \left\|\mathbf{y}-\mathbf{y}_{h}\right\|_{0, \Gamma}+\left\|\boldsymbol{\phi}-\boldsymbol{\phi}_{h}\right\|_{h}+\left\|\mathbf{u}-\mathbf{u}_{h}\right\|_{h} \leq C\left(h^{s}\|\mathbf{u}\|_{1+s, \Omega}+h^{s}\|\boldsymbol{\phi}\|_{1+s, \Omega}+h^{s}\|p\|_{s, \Omega}+h^{s}\|r\|_{s, \Omega}\right. \\
& \left.(4.35)+\sum_{i=1}^{m} h^{1+s}\|\mathbf{y}\|_{\frac{1}{2}+s, \Gamma_{i}}+h\|\mathbf{f}\|_{0, \Omega}+h\left\|\mathbf{u}-\mathbf{u}_{d}\right\|_{0, \Omega}+h\|\boldsymbol{\phi}\|_{1+s, \Omega}+\sum_{i=1}^{m} h^{\frac{1}{2}+s}\|\mathbf{y}\|_{\frac{1}{2}+s, \Gamma_{i}}\right) .
\end{aligned}
$$

Proof. From Theorem 2.4 we have

$$
\left\|\boldsymbol{\phi}-\boldsymbol{\phi}_{h}\right\|_{h} \leq C\left(\left\|\boldsymbol{\phi}-\bar{P}_{h} \boldsymbol{\phi}\right\|_{h}+\left\|\boldsymbol{\phi}-\Pi_{h}(\boldsymbol{\phi})\right\|_{0, \Gamma}+\left\|\mathbf{y}-\Pi_{h} \mathbf{y}\right\|_{0, \Gamma}+\left\|\mathbf{u}-P_{h} \mathbf{u}\right\|_{h}\right),
$$

and

$$
\left\|\mathbf{u}-\mathbf{u}_{h}\right\|_{h} \leq C\left(\left\|\mathbf{u}-P_{h} \mathbf{u}\right\|_{h}+\left\|\boldsymbol{\phi}-\Pi_{h}(\boldsymbol{\phi})\right\|_{0, \Gamma}+\left\|\mathbf{y}-\Pi_{h} \mathbf{y}\right\|_{0, \Gamma}+\left\|\boldsymbol{\phi}-\bar{P}_{h} \boldsymbol{\phi}\right\|_{h}\right) .
$$

From [4, Theorem 3.1], we have

$$
\left\|\boldsymbol{\phi}-\bar{P}_{h} \boldsymbol{\phi}\right\|_{h} \leq C\left(\inf _{\mathbf{w}_{h} \in Z_{h}}\left\|\boldsymbol{\phi}-\mathbf{w}_{h}\right\|_{h}+\inf _{s_{h} \in M_{h}}\left\|r-s_{h}\right\|_{0, \Omega}+h\left\|\mathbf{u}-\mathbf{u}_{d}\right\|_{0, \Omega}\right)
$$

and

$$
\left\|\mathbf{u}-\bar{P}_{h} \mathbf{u}\right\|_{h} \leq C\left(\inf _{\mathbf{v}_{h} \in Z_{h}}\left\|\mathbf{u}-\mathbf{v}_{h}\right\|_{h}+\inf _{s_{h} \in M_{h}}\left\|p-s_{h}\right\|_{0, \Omega}+h\|\mathbf{f}\|_{0, \Omega}+h^{\frac{1}{2}} \inf _{\mathbf{x}_{h} \in Q_{h}}\left\|\mathbf{y}-\mathbf{x}_{h}\right\|_{0, \Gamma}\right) .
$$

From Theorem 2.3, we have

$$
\left\|\mathbf{y}-\mathbf{y}_{h}\right\|_{0, \Gamma} \leq C\left(\left\|\boldsymbol{\phi}-\Pi_{h} \boldsymbol{\phi}\right\|_{0, \Gamma}+\left\|\mathbf{y}-\Pi_{h} \mathbf{y}\right\|_{0, \Gamma}+\left\|\boldsymbol{\phi}-\bar{P}_{h} \phi\right\|_{h}+\left\|\mathbf{u}-P_{h} \mathbf{u}\right\|_{h}\right) .
$$

The above estimates yield

$$
\begin{aligned}
\left\|\mathbf{y}-\mathbf{y}_{h}\right\|_{0, \Gamma}+\left\|\boldsymbol{\phi}-\boldsymbol{\phi}_{h}\right\|_{h}+ & \left\|\mathbf{u}-\mathbf{u}_{h}\right\|_{h} \leq C\left(\inf _{\mathbf{v}_{h} \in Z_{h}}\left\|\mathbf{u}-\mathbf{v}_{h}\right\|_{h}+\inf _{\mathbf{w}_{h} \in Z_{h}}\left\|\boldsymbol{\phi}-\mathbf{w}_{h}\right\|_{h}\right. \\
& +\inf _{s_{h} \in M_{h}}\left\|p-s_{h}\right\|_{0, \Omega}+\inf _{s_{h} \in M_{h}}\left\|r-s_{h}\right\|_{0, \Omega}+h^{\frac{1}{2}} \inf _{\mathbf{x}_{h} \in Q_{h}}\left\|\mathbf{y}-\mathbf{x}_{h}\right\|_{0, \Gamma} \\
& \left.+h\|\mathbf{f}\|_{0, \Omega}+h\left\|\mathbf{u}-\mathbf{u}_{d}\right\|_{0, \Omega}+\left\|\boldsymbol{\phi}-\Pi_{h} \boldsymbol{\phi}\right\|_{0, \Gamma}+\left\|\mathbf{y}-\Pi_{h} \mathbf{y}\right\|_{0, \Gamma}\right) .
\end{aligned}
$$

Now we can apply elliptic regularity to derive concrete error estimates. Note that by wellposedness of the problem, $\mathbf{u}, \boldsymbol{\phi} \in\left[H^{1}(\Omega) \cap L_{0}^{2}(\Omega)\right]^{2}$ and $p \in L^{2}(\Omega)$. The elliptic regularity of polygonal domains implies that $\phi \in\left[H^{1+s}(\Omega)\right]^{2} \cap X$ and $r \in H^{s}(\Omega)$ for some $s \in(0,1]$, which depends on the interior angles of the domain $\Omega$. We know that, $\mathbf{y}=\Pi_{\left[\mathbf{y}_{a}, \mathbf{y}_{b}\right]}\left(-\frac{\phi}{\lambda}\right)$ on $\partial \Omega$. Hence the control $\left.\mathbf{y}\right|_{\Gamma_{i}} \in\left[H^{\frac{1}{2}+s}\left(\Gamma_{i}\right)\right]^{2}$ for $1 \leq i \leq m$ and $\mathbf{u} \in\left[H^{1+s}(\Omega)\right]^{2} \cap X$ and $p \in H^{s}(\Omega)$, where $m$ is the number of boundary edges. Also we have the following estimates,

$$
\left\|\boldsymbol{\phi}-\Pi_{h} \phi\right\|_{0, \Gamma} \leq C h^{\frac{1}{2}+s}\|\boldsymbol{\phi}\|_{1+s, \Omega}, \text { and }\left\|\mathbf{y}-\Pi_{h} \mathbf{y}\right\|_{0, \Gamma} \leq C \sum_{i=1}^{m} h^{\frac{1}{2}+s}\|\mathbf{y}\|_{\frac{1}{2}+s, \Gamma_{i}} .
$$

Finally, substitution of the estimates from (4.6), (4.7) and (4.39) in (4.38) leads to (4.35), and this concludes the proof. 
Theorem 4.17. Let $s \in(0,1]$ be the elliptic regularity index. Then there holds

$$
\begin{gathered}
\left\|p-p_{h}\right\|_{0, \Omega} \leq C \\
+h\|\mathbf{h}\| \mathbf{u}\left\|_{1+s, \Omega}+h^{s}\right\| \boldsymbol{\phi}\left\|_{1+s, \Omega}+h\right\| \mathbf{u}-h^{s}\|p\|_{s, \Omega}+h^{s}\|r\|_{s, \Omega}+\sum_{i=1}^{m} h^{1+s}\|\mathbf{y}\|_{\frac{1}{2}+s, \Gamma_{i}} \\
\left.+h\|\boldsymbol{\phi}\|_{1+s, \Omega}\right) .
\end{gathered}
$$

Proof. From Theorem 2.5, we have pressure estimate

$$
\begin{aligned}
\left\|p-p_{h}\right\|_{0, \Omega} \leq & C\left(\left\|p-R_{h} p\right\|_{0, \Omega}+\left\|\mathbf{u}-P_{h} \mathbf{u}\right\|_{h}+\left\|\boldsymbol{\phi}-\bar{P}_{h} \boldsymbol{\phi}\right\|_{h}\right. \\
& \left.+\left\|\boldsymbol{\phi}-\Pi_{h}(\boldsymbol{\phi})\right\|_{0, \Gamma}+\left\|\mathbf{y}-\Pi_{h} \mathbf{y}\right\|_{0, \Gamma}\right) .
\end{aligned}
$$

As a consequence of the error analysis in [4, Theorem 4.1] for Neumann boundary value problem, we have

$$
\begin{aligned}
\left\|p-R_{h} p\right\|_{0, \Omega} \leq & C\left(\inf _{\mathbf{v}_{h} \in Z_{h}}\left\|\mathbf{u}-\mathbf{v}_{h}\right\|_{h}+\left\|p-P^{0} p\right\|_{0, \Omega}+\inf _{q_{h} \in M_{h}}\left\|p-q_{h}\right\|_{0, \Omega}\right. \\
& \left.+h^{\frac{1}{2}} \inf _{\mathbf{x}_{h} \in Q_{h}}\left\|\mathbf{y}-\mathbf{x}_{h}\right\|_{0, \Gamma}+h\|\mathbf{f}\|_{0, \Omega}\right) .
\end{aligned}
$$

Using the (4.36), (4.37) and the above estimate we get

$$
\begin{aligned}
\left\|p-p_{h}\right\|_{0, \Omega} \leq C & \left(\inf _{\mathbf{v}_{h} \in Z_{h}}\left\|\mathbf{u}-\mathbf{v}_{h}\right\|_{h}+\inf _{\mathbf{w}_{h} \in Z_{h}}\left\|\boldsymbol{\phi}-\mathbf{w}_{h}\right\|_{h}+\inf _{s_{h} \in M_{h}}\left\|p-s_{h}\right\|_{0, \Omega}\right. \\
& +\inf _{s_{h} \in M_{h}}\left\|r-s_{h}\right\|_{0, \Omega}+h\|\mathbf{f}\|_{0, \Omega}+h^{\frac{1}{2}} \inf _{\mathbf{x}_{h} \in Q_{h}}\left\|\mathbf{y}-\mathbf{x}_{h}\right\|_{0, \Gamma}+h\left\|\mathbf{u}-\mathbf{u}_{d}\right\|_{0, \Omega} \\
& \left.+\left\|p-P^{0} p\right\|_{0, \Omega}+\left\|\boldsymbol{\phi}-\Pi_{h} \boldsymbol{\phi}\right\|_{0, \Omega}+\left\|\mathbf{y}-\Pi_{h} \mathbf{y}\right\|_{0, \Gamma}\right) .
\end{aligned}
$$

By using (4.6), (4.7) and (4.39) we have the pressure estimate.

Theorem 4.18. (A posteriori error estimator) There holds,

$$
\left\|\mathbf{y}-\mathbf{y}_{h}\right\|_{0, \Gamma}+\left\|\mathbf{u}-\mathbf{u}_{h}\right\|_{h}+\left\|p-p_{h}\right\|_{0, \Omega}+\left\|\boldsymbol{\phi}-\boldsymbol{\phi}_{h}\right\|_{h}+\left\|r-r_{h}\right\|_{0, \Omega} \leq C\left(\eta_{(\mathbf{u}, p)}+\eta_{(\phi, r)}\right.
$$

where the estimators are defined by,

$$
\left.+\left\|\phi_{h}-\Pi_{h} \phi_{h}\right\|_{0, \Gamma}\right) \text {. }
$$

$$
\begin{aligned}
\eta_{(\mathbf{u}, p)}^{2}= & \sum_{T \in \mathcal{T}_{h}}\left(h_{T}^{2}\|\mathbf{f}\|_{0, T}^{2}+\left\|\nabla \cdot \mathbf{u}_{h}\right\|_{0, T}^{2}\right)+\sum_{e \in \mathcal{E}_{h}^{i}}\left\|h^{\frac{1}{2}} \llbracket p_{h} I-\nabla \mathbf{u}_{h} \rrbracket\right\|_{0, e}^{2} \\
& +\sum_{e \in \mathcal{E}_{h}^{b}}\left\|h^{\frac{1}{2}}\left(p_{h} I-\nabla \mathbf{u}_{h}+\mathbf{y}_{h} I\right)\right\|_{0, e}^{2}+\sigma^{2} \sum_{e \in \mathcal{E}_{h}^{i}}\left\|h^{-\frac{1}{2}} \underline{\llbracket \mathbf{u}_{h} \rrbracket}\right\|_{0, e}^{2},
\end{aligned}
$$

and

$$
\begin{aligned}
\eta_{(\boldsymbol{\phi}, r)}^{2}= & \sum_{T \in \mathcal{T}_{h}}\left(h_{T}^{2}\left\|\mathbf{u}_{h}-\mathbf{u}_{d}\right\|_{0, T}^{2}+\left\|\nabla \cdot \boldsymbol{\phi}_{h}\right\|_{0, T}^{2}\right)+\sum_{e \in \mathcal{E}_{h}^{i}}\left\|h^{\frac{1}{2}} \llbracket r_{h} I+\nabla \boldsymbol{\phi}_{h} \rrbracket\right\|_{0, e}^{2} \\
& +\sum_{e \in \mathcal{E}_{h}^{b}}\left\|h^{\frac{1}{2}}\left(r_{h} I+\nabla \boldsymbol{\phi}_{h}\right)\right\|_{0, e}^{2}+\sigma^{2} \sum_{e \in \mathcal{E}_{h}^{i}}\left\|h^{-\frac{1}{2}} \underline{\llbracket \boldsymbol{\phi}_{h} \rrbracket}\right\|_{0, e}^{2} \cdot
\end{aligned}
$$


Proof. From Theorem 2.8, 2.9] and 2.10, we get

$$
\begin{array}{r}
\left\|\mathbf{y}-\mathbf{y}_{h}\right\|_{0, \Gamma}+\left\|\mathbf{u}-\mathbf{u}_{h}\right\|_{h}+\left\|p-p_{h}\right\|_{0, \Omega}+\left\|\boldsymbol{\phi}-\phi_{h}\right\|_{h}+\left\|r-r_{h}\right\|_{0, \Omega} \leq C\left(\left\|R \mathbf{u}-\mathbf{u}_{h}\right\|_{h}\right. \\
\left.+\left\|R_{0} p-p_{h}\right\|_{0, \Omega}+\left\|\phi_{h}-\bar{R} \phi\right\|_{h}+\left\|R_{0} r-r_{h}\right\|_{0, \Omega}+\left\|\phi_{h}-\Pi_{h} \phi_{h}\right\|_{0, \Gamma}\right) .
\end{array}
$$

As a consequence of the error analysis in [9, Section 5], [26, Theorem 3.1] for Neumann boundary value problem conclude the following error estimates:

$$
\begin{aligned}
& \left\|R \mathbf{u}-\mathbf{u}_{h}\right\|_{h}+\left\|R_{0} p-p_{h}\right\|_{0, \Omega} \leq C \eta_{(\mathbf{u}, p)}, \\
& \left\|\bar{R} \boldsymbol{\phi}-\boldsymbol{\phi}_{h}\right\|_{h}+\left\|\bar{R}_{0} r-r_{h}\right\|_{0, \Omega} \leq C \eta_{(\boldsymbol{\phi}, r)} .
\end{aligned}
$$

Now, the substitution of (4.42) and (4.43) in (4.41) concludes the proof of (4.40).

Theorem 4.19. (Efficiency) Let $\mathcal{T}_{e}$ be the set of two triangles sharing the edge $e \in \mathcal{E}_{h}^{i}$. Then there hold

$$
\begin{aligned}
h_{T}\|\mathbf{f}\|_{0, T} & \leq C\left(\left\|\nabla\left(\mathbf{u}-\mathbf{u}_{h}\right)\right\|_{0, T}+\left\|p-p_{h}\right\|_{0, T}+\operatorname{osc}(\mathbf{f}, T)\right), \\
h_{T}\left\|\mathbf{u}_{h}-\mathbf{u}_{d}\right\|_{0, T} & \leq C\left(\left\|\mathbf{u}-\mathbf{u}_{h}\right\|_{0, T}+\left\|\nabla\left(\boldsymbol{\phi}-\boldsymbol{\phi}_{h}\right)\right\|_{0, T}+\left\|r-r_{h}\right\|_{0, T}+\operatorname{osc}\left(\mathbf{u}_{d}, T\right)\right), \\
\left\|h^{\frac{1}{2}} \llbracket p_{h} I-\nabla \mathbf{u}_{h} \rrbracket\right\|_{0, e} & \leq C \sum_{T \in \mathcal{T}_{e}}\left(\left\|\nabla\left(\mathbf{u}-\mathbf{u}_{h}\right)\right\|_{0, T}+\left\|p-p_{h}\right\|_{0, T}+\operatorname{osc}(\mathbf{f}, T)+\left\|\mathbf{y}-\mathbf{y}_{h}\right\|_{0, T}\right), \\
\left\|\nabla \cdot \mathbf{u}_{h}\right\|_{0, T} & \leq C\left\|\nabla\left(\mathbf{u}-\mathbf{u}_{h}\right)\right\|_{0, T}, \\
\left\|\nabla \cdot \boldsymbol{\phi}_{h}\right\|_{0, T} & \leq C\left\|\nabla\left(\boldsymbol{\phi}-\boldsymbol{\phi}_{h}\right)\right\|_{0, T}, \\
\left\|\boldsymbol{\phi}_{h}-\Pi_{h} \boldsymbol{\phi}_{h}\right\|_{0, T} & \leq C\left(\left\|\boldsymbol{\phi}_{h}-\boldsymbol{\phi}\right\|_{0, T}+\left\|\boldsymbol{\phi}-\Pi_{h} \boldsymbol{\phi}_{h}\right\|_{0, T}\right) .
\end{aligned}
$$

Further for any boundary edge $e \in \mathcal{E}_{h}^{b}$, there hold

$$
\begin{aligned}
\left\|h^{\frac{1}{2}}\left(p_{h} I-\nabla \mathbf{u}_{h}+\mathbf{y}_{h} I\right)\right\|_{0, e} & \leq C\left(\left\|\nabla\left(\mathbf{u}-\mathbf{u}_{h}\right)\right\|_{0, T}+\left\|p-p_{h}\right\|_{0, T}+o s c(\mathbf{f}, T)+\left\|\mathbf{y}-\mathbf{y}_{h}\right\|_{0, T}\right) \\
\left\|h^{\frac{1}{2}}\left(r_{h} I+\nabla \boldsymbol{\phi}_{h}\right)\right\|_{0, e} & \leq C\left(\left\|\mathbf{u}-\mathbf{u}_{h}\right\|_{0, T}+\left\|\nabla\left(\boldsymbol{\phi}-\boldsymbol{\phi}_{h}\right)\right\|_{0, T}+\left\|r-r_{h}\right\|_{0, T}+\operatorname{osc}\left(\mathbf{u}_{d}, T\right)\right)
\end{aligned}
$$

Remark 4.20. The analysis can be extended to the three dimensions also for the simplicity we strict ourselves to two dimensions.

\section{NumERICAL EXPERIMENTS}

This section presents some numerical experiments to illustrate the theoretical results derived in the article. The abstract framework of a priori and a posteriori error analysis is applicable for the set of discrete spaces $X_{h} \times M_{h} \times Q_{a d}^{h}$ for the approximation of velocity, pressure, and control in conforming, nonconforming FEM and discontinuous Galerkin methods as discussed in Section 4. Here in the following the numerical experiments, we have considered $\mathrm{CR}_{0}^{1}\left(\mathcal{T}_{h}\right) \times\left(\mathbb{P}_{0}\left(\mathcal{T}_{h}\right) \cap L_{0}^{2}(\Omega)\right) \times\left(\mathbb{P}_{0}\left(\mathcal{T}_{h}\right) \cap Q_{d}\right)$ spaces for the approximations. 
Example 5.1. Consider the optimal control problem (3.10a)-(3.10c) with the domain $\Omega=$ $(0,1)^{2}$ and the exact solution

$$
\mathbf{u}=\boldsymbol{\phi}=\left(\begin{array}{c}
\sin ^{2}(\pi x) \sin (\pi y) \cos (\pi y) \\
-\sin ^{2}(\pi y) \sin (\pi x) \cos (\pi x)
\end{array}\right), p=r=\sin (2 \pi x) \sin (2 \pi y),
$$

and $\mathbf{y}=\Pi_{[a, b]}\left(-\frac{1}{\lambda} \mathbf{u}\right)$, where $\mathbf{y}_{a}=-0.1, \mathbf{y}_{b}=0.25$. The data of the problem are chosen such that

$$
\mathbf{f}=-\Delta \mathbf{u}+\nabla p-\mathbf{y}, \text { and } \mathbf{u}_{d}=\mathbf{u}+\Delta \boldsymbol{\phi}+\nabla r .
$$

In this numerical simulation, the $\mathrm{CR}_{0}^{1} / \mathbb{P}_{0}$ pair is used for the approximations of state and adjoint state velocity and pressure variables, and piecewise constant space for the control variable. For the computation of the discrete solution, the primal-dual algorithm [34, pp. $100]$ is used. The discrete approximations for the state velocity variables using nonconforming finite elements are shown in Figure 5.1 and the discrete approximations for the control variable $\mathbf{y}$ using piecewise constant elements are shown in Figure 5.2 .
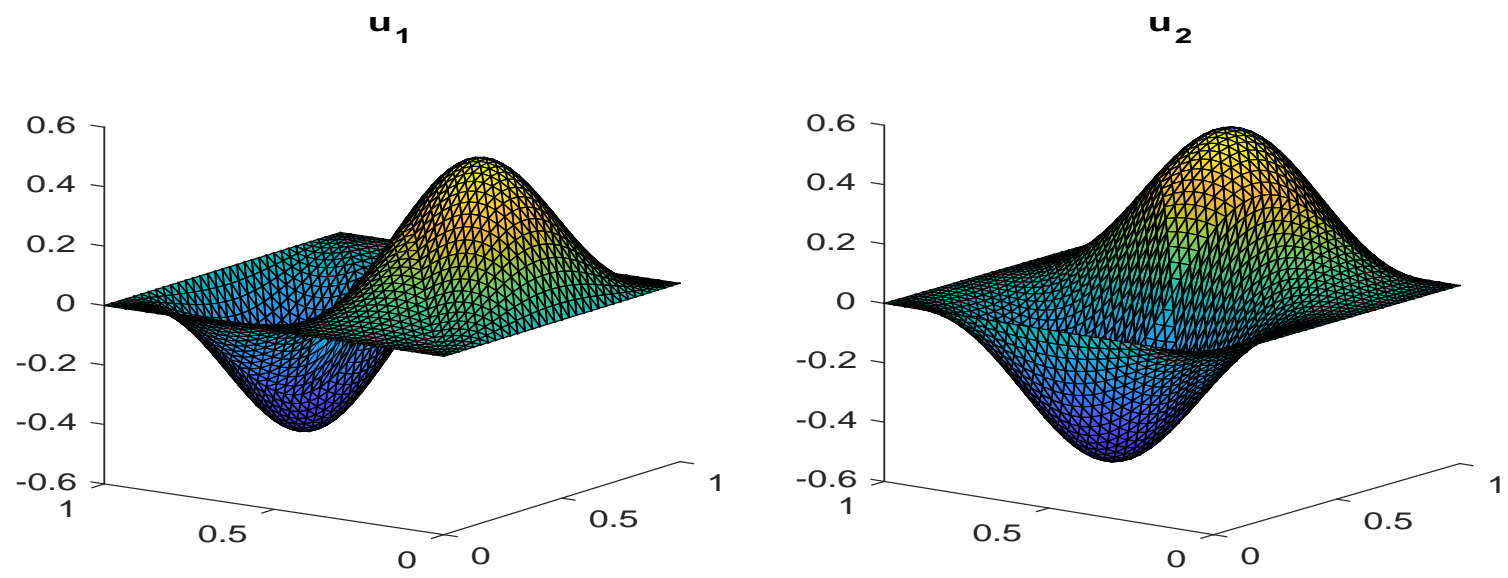

Figure 5.1. Discrete velocity $\mathbf{u}_{h}=\left(u_{1}, u_{2}\right)$ for Example 5.1

Table 5.1 displays the errors and convergence rates of FE approximations. The linear convergence is observed for error in approximation of state and adjoint state velocity in energy norm, and also for state pressure, adjoint pressure and control variables in $L_{2}$-norm. Moreover, we have also observed the quadratic convergence in $L^{2}$-norm for state and adjoint state velocity variables.

Example 5.2. Consider the optimal control problem (3.10a)-(3.10c) with the L-shaped domain $\Omega=(-1,1)^{2} \backslash((0,1) \times(-1,0))$ and the exact solution

$$
\begin{array}{r}
\mathbf{u}=r^{\alpha}\left(\begin{array}{c}
(1+\alpha) \sin (\theta) \omega(\theta)+\cos (\theta) \omega^{\prime}(\theta) \\
-(1+\alpha) \cos (\theta) \omega(\theta)+\sin (\theta) \omega^{\prime}(\theta)
\end{array}\right), \\
p=-r^{\alpha-1}\left((1+\alpha)^{2} \omega^{\prime}(\theta)+\omega^{\prime \prime \prime}(\theta)\right) /(1-\alpha),
\end{array}
$$



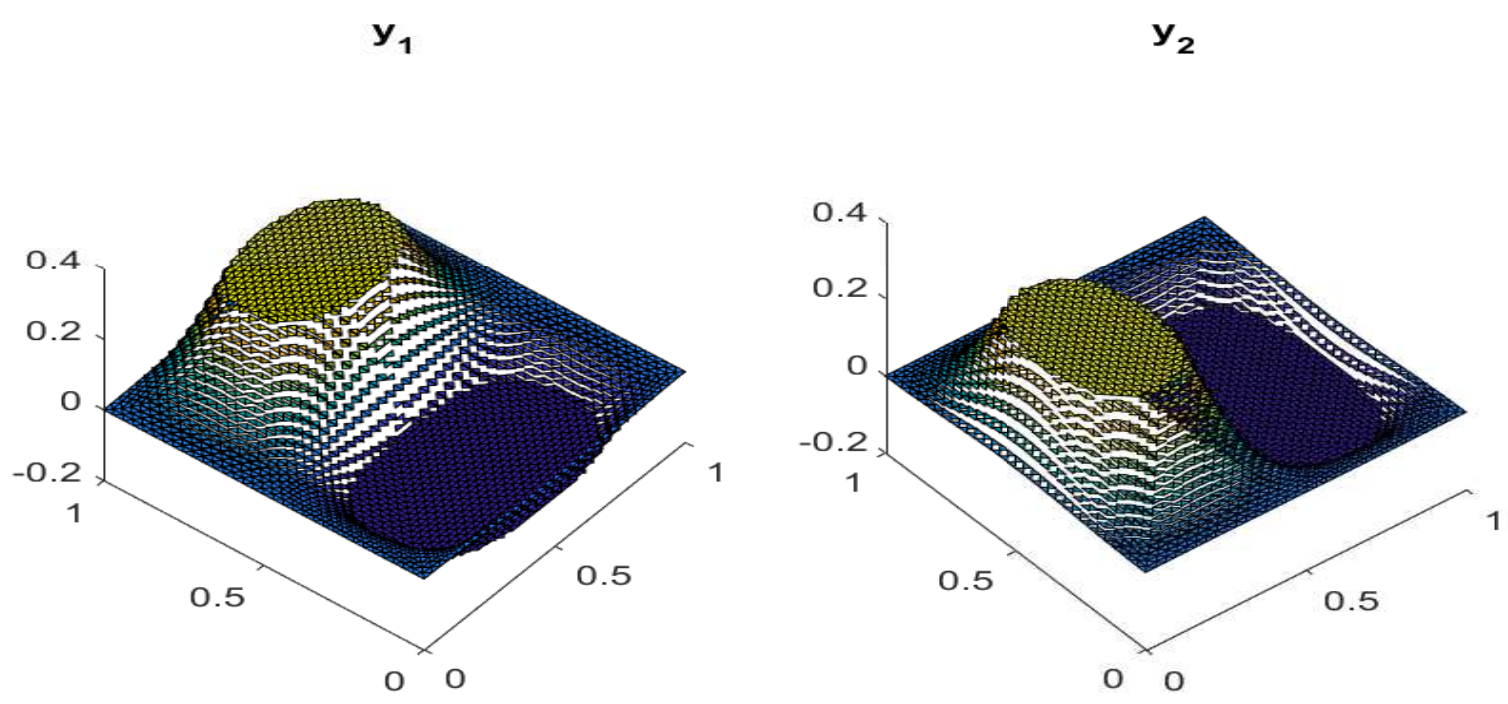

Figure 5.2. Discrete control $\mathbf{y}_{h}=\left(y_{1}, y_{2}\right)$ of Example 5.1

\begin{tabular}{c||c|c|c|c|c|c|c|c|c|c}
$h$ & $\left\|\mathbf{u}-\mathbf{u}_{h}\right\|_{h}$ & $\mathrm{CR}$ & $\left\|p-p_{h}\right\|$ & $\mathrm{CR}$ & $\left\|\boldsymbol{\phi}-\boldsymbol{\phi}_{h}\right\|_{h}$ & $\mathrm{CR}$ & $\left\|r-r_{h}\right\|$ & $\mathrm{CR}$ & $\left\|\mathbf{y}-\mathbf{y}_{h}\right\|$ & $\mathrm{CR}$ \\
\hline \hline 0.2500 & 0.8877 & 0 & 0.9362 & 0 & 0.8888 & 0 & 0.9360 & 0 & 0.0985 & 0 \\
0.1250 & 0.5350 & 0.73 & 0.3511 & 1.41 & 0.5351 & 0.73 & 0.3510 & 1.41 & 0.0556 & 0.82 \\
0.0625 & 0.2680 & 0.99 & 0.1682 & 1.06 & 0.2680 & 0.99 & 0.1682 & 1.06 & 0.0296 & 0.91 \\
0.0312 & 0.1346 & 0.99 & 0.0819 & 1.03 & 0.1346 & 0.99 & 0.0819 & 1.03 & 0.0152 & 0.96 \\
0.0156 & 0.0674 & 0.99 & 0.0406 & 1.01 & 0.0674 & 0.99 & 0.0406 & 1.01 & 0.0076 & 0.98 \\
0.0078 & 0.0337 & 0.99 & 0.0202 & 1.00 & 0.0337 & 0.99 & 0.0202 & 1.00 & 0.0038 & 0.99 \\
\hline
\end{tabular}

TABLE 5.1. Errors and convergence rates (CR) for the Example 5.1.

where

$$
\begin{aligned}
\omega(\theta)= & 1 /(1+\alpha) \sin (\alpha+1) \theta) \cos (\alpha w)-\cos ((\alpha+1) \theta) \\
& +1 /(1+\alpha) \sin (\alpha-1) \theta) \cos (\alpha \omega)-\cos ((\alpha-1) \theta)
\end{aligned}
$$

and $\alpha=856399 / 1572864$ and $w=3 \pi / 2$. The adjoint variables $\phi, r$ are considered as same as in Example 5.1 and $\mathbf{y}=\Pi_{[a, b]}\left(-\frac{1}{\lambda} \phi\right)$, where $\mathbf{y}_{a}=-0.1, \mathbf{y}_{b}=0.25$. The data of the problem is chosen such that

$$
\mathbf{f}=-\Delta \mathbf{u}+\nabla p-\mathbf{y}, \text { and } \mathbf{u}_{d}=\mathbf{u}+\Delta \boldsymbol{\phi}+\nabla r .
$$

This problem is defined on the L-shaped domain, and the solution $(\mathbf{u}, p)$ has a singularity at the origin. It is known that for this problem the uniform refinements will not provide an optimal convergence rate. We have similar observation from Figure 5.5, for uniform refinements convergence rate with respect to the number of degrees of freedom (Ndof) is 0.25 (that is, respect to the mesh-size $h \equiv \operatorname{Ndof}^{-1 / 2}$ ). Hence, we have to use the adaptive 
algorithm to get the optimal convergence. The adaptive algorithm contains a loop: Solve $\rightarrow$ Estimate $\rightarrow$ Mark $\rightarrow$ Refine.
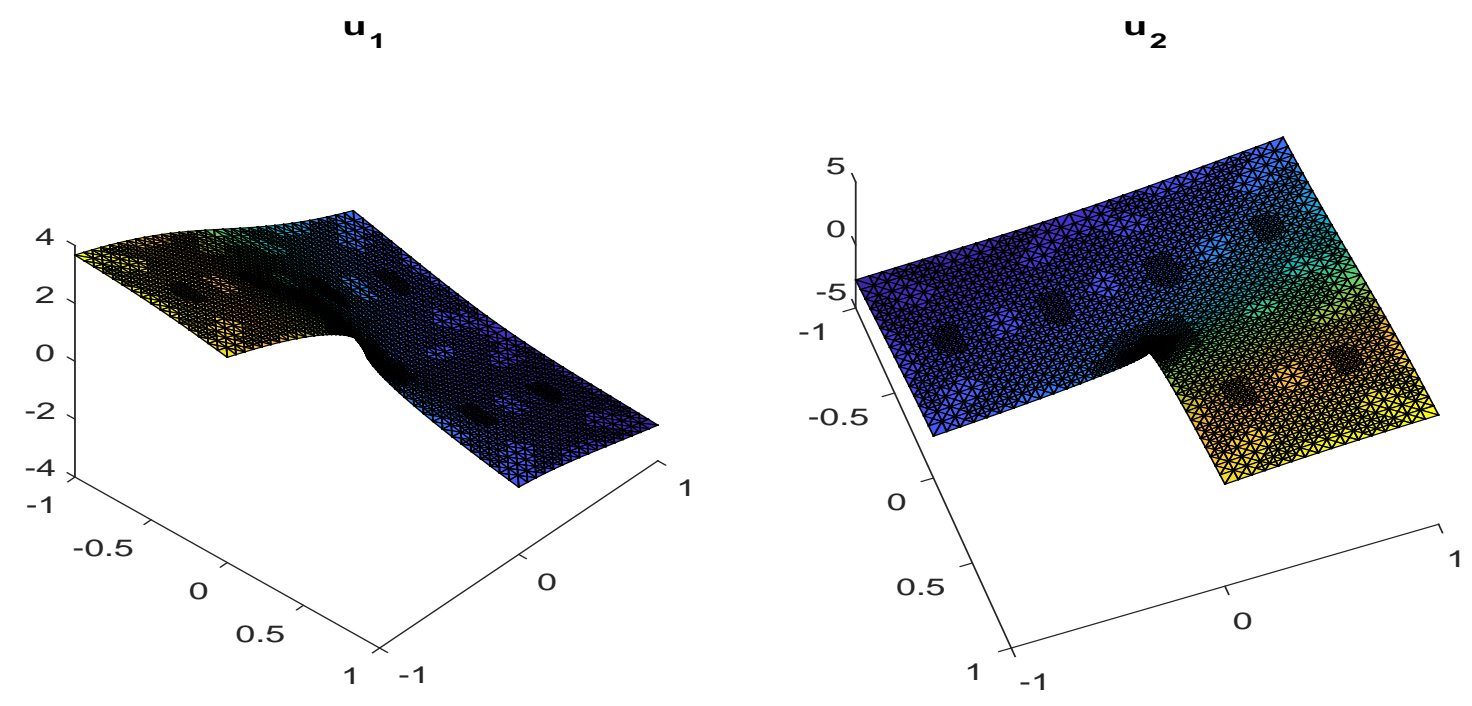

Figure 5.3. Discrete solution $\mathbf{u}_{h}=\left(u_{1}, u_{2}\right)$ of Example 5.2
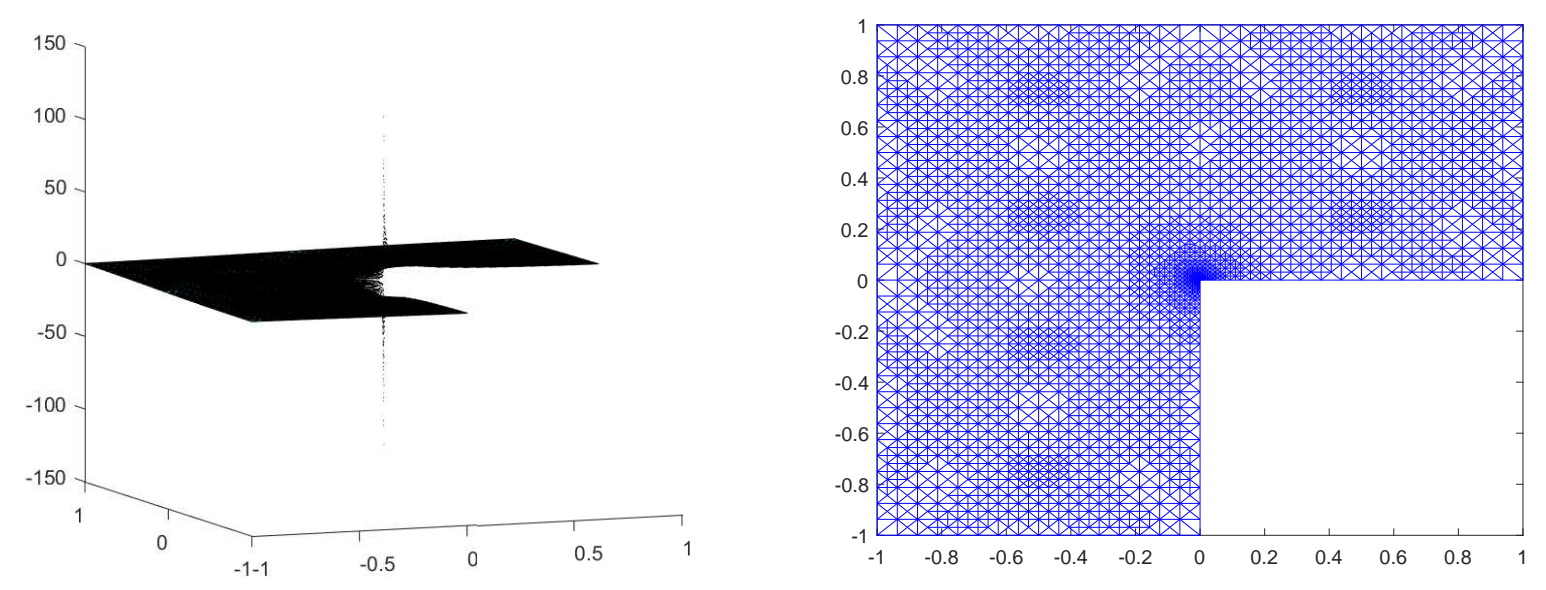

FiguRE 5.4. Discrete pressure $p_{h}$ and adaptive mesh-refinement of Example 5.2

First, we compute the discrete solutions using the primal-dual algorithm. Then, in the second step using the discrete solution we compute the error estimator $\eta$ (as defined in Theorem (4.6) ) over each element. We use the Dörlfer marking technique [14] with bulk parameter $\theta=0.3$ for the mark step and the newest vertex bisection algorithm for mesh-refinements. 
Figure 5.3 displays the discrete approximation to velocity $\mathbf{u}=\left(u_{1}, u_{2}\right)$ and the left-hand side image from Figure 5.4 show the discrete approximation to the pressure $p$. The righthand image of Figure 5.4 shows adaptive mesh generated from several iterations of adaptive refinements. In figure 5.4, due to a singularity at the origin of the state velocity and pressure variables, more mesh-refinements are observed at the origin, while other refinements are results of the adjoint variable estimator.

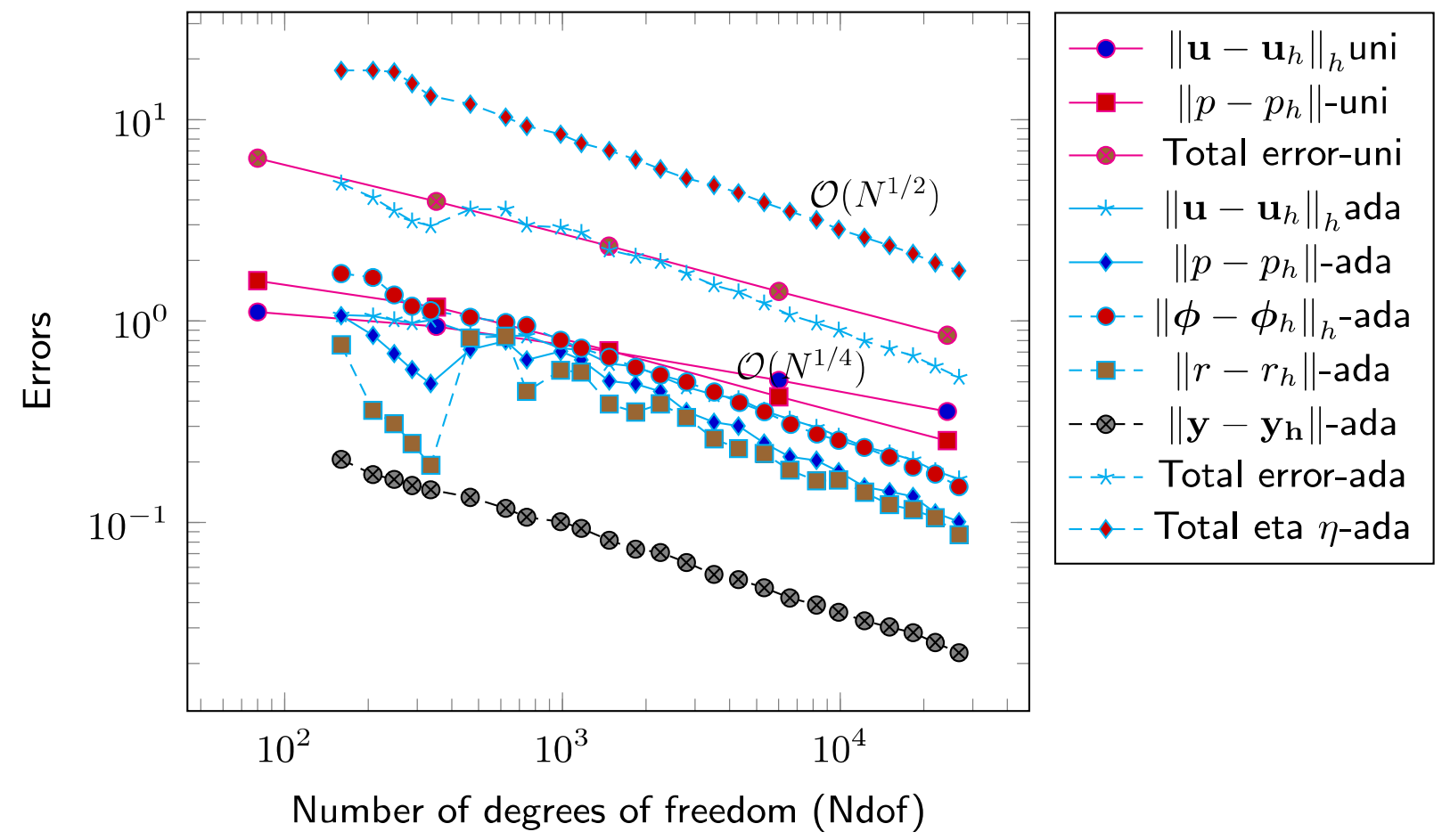

FIGURE 5.5. Convergence history with uniform and adaptive refinements for Example 5.2

Figure 5.5] depicts convergence rates for errors and estimators with uniform and adaptive refinements. The optimal convergence is achieved using the adaptive algorithm for the error in energy norm in the state and adjoint state velocity approximation, in $L^{2}$-norm of control, pressure and adjoint pressure variables. Hence, the optimal convergence for the a posteriori estimator and the total error which is the combination of all errors term as in the left-hand side of (4.9) . Here, the optimal convergence means order 0.5 with respect to Ndof.

\section{CONCLUSiOnS}

In this paper, we have developed an abstract framework for discontinuous finite element methods error analysis of both the distributed control and Neumann boundary control problems governed by the stationary Stokes equation, with control constraints. This framework 
will also work for linear elliptic and mixed optimal control problems with control constraints. The abstract analysis provides the best approximation results, which will be useful in the convergence analysis of adaptive methods and delivers a reliable and efficient a posteriori error estimators. Numerical experiments illustrate the theoretical findings. The results in the article will not directly cover the analysis of nonlinear mixed elliptic optimal control problems; however, they will be useful to analyze the nonlinear problems.

\section{ACKNOWLEDGMENT}

The first author gratefully acknowledges financial support from the National Board for Higher Mathematics (NBHM), Government of India.

\section{REFERENCES}

[1] T. Apel, P. Johannes and A. Rösch. Finite element error estimates for Neumann boundary control problems on graded meshes. Comput. Optim. Appl. 52:3-28, 2012.

[2] T. Apel, D. Sirch and G. Winkler, Error estimates for control constrained optimal control problems: discretization with anisotropic finite element meshes. Preprint SPP1253-02-06, DFG Priority Program 1253, Erlangen. 2008.

[3] T. Apel and G. Winkler Optimal control under reduced regularity. Appl. Numer. Math., 59: 2050-2064, 2009.

[4] S. Badia, R. Codina, T. Gudi and J. Gumaan. Error Analysis of Discontinuous Galerkin Methods for Stokes Problem Under Minimal Regularity. IMA J. Numer. Anal., 34:800-819, 2014.

[5] P. Bochev and M. Gunzburger. Least-squares finite-element methods for optimization and control problems for the Stokes equations. Comput. Math. Appl., 48:1035-1057, 2004.

[6] S.C. Brenner. Poincaré-Friedrichs inequalities for piecewise $H^{1}$ functions, SIAM J. Numer. Anal. 41:306-324, 2003.

[7] S.C. Brenner and L.R. Scott. The mathematical theory of finite element methods (Third edition). Springer-Verlag, New York, 2008.

[8] C. Carstensen, M. Eigel, R.H.W. Hoppe and C. Löbhard. A review of unified a posteriori finite element error control, Numer. Math. Theory Methods Appl., 5:509-558, 2012.

[9] C. Carstensen, T. Gudi and M. Jensen. A unifying theorey of a posteriori error control for discontinuous Galerkin FEM. Numer. Math., 112:363-379, 2009.

[10] E. Casas and J. P. Raymond. Error estimates for the numerical approximation of dirichlet boundary control for semilinear elliptic equations. SIAM J. Control Optim., 45:1586-1611, 2006.

[11] S. Chowdhury , T. Gudi and A.K. Nandakumaran. A framework for the error analysis of discontinuous finite element methods for elliptic optimal control problems and applications to $C^{0}$ IP methods., Numer. Funct. Anal. Optim., 36:1388-1419, 2015.

[12] P. G. Ciarlet. The Finite Element Method for Elliptic Problems. North-Holland, Amsterdam, 1978.

[13] K. Deckelnick, A. Günther, and M. Hinze. Finite element approximation of Dirichlet boundary control for elliptic PDEs on two and three dimensional curved domains. SIAM J. Numer. Anal., 48:2798-2819, 2009.

[14] W. Dörfler. A convergent adaptive algorithm for Poisson's equation, SIAM J. Numer. Anal., 33:11061124, 1996.

[15] D. A. Di Pietro and A. Ern. The mathematical aspects of discontinuous Galerkin methods. SpringerVerlag, Berlin, 2012.

[16] A. Ern and J-L. Guermond. Theory and practice of finite elements. Springer-Verlag, New York, 2004. 
[17] R. S. Falk. Approximation of a class of optimal control problems with order of convergence estimates. J. Math. Anal. Appl., 44:28-47, 1973.

[18] T. Geveci. On the approximation of the solution of an optimal control problem governed by an elliptic equation. RAIRO Anal. Numér., 4:313-328, 1979.

[19] V. Girault and P.-A. Raviart. Finite Element Approximation of the Navier-Stokes Equations. Lecture Notes in Mathematics, vol. 749, Springer, Berlin, 1979.

[20] T. Gudi. A new error analysis for discontinuous finite element methods for linear elliptic problems. Math. Comp., 79:2169-2189, 2010.

[21] T. Gudi, N. Nataraj and K. Porwal. An interior penalty method for distributed optimal control problems governed by the biharmonic operator. Comput. Math. Appl., 68:2205-2221, 2014.

[22] M.D. Gunzburger, L.S. Hou and T. Swobodny. Analysis and finite element approximation of optimal control problems for the stationary Navier-Stokes equations with Dirichlet controls. Math. Model. Numer. Anal., 25:711-748, 1991.

[23] M. Hintermüller, R.H.W. Hoppe, Y. Iliash and M. Kiewag. An a posteriori error analysis of adaptive finite element methods for distributed elliptic control problems with control constraints. ESAIM Control Optim. Calc. Var., 14:540-560, 2008.

[24] M. Hintermüller, K. Ito and K. Kunish. The primal-dual active set strategy as a semismooth Newton method. SIAM J. Optim., 13:865-888, 2003.

[25] M. Hinze. A variational discretization concept in control constrained optimization: The linear-quadratic case. Comput. Optim. Appl., 30:45-61, 2005.

[26] P. Houston, D. Schautau and T.P. Wihler. Energy norm a posteriori error estimation for mixed discontinuous Galerkin approximations of the Stokes problem. J. Sci. Comput., 22/23:347-370, 2005.

[27] D. Leykekhman and M. Heinkenschloss. Local error analysis of discontinuous Galerkin methods for advection-dominated elliptic linear-quadratic optimal control problems. SIAM J. Numer. Anal., 4:20122038, 2012.

[28] S. May, R. Rannacher and B. Vexler. Error analysis for a finite element approximation of elliptic dirichlet boundary control problems. SIAM J. Control Optim., 51:2585-2611, 2013.

[29] C. Meyer and A. Rósch. Superconvergence properties of optimal control problems. SIAM J. Control and Optimization, 43:970-985, 2004.

[30] J.-C. Nédélec. A new family of mixed finite elements in R3. Numer. Math., 50, 57-81, 1986.

[31] S. Nicaise and D. Sirch. Optimal control of the Stokes equations: conforming and non-conforming finite element methods under reduced regularity. Comput Optim Appl 49:567-600, 2011.

[32] G. Of, T. X. Phan and O. Steinbach. An energy space finite element approach for elliptic Dirichlet boundary control problems. Numer. Math., 129:723-748, 2015.

[33] A. Rösch and Boris Vexler. Optimal Control of the Stokes equations : a priori error analysis for the finite element Discretization with Postprocessing. SIAM J. Numer. Anal., 5:1903-1920, 2006.

[34] F. Tröltzsch. Optimal control of partial differential equations: Theory, methods and applications. American Mathematical Society, Providence, RI, 2010.

[35] R. Verfürth. A Review of A Posteriori Error Estimation and Adaptive Mesh-Refinement Techniques. Wiley-Teubner, Chichester, 1995.

Department of Mathematics, Indian Institute of Science, Bangalore - 560012, India

Email address: ashadond@iisc.ac.in

Department of Mathematics, Indian Institute of Science, Bangalore - 560012, India

Email address: gudi@iisc.ac.in

Department of Mathematics, Indian Institute of Science, Bangalore - 560012, India

Email address: rameshsau@iisc.ac.in 